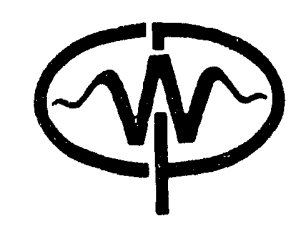

\title{
A Klein-Gordon Acoustic Theory
}

Phil D. Anno

- Thesis for Doctor of Philosophy (Geophysics)

Rcoivad by 017

JAN 291993

Center for Wave Phenomena

Colorado School of Mines

Golden, Colorado 80401

(303) 273-3557 


\section{ABSTRACT}

I propose the Klein-Gordon differential operator, from electromagnetics, as a basis for acoustic wave studies in variable-density media. A change of the dependent variable in the acoustic variable-density wave equation reveals it to be essentially the simpler Klein-Gordon equation, in disguise. Because it may be analyzed for constant-coefficient cases, the Klein-Gordon differential equation affords an attractive analytical setting for investigating the behavior of waves and rays.

Moreover, the Klein-Gordon operator is dispersive, exhibiting in the acoustic context a dispersion coefficient related to the density gradient. This means that large density gradients delay the traveltimes of waves through dispersion. Specifically, those gradients enter into a dispersion relation and phase and group velocity expressions, derived by Fourier analysis on the Klein-Gordon operator.

A companion ray-theoretical analysis of the Klein-Gordon differential equation complements one's understanding of this phenomenon. Density gradients impact ray traveltimes (appear in a new eikonal equation) when the wavelength of density variation is similar to the seismic wavelength. This eikonal leads to a general Snell's Law for acoustics, also involving density gradients. That is, those gradients contribute to ray bending at interfaces.

Many of these new results depend on the assumptions of a slowly-varying dispersion coefficient and speed. One must, however, make this same supposition about density and speed in order to obtain the classical eikonal equation and Snell's Law, as well as the familiar wave-equation dispersion relation for constant-density media. Hence, these new results are extensions of the old-addressing the rapid density variation that may accompany slow changes, over space, in the dispersion coefficient. These new relations may thus be applied for those special geological environments where speed varies slowly, yet density varies rapidly. I give interbedded salt/anhydrite sections as one important example where density undergoes abrupt changes, unaccompanied by speed changes.

The new dependent variable $q$ is related to the original pressure $p$ of the variabledensity wave equation through $p=\sqrt{\rho} q$, where $\rho$ represents density of the medium. It is the quantity $q$ that satisfies the Klein-Gordon equation. We see that $p$ is related to $q$ through an amplitude factor alone, so that variable-density traveltimes are governed by the Klein-Gordon dispersion relation.

Importantly, however, I find that the effective wave dispersion produced by discontinuities in density parameters far outweighs that due to continuous density variation. The Klein-Gordon relation describes the latter type of dispersion. Conditions for scattering at an interface impart the former type. This means that simple extensions of our typical dispersion-relation based algorithms for modeling and imaging-from the constant-density relation to the Klein-Gordon relation-will not account pro -ily for 
time delays imposed by rapid, discontinuous density variation.

The comparison of a second, variable-density differential operator with the standard operator runs as a parallel topic throughout this paper. The Klein-Gordon operator subsumes both, providing an analytical framework within which to evaluate their respective properties. It seems that, thinking of a background medium in static equilibrium-yet modeling with acoustic equations that imply dynamic equilibrium - we create density profiles for which both operators are flawed. This paradox arises because we neglect the force of gravity in the equations of motion.

To be specific, the non-standard operator is suited for rapid, continuous density variation, but precludes the possibility of jumps in density at interfaces. In contrast, one may only expect the standard operator to model, accurately, the wave response to slowlyvarying density profiles. In return for this limitation one gains the legitimate freedom to consider discontinuities in density in an acoustic medium. 


\section{TABLE OF CONTENTS}

ABSTRACT $\ldots \ldots \ldots \ldots \ldots \ldots \ldots \ldots \ldots$

ACKNOWLEDGMENTS ................... iv

Chapter 1 INTRODUCTION .................. 1

1.1 Preliminary examples . . . . . . . . . . . . . . . . . . . . 1

1.2 Problem $/$ solution overview . . . . . . . . . . . . . . 4

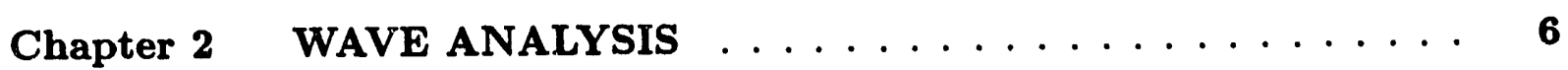

2.1 Klein-Gordon form . . . . . . . . . . . . . . . . . . . . 6

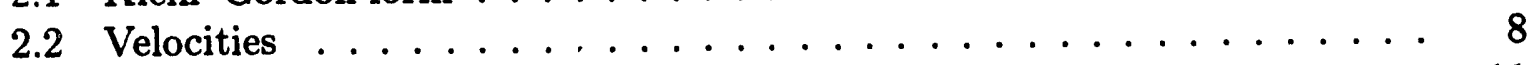

2.3 Constant-dispersion density profiles . . . . . . . . . . . . 11

Chapter 3 CONSTANT-DISPERSION MODELING AND IMAGING 15

3.1 Profiles with discontinuous density . . . . . . . . . . . . . 15

3.2 Profiles with discontinuous fractional density . . . . . . . . . 22

Chapter 4 CAUSALITY AND ENERGY CONSERVATION . . . . 24

4.1 Causal solutions . . . . . . . . . . . . . . . . . . . . . . . 24

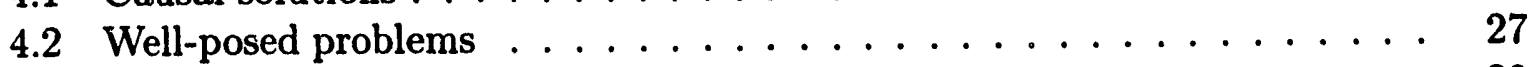

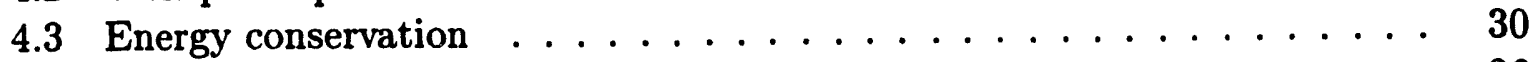

4.4 The abbreviated variable-density operator . . . . . . . . . . 36

Chapter 5 A SCALED KLEIN-GORDON WAVE EQUATION . . 39

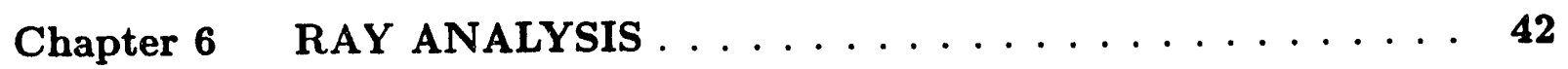

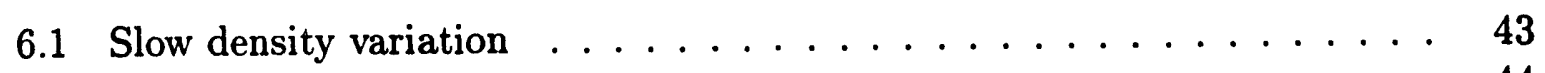

6.2 Rapid density variation . . . . . . . . . . . . . . . . 44

Chapter $7 \quad$ SCATTERING AT AN INTERFACE . . . . . . . . 46

7.1 Interface conditions . . . . . . . . . . . . . . . . 46

7.2 Plane-wave scattering . . . . . . . . . . . . . . . . . 50

7.3 Ray scattering . . . . . . . . . . . . . . . . 54

Chapter 8 VARIABLE-DENSITY OPERATOR COMPARISONS . . 61 


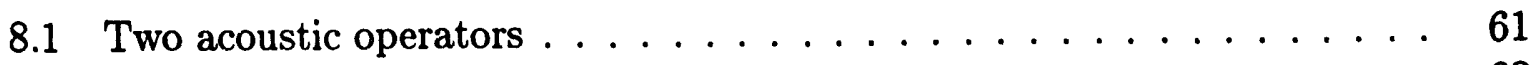

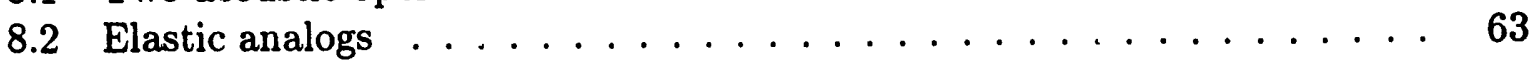

Chapter 9 FUTURE WORK $\ldots \ldots \ldots \ldots$

Chapter 10 CONCLUSIONS ................... 66

REFERENCES ........................... 69

Appendix A VARIABLE-DENSITY WAVE EQUATION . . . . . . . 71

A.1 Exact equations . . . . . . . . . . . . . . . . . . . . 71

A.2 Linear equations . . . . . . . . . . . . . . . . . . . 72

A.3 Velocity potential . . . . . . . . . . . . . 75

Appendix B AN ANALYTIC KLEIN-GORDON SOLUTION . . . 77

Appendix C ACOUSTIC-ELASTIC ANALOGS . . . . . . . . . 79

C.1 Exact elastic equations . . . . . . . . . . . . . . . . . . . 80

C.2 Linear elastic equations . . . . . . . . . . . . . . . . . 81

C.3 Acoustic equivalents . . . . . . . . . . . . . . . 82 


\section{ACKNOWLEDGMENTS}

This research was supported by the Consortium Project of the Center for Wave Phenomena, the Center for Geoscience Computing (both at the Colorado School of Mines) and, in addition, the Shell Foundation. Further support was given by the United States Department of Energy, grant number DE-FG02-89ER.14079. (This support does not constitute an endorsement by $\mathrm{DOE}$ of the views expressed in this paper.) 


\section{Chapter 1}

\section{INTRODUCTION}

In my experience, geophysicists do not associate traveltime variation with density variation in acoustic or elastic wavefield interpretation. Rather, given a constant index of refraction, density variation within the medium of propagation is associated only with amplitudes. This point of view prevails because density does not occur as a variable in classical results such as Snell's Law or the eikonal equation. Nevertheless, in this paper I predict, analytically, a continuum of density effects on acoustic wavefields-including a dispersive traveltime delay when density variation is rapid. I also examine the ability of a common imaging algorithm to cope with this time delay.

\subsection{Preliminary examples}

Figure 1.1 demonstrates such delays. The traces in the figure are computed, onedimensional reflection data. Each trace corresponds to a different density model, with each succeeding model exhibiting greater density variation.

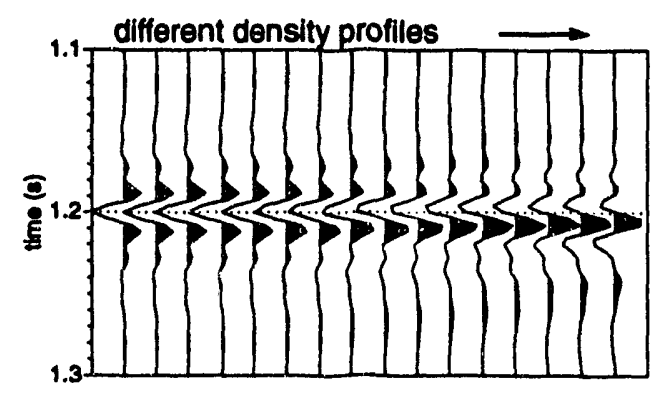

FIG. 1.1. Computed, one-dimensional reflection data for a propagating plane wave. Each successive trace corresponds to a different density model in Figure 1.2. The source and receiver are at the surface of the model for each trace. For each trace, the signal began its journey as a zero-phase wavelet. These data do not contain the effects of scattering from points of discontinuous density in Figure 1.2.

The sequence of models is depicted in Figure 1.2. The characteristic speed $c$ for each model was held constant at $3000 \mathrm{~m} / \mathrm{s}$, and the halfspace drawn in Figure 1.2 is at a depth of $1800 \mathrm{~m}$. We therefore expect a nominal reflection time of $1.2 \mathrm{~s}$. One may note, however, that the rightmost trace in Figure 1.1 shows an apparent time delay. The 
main lobe of energy reflected from the halfspace is a "peak" - rather than a "trough"at $1.208 \mathrm{~s}$. Since the polarity of recorded data is often unknown in seismic applications, the unsuspecting seismic interpreter would calculate the depth to the halfspace as about $1812 \mathrm{~m}$, a $12 \mathrm{~m}$ error.

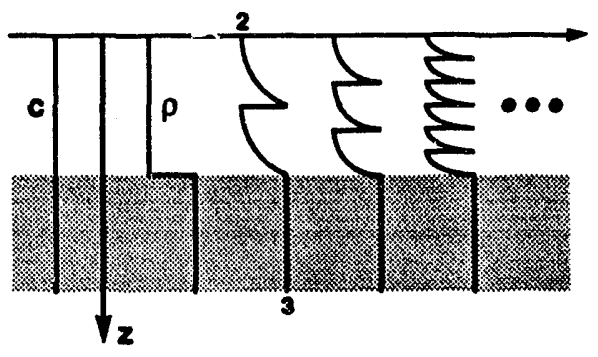

FIG. 1.2. A suite of density models, for analyzing how the rate of density variation affects wave propagation. The speed $c$ is held constant. With the exception of the first model, density $\rho$ varies exponentially between 2 and $3 \mathrm{~g} / \mathrm{cm}^{3}$ above a halfspace.

I will refer back to the data in Figure 1.1 with more information as the paper develops. Though, depending on the application, this depth error may not be significant, we will find that the time delay on the rightmost trace of Figure 1.1 corresponds, in quantitative terms, to rather mild density variations. Moreover, this delay increases when all the scattering effects are incorporated into the modeling calculation.

I do not wish to advertise the models in Figure 1.2 as being representative, necessarily, of typical geological environments. Rather, these models are useful for studying the influence of density on wave propagation - without the added traveltime complexity that results when speed also varies. Indeed, Figure 1.3 verifies that, in most circumstances, speed changes when deusity changes.

These velocity-density data also point out an exception to the rule for sedimentary rocks. The speeds for salt and anhydrite are similar, though their densities differ drastically. This suggests that density variation will play the dominant role in the response of seismic waves to interbedded sections of salt and anhydrite. Indeed, based on the results of this paper, I contend that successful seismic imaging of geological structures beneath such sections will depend primarily on operators that acknowledge the influence of density variation on the wave response. Large areas of the Middle East contain this type of subsurface environment. The common imaging operators currently in use in the seismic exploration industry are constant-density operators (Claerbout, 1985).

Tom Davis (personal communication) describes another geological setting where density dominates over speed. Gold deposits in South African metamorphic rocks often exhibit significant density contrasts with the surrounding host rock, though speed remains a constant $5000 \mathrm{~m} / \mathrm{s}$ throughout. 


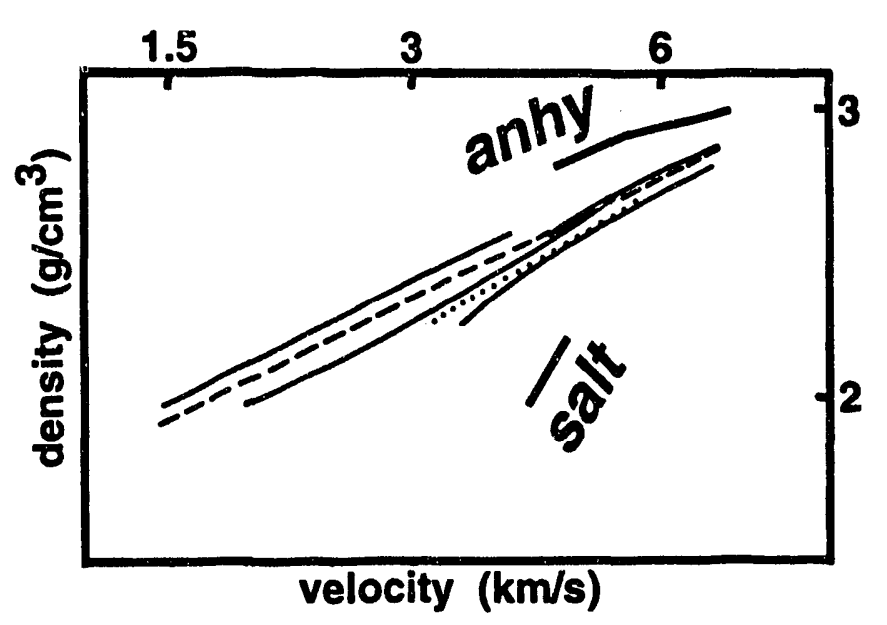

Fig. 1.3. Velocity as a function of density, for sedimentary rocks. Note that salt and anhydrite have similar velocities, but their densities differ greatly. These data were taken from Dobrin (1976).

The foregoing examples are not meant to imply, however, that density variation must dominate over speed variation in order for this study to be relevant. I think that the reader will find ii sensible to conclude from this work that density variation may induce a traveltime delay, whether or not speed varies rapidly. Nonetheless, a number of the aralytical results I obtain are couched in the framework of slow variation in speed within any single, continuous geological unit. This same underlying assumption on the speed function also underlies classical results like Snell's Law and the eikonal equation. I reveal that acoustic wave equations do not demand, for the sake of analytical tractability, the same assumption of slow variation for density. I thus extend the classical results to the case of rapid density variation.

Importantly, I will derive, exactly and asymptotically, dispersion relations which predict dispersive time delays as waves and rays propagate through media where density varies rapidly. I will also illustrate, numerically, that this type of dispersion may be interpreted in terms discussed by O'Doherty and Anstey (1971). These authors explain this phenomenon in terms of multiply-reflected signals separated by small time delays (short-period multiples). I say this, now, to emphasize that the wave process that I speak of is not a new one.

Unlike O'Doherty and Anstey's work, I have neglected for the moment to calculate multiple reflections between the density discontinuities in Figure 1.2. This does not mean that these multiples are unimportant to the wave response. Indeed, they will prove to limit fundamentally any simple extension of the constant-density modeling and imaging algorithms we employ commonly for seismic exploration applications.

The dispersion relation that describes propagation in a variable-density acoustic medium can be written in the Klein-Gordon form (see e.g., Bleistein, 1984) found in 
electromagnetic applications. Applied to the oversimplified data in Figure 1.1, this dispersion relation images successfully the halfspace in Figure 1.2. Figure 1.4 displays the migrated images.

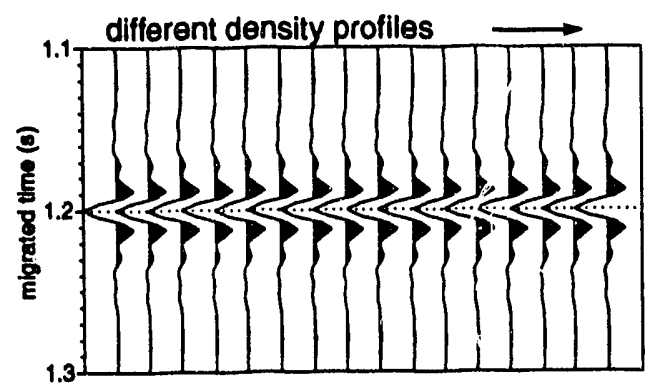

FIG. 1.4. The output of Klein-Gordon migration on the data in Figure 1.1. The halfspace in Figure 1.2 is imaged correctly.

This imaging example illustrates well the action of the Klein-Gordon dispersion relation. Those familiar with the classical (constant-density) wave-equation relation will realize that it makes no change to data like those in Figure 1.1. The classical operator does not move reflection data possessing zero horizontal wavenumber.

\subsection{Problem/solution overview}

The acoustic initial value problem

$$
\begin{gathered}
\rho(x) \nabla \cdot\left[\frac{\nabla p}{\rho}\right]-\nabla \cdot\left[\frac{\nabla \rho}{\rho}\right] p-\frac{1}{c^{2}(x)} \frac{\partial^{2} p}{\partial t^{2}}=-f(x, t), \\
p(x, 0)=r(x), \quad \frac{\partial}{\partial t} p(x, 0)=s(x),
\end{gathered}
$$

proves fruitful for exploring propagation in a variable-density medium. This governing partial differential equation, referred to as the variable-density wave equation, is derived in detail in Appendi: $\wedge$ as equation (A-19). The symbols $p, \rho, c$, and $f$ denote a small pressure fluctuation, density of the acoustic medium, characteristic speed, and an acoustic source, respectively. Equations (1.2) provide initial data on pressure and its derivative at time $t=0$.

Oftentimes, the fractional density term (where $\nabla \rho / \rho$ is fractional density) in equation (1.1) is neglected (Claerbout, 1985; Wapenaar and Berkhout, 1989). DeSanto (1992) gives a derivation which preserves it. I show in this paper that, without that term, equation (1.1) does not conserve energy, unconditionally, unless the fractional density of a medium is zero everywhere. Practically, that vanishing may be posited with sound physical justification for bona fide acoustic applications. 
On the other hand, much credence is given to modeling and imaging theories (Claerbout, 1985) which treat the solid Earth according to acoustic wave equations. At a minimum, these theories must correctly predict seismic traveltimes to be judged successful. Clearly, fractional density can be large in solids. Furthermore, based on this paper, that fractional density term can effect a traveltime delay when density varies rapidly. Hence, I include the term in my analysis. I will, however, at each "ppropriate juncture point out how its exclusion from equation (1.1) affects results. Essentially, none of the new concepts presented hinge on its presence.

It is also important to realize that both of the variable-density equations that we will consider spring from an acoustic medium that is in dynamic-not static-equilibrium, before waves are introduced. This results from neglecting such forces as gravity in the equations of motion. We will find this initial state to be of practical importance, because it restricts the density fun :tions for which these differential operators produce accurate results.

We shall first undertake a limited, but exact, analysis of equation (1.1), accompanied by initial conditions. This reveals that waves do not propagate with speed $c$, as they do in constant-density media. Instead, speed of propagation is wavelength dependent. That is to say, a variable-density acoustic medium is dispersive, though assumed to be perfectly elastic. The coefficient responsible for this dispersion is proportional to the gradient and curvature of the density function. Consequently, rapid density variations produce a dispersive traveltime distortion.

Following that exact analysis, I perform an asymptotic analysis beginning with equation (1.i). This approach extends the scope of speed functions $c$ that can be considered analytically, though at the expense of making the usual high frequency wavefield approximation (Cerveny et al., 1977). I selectively scale the Klein-Gordon differential equation to obtain a ray analog to the wave analysis. Importantly, the scaled equation admits asymptotics not only for slow variation in $\rho$ and $c$, the usual limitation, but also allows rapid variation in $\rho$. A hierarchy of asymptotic dispersion relations emerges for the variable-density wave equation. That hierarchy embeds previous results for slow density variation as a special case, and points again to the Klein-Gordon dispersion relation. 


\section{Chapter 2}

\section{WAVE ANALYSIS}

We begin our investigation by analyzing equation (1.1) for the case of constant $c$ and specifically restricted density functions as defined below. Though these are special cases, they can be solved exactly - at least as far as is needed to identify several important propagation characteristics in a variable-density medium. Furthermore, the restrictions of this exact analysis can be met locally in any inhomogeneous medium. Consequently, one would expect that wave phenomena predicted for these special cases will in some sense manifest themselves in the case of general inhomogeneity.

The main aim of the wave analysis will be to derive a dispersion relation and phase and group velocity expressions for particular constant-c, variable- $\rho$ media. These expressions will establish that density gradients act as a dispersing mechanism in acoustic media, causing dispersive wave propagation.

The key step of analysis is a change of the devendent variable from pressure $p$ to $q$ where

$$
q \stackrel{\text { def }}{=} \frac{p}{\sqrt{\rho}} .
$$

This change transforms equation (1.1) to the well-studied Klein-Gordon form, giving the advantage of a much simpler and well-trod path. For our constant- $c$ variable- $\rho$ example, Fourier transforms reduce the new equation on $q$ to an algebraic expression; the solution for $q$ is expressed in terms of inverse Fourier transforms. One deduces phase and group velocity after locating the singularities in the integrand of the transforms.

\subsection{Klein-Gordon form}

The Klein-Gordon differential form results from substitution of the change of variable of equation (2.1) into equation (1.1), giving

$$
\Delta q-\frac{b^{2}}{c^{2}} q-\frac{1}{c^{2}} \frac{\partial^{2} q}{\partial t^{2}}=-\frac{1}{\sqrt{\rho}} f(x, t),
$$

with a dispersion coefficient given by

$$
b^{2} \stackrel{\text { def }}{=} c^{2} \frac{\Delta \sqrt{\rho}}{\sqrt{\rho}} .
$$


The symbol $\Delta$ designates the Laplacian operator. The identity

$$
\frac{\Delta \sqrt{\rho}}{\sqrt{\rho}} \equiv \frac{\Delta \rho}{2 \rho}-\frac{\nabla \rho}{2 \rho} \cdot \frac{\nabla \rho}{2 \rho}
$$

is central to collapsing terms to a single Laplacian on $\sqrt{\rho}$.

The Klein-Gordon differential equation also governs electrornagnetic waves in the ionosphere (Jackson, 1962) and de Broglie waves for free relativistic particles. In the electromagnetic context, the symbol $b$ in the Klein-Gordon operator corresponds to a resonant frequency of the plasma and serves to introduce dispersion. Relative curvature $\Delta \sqrt{\rho} / \sqrt{\rho}$ of the square ront of the density function, scaled by $c^{2}$, int, oduces dispersive wave behavior in the present context. Equation (2.4) expresses the relative curvature of $\sqrt{\rho}$ as a weighteci sum of curvature and fractional density terms in $\rho$.

We see from equation $(2.1)$ that the Klein-Gordon field is related to a variabledensity field through an amplitude factor alone, albeit a spatially varying factor. This means that variable-density traveltimes : $:$ e governed totally by the Klein-Gordon dispersion relation (that I shall derive kelow). Equation (2.î) does not, however, appear to apply - at least unconditionally - at discontinuities in either the dispersion or speed coefficients. It is at those discontinuities where physical coefficients and their derivatives are lowved to become singular. Below I derive conditions which must be enforced to keep Alact the integrity of the wave equation, and equatio. (2.1), at boundaries.

The relative curvature quantity in equation (2.3) may be re-expressed in terms of the divergence of fractional density to see that

$$
b^{2} \stackrel{\text { def }}{=} c^{2}\left[\frac{1}{2} \nabla \cdot\left(\frac{\nabla \rho}{\rho}\right)+\frac{\nabla \rho}{2 \rho} \cdot \frac{\nabla \rho}{2 \rho}\right] .
$$

This form of the dispersion coefficient indicates that the operators of equations (1.1) and (2.2) are dispersive, whether or not the divergence term - the second term in equation (1.1), the first here-is included. In physical terms, the medium is fundamentally dispersive in either case. In mathematical terms, the Klein-Gordon form of the variabledensity wave equation is attainable without the fractional density term.

Moreover, equations (2.4) and (2.5) make it clear that $b$ may be imaginary $\left(b^{2}<0\right)$ with this divergence term, but not without it. There is no cause for alarm. A negative value of $b^{2}$ corresponds physically to a negative relative curvature in the function $\sqrt{\rho}$, and is necessary to span the space of all possible density variations.

Finally, I point out that the Klein-Gordon operator is self-adjoint while the operator of equation (1.1) is not. Propagation problems for variable-density media may therefore be done in $q-$ rather than $p-$ for the sake of analytical convenience if for no other reason. 


\subsection{Velocities}

Employing the change of variable in equation (2.1), I identify

$$
\begin{gathered}
\Delta q-\frac{b^{2}}{c^{2}} q-\frac{1}{c^{2}} \frac{\partial^{2} q}{\partial t^{2}}=-\frac{1}{\sqrt{\rho}} f(x, t), \\
q(x, 0)=\frac{1}{\sqrt{\rho}} r(x), \quad \frac{\partial}{\partial t} q(x, 0)=\frac{1}{\sqrt{\rho}} s(\approx)
\end{gathered}
$$

as the equivalent acoustic initial value problem in $q$. Consider the constant-coefficient case for the Klein-Gordon operator of equations (2.6). This case limits $c$ to a constant $\left(c_{o}\right)$ and $\rho$ to that class of density functions satisfying

$$
\Delta \sqrt{\rho}=\frac{b_{o}^{2}}{c_{o}^{2}} \sqrt{\rho},
$$

with $b_{o}^{2} / c_{o}^{2}$ constant. Herein lies a chief analytical reward for the change of variable. The Klein-Goidon representation makes it possible to work with a constant-coefficient-yet variable-density - wave equation.

That is, a single value for $b_{o} / c_{o}$ implies a density function, irom equation (2.7). Hence, Klein-Gordon modeling and imaging algorithms may "think" in terms of the dispersion and speed for a layer, rather than speed and a density function. Indeed, one can assert from equations (2.6) that the canonical acoustics problem is ccinstant $b$ and constant $c$-not constant $\rho$ and constant $c$-as one might presume. Constant density is a special case described jointly by $b_{o}=0$ in the $q$ variable, and $p=\sqrt{\rho_{o}} q$.

It is important, in turn, that one understands the solutions to equation (2.7). One must ensure that the density function implied by a given value of $b_{o}$ is physical. Suffice it to say now that there are such solutions to equation (2.7) and that they may be used to construct many physically realizable density functions.

To proceed toward velocity expressions, I define the forward Fourier transfor $m$ on $q$ as

$$
Q(k, w)=\int_{-\infty}^{\infty} d x \int_{0}^{\infty} d t q(x, t) e^{-i(k \cdot x-\omega t)}
$$

and apply it to equations (2.6) to obtain

$$
\left[-k^{2}+\frac{w^{2}}{c_{o}^{2}}-\frac{b_{o}^{2}}{c_{o}^{2}}\right] Q=-\frac{1}{c_{o}^{2}} \nu(k) *\left[S(k)-i \omega R(k)+c_{o}^{2} F(k, \omega)\right],
$$

provided that

$$
\lim _{t \rightarrow \infty} q=O\left(e^{\Im(\omega) t}\right), \quad \lim _{|x| \rightarrow \infty}(q,|\nabla q|)=0 .
$$

Integral transforms of derivatives invariably entail an integration-by-parts procedure, the source of the limits that accompany equation (2.9). Though one usually acknowledges implicitly these limiting conditions on $q$, their explicit stipulation will serve us well in 
instances to come.

The scalar quantity $k$ in equation (2.9) represents the magnitude of the $k$ vector and $S, R$, and $F$ are transforms of the initial data and the source function in equation (2.6). The function $\nu$ signifies the transform of $1 / \sqrt{\rho}$,

$$
\frac{1}{\sqrt{\rho(x)}}=\frac{1}{(2 \pi)^{3}} \int_{-\infty}^{\infty} d k \nu(k) e^{i k \cdot x}
$$

The * operator signifies convolution, defined as

$$
\nu(\boldsymbol{k}) * S(\boldsymbol{k}) \stackrel{\text { def }}{=} \frac{1}{(2 \pi)^{3}} \int_{-\infty}^{\infty} d \boldsymbol{k}^{\prime} \nu\left(\boldsymbol{k}^{\prime} ; S\left(\boldsymbol{k}-\boldsymbol{k}^{\prime}\right)\right.
$$

for example. Significantly, convolutions arise via the convolution theorem (found in Bracewell (1986)) because, though $b$ and $c$ are constants, $\rho$ is not. Still, this definition of convolution differs, due to the factors of $1 / 2 \pi$, from the standard one presented by Bracewell. The definition given here is motivated by a difference, likewise, in our definitions of Fourier transforms.

The solution $q$ can now be written as an inverse Fourier transform of $Q$. We solve for $Q$ in equation (2.9), then write

$$
q(x, t)=\frac{1}{(2 \pi)^{4}} \int_{-\infty}^{\infty} d k \int_{C} d \omega\left[\nu(k) * \frac{i \omega R(k)-S(k)-c_{o}^{2} F(k, \omega)}{\omega^{2}-c_{o}^{2} k^{2}-b_{o}^{2}}\right] e^{i(k \cdot x-\omega t)} .
$$

The path of integration $(C)$ is a straight line from $-\infty$ to $\infty$ above all singularities in the $\omega$-plane. The zeros of the denominator in the integrand of equation (2.13) establish a key relation between frequency and its corresponding wavenumber in a variable-density medium. Those zeros occur at

$$
k^{2}=\frac{\omega^{2}}{c_{o}^{2}}-\frac{b_{o}^{2}}{c_{o}^{2}} \text {. }
$$

Indeed, equation (2.14) is the Klein-Gordon dispersion relation.

The data in Figure 1.1 were computed in $\left(\omega, k_{z}\right)$-space, $z$ denotin's the depth coordinate, using a phase-shift algorithm (Gazdag (1978)) that employs equation (2.14). Figure 2.1 discloses that each trace corresponds to using a different value of $b_{o}^{2}, c$ held constant. The wavelet is propagated at speed $c_{o}$ and without distortion when the dispersion coefficient is zero. This result is anticipated from equation (2.14). One gets a gencral impression from Figure 2.1 that the energy in the wavelet is delayed to larger times, the larger the dispersion coefficient. It is also apparent at the largest dispersion values that the lower frequencies propagate at a lower effective speed. Let us pursue these observations.

According to the exponent of the exponential in equation (2.13), planes of constant 


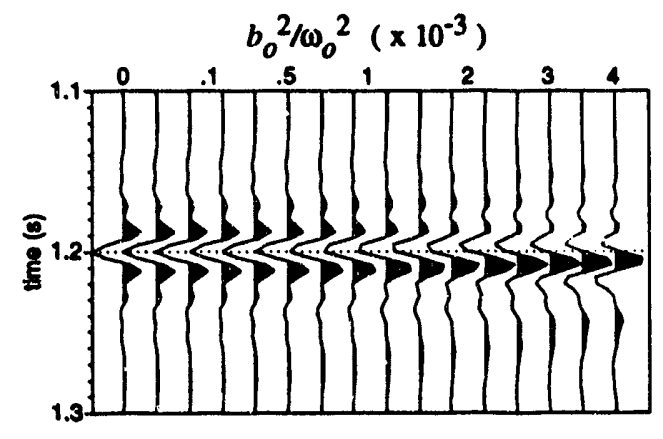

Fig. 2.1. Figure 1.1 data, labeled by their ratio of dispersion to dominant frequency, $\omega_{0}$. Equation (2.14) suggests this ratio as an appropriate dimensionless measure of dispersion. These data do not contain the effects of scattering from points of discontinuous density in Figure 1.2.

phase travel with speed $\omega / k$ to give

$$
\begin{aligned}
v_{p} & =\frac{\sqrt{c_{o}^{2} k^{2}+b_{o}^{2}}}{k} \\
& =c_{o} \sqrt{1+\frac{1}{k^{2}}\left(\frac{\Delta \sqrt{\rho}}{\sqrt{\rho}}\right)_{o}} .
\end{aligned}
$$

Group (energy) velocity $v_{g}$, given by $d \omega / d k$, is

$$
v_{g}=c_{o} / \sqrt{1+\frac{1}{k^{2}}\left(\frac{\Delta_{\sqrt{\rho}}}{\sqrt{\rho}}\right)_{o}}
$$

from equation (2.14). Clearly, inhomogeneity in density imposes a dispersive traveltime distortion on propagating acoustic waves. Equations (2.15) and (2.16) disclose that the diagnostic propagation speeds depend on the relative curvature of $\sqrt{\rho}$ as well as wavenumber, and $v_{p} \neq c_{o} \neq v_{g}$. These velocities tend to the same value $c_{o}$ only for very large $k$. This point, related to the bandwidth of data, will re-emerge when we obtain an analytic representation for the data in Figure 2.1.

It should be noted that, when the relative curvature of $\sqrt{\rho}$ is large and negative, $v_{g}>c_{o}$ from equation (2.16). Jackson (1962) cites an analogous class of electromagnetic media and dispells convincingly the myth that this inequality cannot occur without violating the notion of causality. Gioup velocity is no longer a meaningful physical concept in this case, nor is it the energy velocity. The concept of a group velocity $d \omega / d k$ 
arises through approximation of the relation between $\omega$ and $k$,

$$
\omega(k)=\omega\left(\dot{\kappa}_{o}\right)+\left.\frac{d \omega}{d k}\right|_{k_{o}}\left(k-k_{o}\right)+\ldots
$$

The quantity $d \omega / d k$ is thus seen to have special significance when $\omega$ is a weak (linear) function of $k$, not so when a medium is highly dispersive. The speed of energy propagation through a highly dispersive medium is much more difficult to characterize.

\subsection{Constant-dispersion density profiles}

The above dispersion relation and velocities are contingent upon constant coefficients in equation (2.6). That is, speed $c$ must be constant and $\sqrt{\rho}$ must satisfy a differential equation, namely equation (2.7). In this section I present several of the underlying density solutions for three-dimensional and one-dimensional (stratified) media. Any linear combination of these density functions is valid because equation (2.7) is linear and homogeneous in $\sqrt{\rho}$. Figure 2.2 graphs, in terms of $\sqrt{\rho}$, several of the functions which satisfy equation (2.7) for layered media.

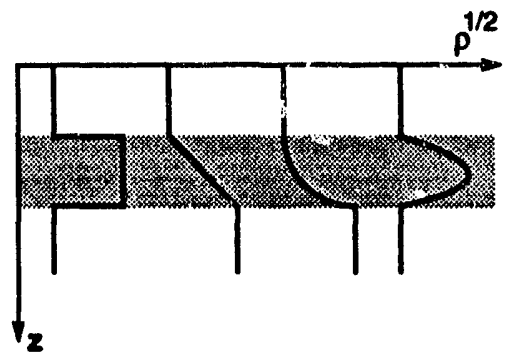

FIG. 2.2. Constant-dispersion density forms in stratified media. These functions are-from left to right - constant, inear, exponential, and sinusoidal in $\sqrt{\rho}$.

It will be obvious that these density solutions are not physical over an unlimited domain of their independent space variable. For example, the densities implied by fixing $b_{o} / c_{o}$ may become much too small (even negative) beyond a certain region in space. Consequently, the spatial domain over which speed and dispersion can be fixed "legitimately" must be restricted, to restrict density to physical values. This constraint is important for applications which require legitimate handling of transmission effects on amplitude. The necessary transmission and reflection coefficients are derived in a section on scattering at interfaces.

Consider a density' scatterer having three-dimensional spherical symmetry. Radial distance $r$ defines the distance from the center of the scatterer. For this symmetry, 
equation (2.7) assumes the form of a modified Bessel differential equation (Abramowitz and Stegun, 1965) with solutions

$$
\sqrt{\rho}= \begin{cases}C_{1} \frac{\sinh (|\kappa| r)}{|\kappa| r} & , \kappa \stackrel{\text { def }}{=} \frac{b_{o}}{c_{o}}, b_{o}^{2}>0 \\ C_{2} \frac{\cosh (|\kappa| r)}{|\kappa| r} & , C_{1}, C_{2} \text { arbitrary constants. }\end{cases}
$$

These two solutions may be combined linearly to produce density functions that decay or grow with $r$. The choice one makes for the ratio $\left|b_{o}\right| / c_{o}$ controls the rate of growth or decline away from the center of the scatterer. Equations (2.17) offer three-dimensional profiles for studying the effect of density scatterers on wavefield dispersion-independent of wave phenomena that originate from variable $c$.

Let us now turn to density solutions for stratified media, taking the depth coordinate to be $z$. Equation (2.7) simplifies greatly for $\rho=\rho(z)$. By elementary techniques one finds solutions

$$
\sqrt{\rho}= \begin{cases}C_{1} e^{\kappa z}, C_{2} e^{-\kappa z} & , b_{o}^{2}>0 ; \\ C_{1} \cos (|\kappa| z), C_{2} \sin (|\kappa| z) & , b_{o}^{2}<0 .\end{cases}
$$

For real $b_{o}$ the ratio $\left|b_{o}\right| / c_{o}$ again sets a rate of growth or decay for the density function. For imaginary $b_{o},\left|b_{o}\right| / c_{c}$ determines a wavenumber for sinusoidal density oscillation. Equations (2.18) provide suitable density functions for analyzing the effects of layered density gradients on acoustic wave propagation.

It is also of interest to consider those density functions given by a constant fractional density $\nabla p / \rho$. Though this condition restricts density more than equation (2.7), one discovers (by expanding the leftmost term of equation (1.1)) that this offers the sole example where equation (1.1) yields to analysis by Fourier transforms. If one chooses

$$
\frac{\nabla \rho}{\rho}= \pm 2 \kappa \hat{\gamma}_{o}, \quad \hat{\gamma}_{o} \stackrel{\text { def }}{=} \frac{\nabla \rho}{|\nabla \rho|}
$$

then

$$
\frac{\Delta \sqrt{\rho}}{\sqrt{\rho}}=\kappa^{2}
$$

as before. That is to say, forcing fractional density to be constant forces the same on the relative curvature of $\sqrt{\rho}$. For the case of equations (2.19)

$$
\rho(x)=C e^{ \pm 2 \kappa \hat{\gamma}_{0} \cdot x}
$$

the same as the exponential solutions in equations (2.18).

As noted above, the density solutions presented here do not take on physical values over the entire domain of their independent variable. I shall address this difficulty by giving formulas that calculate a maximum range $\delta z$ over which the depth coordinate of equations (2.18) may vary for one constant-coefficient subsurface layer. 
Let us first derive a range formula for the real, exponential solutions of equations (2.18). Write

$$
\rho(\delta z)=\rho_{\min } e^{2 \kappa \delta z},
$$

where $\rho_{\min }$ is the smallest value we wish to accept for density. One then solves for that $\delta z$ such that

$$
\rho_{\min } e^{2 \kappa \delta z}=\rho_{\max },
$$

obtaining

$$
\delta z=\frac{1}{2} \frac{c_{o}}{\left|b_{o}\right|} \ln \left(\frac{\rho_{\max }}{\rho_{\min }}\right) .
$$

Equation (2.21) gives the restriction on $\delta z$ necessary to keep density values between $\rho_{\min }$ and $\rho_{\max }$, based on the ratio of speed and the dispersion coefficient in a layer.

One can follow similar logic to derive a range formula for the sinusoidal solutions of equations (2.18). For those solutions I obtain

$$
\delta z=\frac{c_{o}}{\left|b_{o}\right|}\left(\pi-2 \arcsin \sqrt{\frac{\rho_{\min }}{\rho_{\max }}}\right) .
$$

The value of $\rho_{\max }$ specifies the peak of the sinusoid.

Figure 2.3, computed from equations (2.21) and (2.22), presents a catholic view of the restriction on subsurface layer thickness-imposed by holding $b_{o} / c_{o}$ constant. First, we see that dispersion may be assumed constant over greater depth intervals for sinusoidal density variation than for exponential variation. Also, the larger the dispersion ccitificient that one specifies, the narrower the subsurface interval over which that value cas be sustained, sill maintaining density within pre-established bounds. In physical terms, each value of $b_{o}$ implies a maximum distance to an interface in the medium of propagation. Finally, as one might surmise, broadened upper or lower bounds on density justify fixing the dispersion coefficient over a greater depth range.

Given the range re riction dictated by finite $b_{o} / c_{o}$, let us examine what happens when $b \equiv 0$ in equation (2.6). When $b \equiv 0$ the Klein-Gordon equation reduces to the constant-density form of the wave equation. To my surprise, this special restriction on $b$ does not also impose constant density. This means that-in the $q$ variable-variabledensity modeling can be accomplished with the constant-density wave equation. This statement is true for all density functions that satisfy Laplace's equation,

$$
\Delta \sqrt{\rho}=0 .
$$

These functions $\sqrt{\rho}$ do not cause wavefield dispersion.

Of course, many functions satisfy equation (2.23), particularly when $\sqrt{\rho}$ varies over two spatial dimensions. In two dimensions, the real and imaginary parts of all analytic functions are available as variable-density profiles for a constant-density form of modeling. The required density functions are quadratic in $z$ for $\rho=\rho(z)$. 


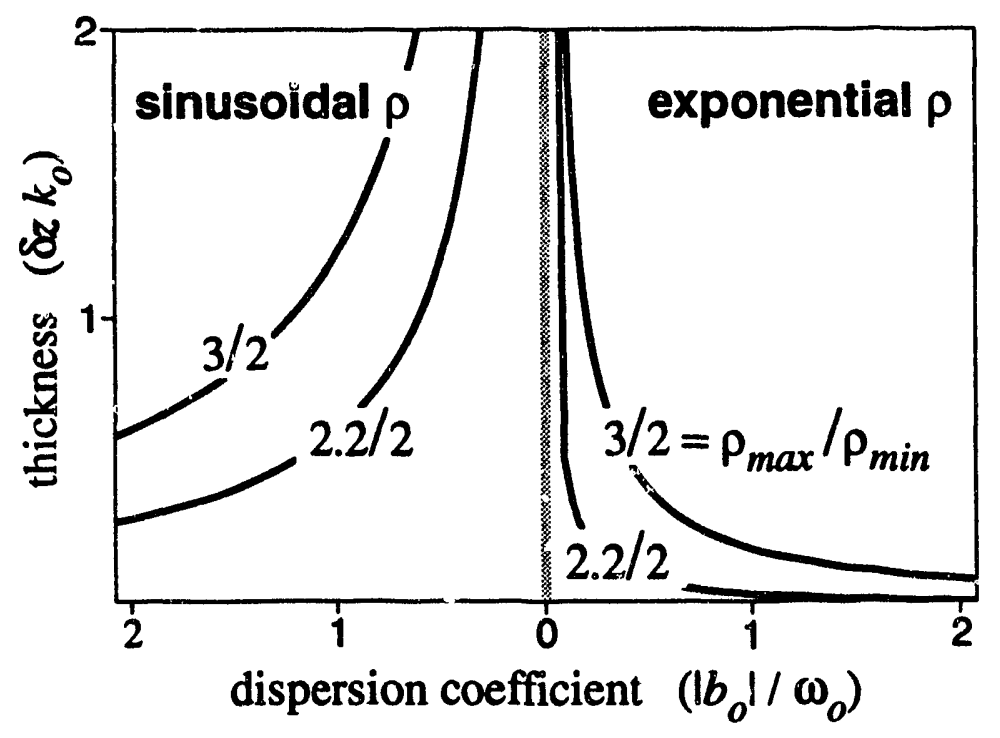

Fig. 2.3. Maximum-allowable subsurface layer-thickness, when the dispersion coefficient is held constant. Density takes on unphysical values if this thickness is exceeded. The right side shows thicknesses calculated for exponential density functions, the left for sinusoidal functions. Those thicknesses and the dispersion are normalized against a reference wavenumber $k_{o}$ and frequency $\omega_{o}$, respectively. A density ratio $\rho_{\max } / \rho_{\min }$ parameterizes assigned bounds on density. 


\section{Chapter 3}

\section{CONSTANT-DISPERSION MODELING AND IMAGING}

In this chapter we will explore, numerically, wave propagation for several classes of density models. These models are based on the constant-dispersion, prototype density functions derived above. One aim will be to demonstrate equivalency between KleinGordon solutions, $q$, and variable-density solutions, $p$, after scaling $p$ by the square root of density. These data comparisons illustrate, furthermore, the physical interpretation of the dispersion coefficient. We will come to view this coefficient as a term that incorporates the dispersion due to multiply-reflected energy from continuous density variation.

I also employ a simple extension of phase-shift migration (Gazdag, 1978) - using the Klein-Gordon dispersion relation. The goal is to image the halfspace of Figure 1.2, in spite of the data dispersion due to density variation. After all, the dispersion coefficient required for a Klein-Gordon migration could be calculated directly from density measurements that are commonly taken in wells. The synthetic imaging tests I discuss here show that simple extensions of our common imaging algorithms will not work. These algorithms cannot account for dispersion from discontinuities, in all likelihood the dominant source of dispersion in a typical density profile.

\subsection{Profiles with discontinuous density}

Figure 3.1 helps one pictur the two distinct philosophies used to compute the $p$ and $q$ fields displayed in this chapter. The $p$ solutions were computed by discretizing the density model into a large number of constant-density subdivisions, then employing a constant-density solution, $e^{i \omega \tau}$, to propagate waves between interfaces. In thli case, equation (7.33) describes accurately the scattering process at interfaces between these subdivisions. Claerbout (1976) describes an algorithm which incorporates all resulting multiple reflections, and is especially efficient when $\tau$, the traveltime, is the same for all subdivisions. This type of model is a "Goupillaud medium", first discussed by Goupillaud (1961).

Klein-Gordon solutions $q$ for constant-dispersion density profiles may be computed through an extension of the Goupillaud algorithm. Klein-Gordon waves are propagated with the solution $e^{i \omega \tau(\omega)}$, where $\tau(\omega)$ is given by the thickness of the layer and

$$
v_{g}=c_{o} \sqrt{1-\frac{b_{o}^{2}}{\omega_{\mathrm{o}}^{2}}} .
$$

One obtains this expression for group (energy) velocity by substituting equation (2.14) into equation (2.16). Using this Klein-Gordon extension, a constant-dispersion layer 


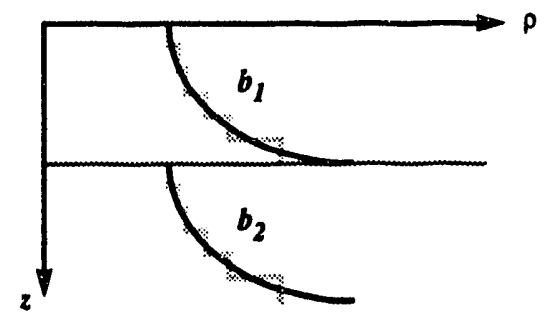

Fig. 3.1. Two different density parameterizations for variable-density media. Constantdispersion density profiles may be parameterized by their dispersion coefficient. This exponential example requires two dispersion values. For computational purposes one may also parameterize density functions as a sequence of constant-density subdivisions.

need not be subdivided further because this frequency-dependent solution imparts the desired waveform dispersion between interfaces.

Figure 3.2 shows the comparison between $p$ and $q$ solutions, computed with the Goupillaud algorithm, for the suite of density models in Figure 1.2. Again, speed was held constant at $3000 \mathrm{~m} / \mathrm{s}$ and the thickness over the halfspace is $18 \mathrm{un} \mathrm{m}$. Source and receiver are at the surface of the models. Part (c) establishes that the two solution types are numerically identical as long as the waveiength of density variation, $\Lambda_{\rho}$, is greater than the seismic wavelength. The density wavelength is measured from one density interface to the next in Figure 1.2.

I interpret the growing difference (up to $50 \%$ ) in amplitudes, for $\Lambda_{\rho} / \Lambda_{o}<1$, to be due to degradation of accuracy in the reflection coefficients used for the Klein-Gordon calculations (Figure 3.2b). Those coefficients were calcuiated with equation (7.33), though analytical work in that chapter associates this equation with small density gradients on either side of a discontinuity in density. The corresponding density profiles in Figure 1.2 obviously do not meet that criterion for accuracy when $\Lambda_{\rho} / \Lambda_{o}<1$. In deriving equation (7.33) I demonstrate that neither variable-density differential operator admits exact, Klein-Gordon scattering coefficients for this particular situation. I interpiet this limitation in the operators as the inevitable outcome of neglecting the gravitational force in the equations of motion. Because we neglect this force, we are saddled with the physics of a background medium in dynamic, rather than static, equilibrium.

Figure 3.3 helps establish the physical interpretation of the Klein-Gordon dispersion coefficient. These calculations are representative for models in Figure 1.2 having large ratios of $\Lambda_{\rho} / \Lambda_{o}$. These data suggest that we may interpret Klein-Gordon dispersion as that due to multiply-reflected energy generated by density variation in a medium. In this case, aùut ten constant-density subdivisions, for every constant-dispersion unit, produce enough multiple energy to accurately simulate the waveform dispersion through a single, continuous layer. A single $q$-type solution effectively imprints the same dispersion through the dispersion coefficient. 

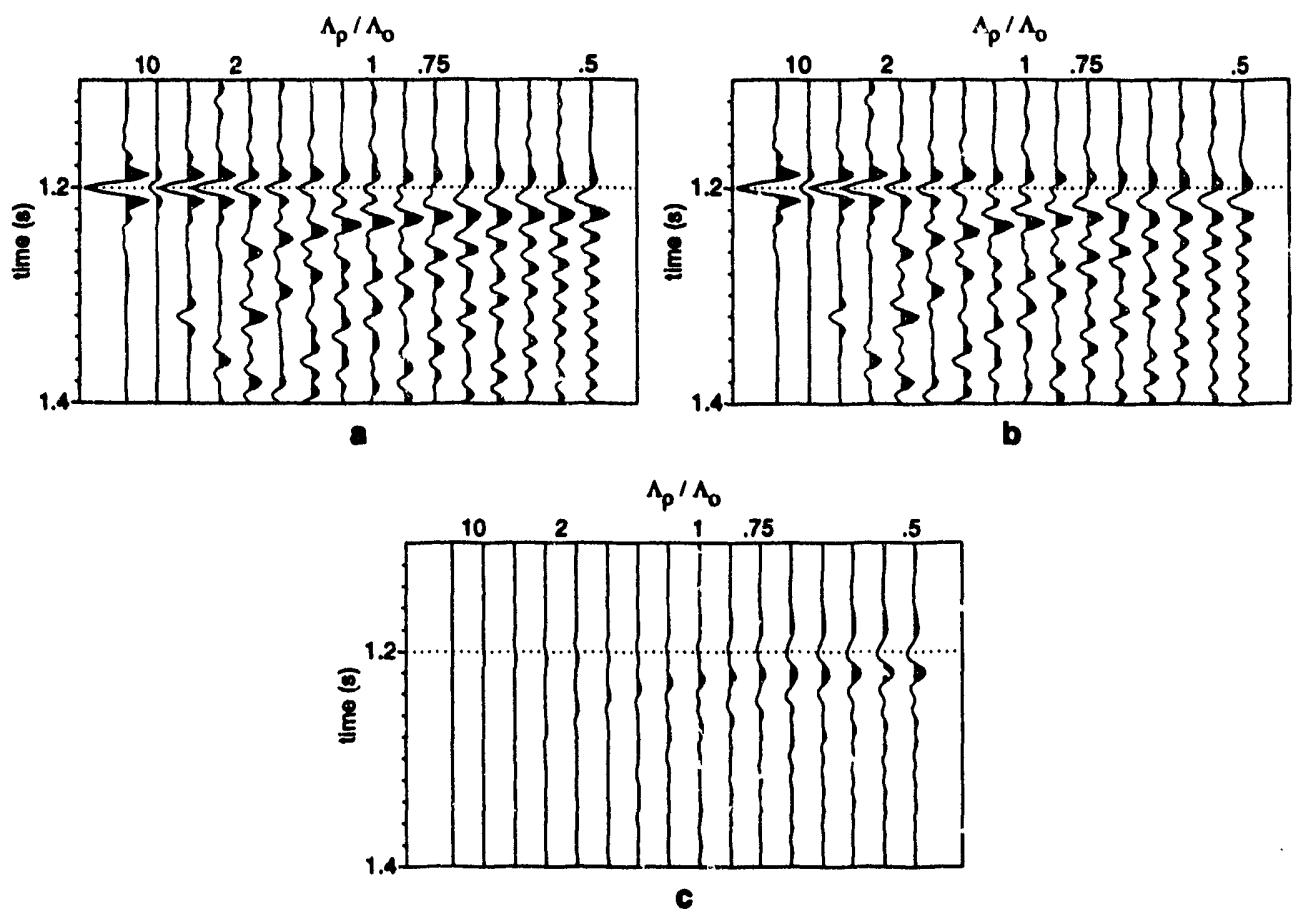

Fig. 3.2. Reflection data, including all multiples, computed to establish equivalency between variable-density and Klein-Gordon wave solutions. Each successive trace corresponds to a different one-dimensional model of density in Figure 1.2. The symbol $\Lambda_{\rho}$ denotes the wavelength of the density function, $\Lambda_{o}$ the seismic wavelength. These solutions were computed (a) as a composition of many elementary wave solutions, each computed for a constant-density subdivision of the density function and matched to the solution in the adjacent subdivision, and (b) as a composition of constant-dispersion Klein-Gordon solutions, with solution matching required only at points of discontinuous density in Figure 1.2. Part (c) displays the difference between the solutions in (a) and (b). 


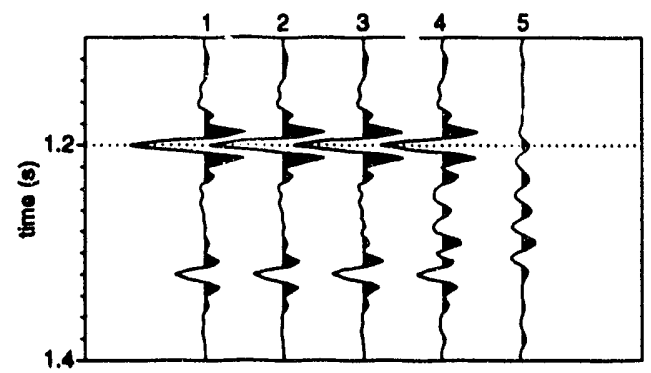

Fig. 3.3. A numerical experiment, computed to demonstrate the interpretation of KleinGordon dispersion as dispersion due to multiply-reflected energy. This test is for an exponential density function in Figure 1.2, with $\Lambda_{\rho} / \Lambda_{o}=3.75$. Trace (1) was computed using the Klein-Gordon description. Traces (2), (3), and (4) correspond, respectively, to 100,10 , and 5 constant-density subdivisions between discontinuities in the density function. Trace (5) is the difference between (3) and (4).

$\mathrm{O}$ Doherty and Anstey (1971) discuss this same dispersive phenomenon in terms of its effective time delay, though they do not formalize it in terms of a dispersion coeffcient. I will demonstrate, below, that the dispersion coefficient imparts dispersion due to continuous density variation, but does not account for the dispersion due to density discontinuities. It is the scattering coefficients that produce the latter type of dispersion. O'Doherty and Anstey do not distinguish between these dispersion types.

I pause to note the computational economy of the Klein-Gordon density parameterization. In this example, parameterization in terms of the dispersion coefficient requires an order of magnitude fewer values than model description in terms of density itself. We may postulate, therefore, that modeling computations with a Klein-Gordon formulation could be quite efficient for those limited geological settings where $c$ varies much more slowly than $\rho$. The dispersion coefficient may vary slowly over space (it is constant for our prototype density functions), though density varies rapidly. It is plausible, then, that Klein-Gordon model parameterization on a coarse grid would be efficient for those settings.

Figure 3.4 exemplifies the waveform response when $\Lambda_{\rho} / \Lambda_{o}$ is small. Here, five constant-density subdivisions of the continuous density units produce the same dispersion as ten, or even one hundred subdivisions. We may conclude, even in the face of inaccurate Klein-Gordon reflection coefficients, that dispersion due to continuous density variation is less important to the response. I attribute this outcome as the dominance of dispersion from density discontinuities-over that due to continuous density variation-when those discontinuities have small separation with respect to the seismic wavelength. The following experiments support this interpretation.

I have migrated, using the proper nominal values of dispersion for each density profile, the data in Figure 3.2 and plotted the images in Figure 3.5. Clearly, the halfspace 


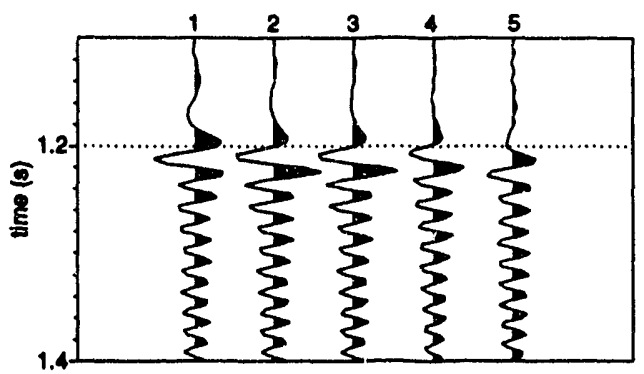

Fig. 3.4. A dispersion test with a rapidly-varying exponential density function, having $\Lambda_{\rho} / \Lambda_{o}=.5$. Trace (1) was computed using the Klein-Gordon description of propagation. Traces (2), (3), and (4) correspond, respectively, to 10, 5, and 2 constant-density subdivisions of a continuous segment of the density function. Trace (5) is the difference between (3) and (4).

has not been correctly imaged with thesc values, as it was in Figure 1.4. All traces should be identical to the trace at $b_{o}^{2} / \omega_{o}^{2}=0$. The input data for Figure 1.4 lack the dispersion due to multiple reflections between discontinuities. Comparing Figures 1.4 and 3.5 we see the importance of dispersion from discontinuities.

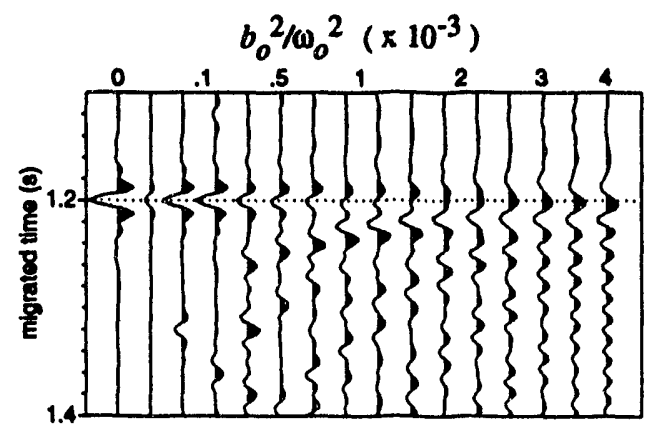

Fig. 3.5. Output data from Klein-Gordon phase-shift migration of the reflection data in Figure 3.2a. The values of $b_{o}^{2} / \omega_{o}^{2}$ indicate the (dimensionless) dispersion coefficient used for migration.

Let us consider another confirming experiment. We shall calculate data for models like those in Figure 1.2, but with quadratic, rather than exponential, density variation between interfaces. In particular, I choose a quadratic function,

$$
\rho(z)=a^{2} z^{2}+2 a \sqrt{\rho_{\min }} z+\rho_{\min },
$$

which is linear in $\sqrt{\rho} .\left(\sqrt{\rho}=a z+\sqrt{\rho_{\min }}\right)$ Dispersion $(\Delta \sqrt{\rho} / \sqrt{\rho})$ due to continuous 
density variation vanishes in this special case. Here,

$$
\begin{gathered}
a=\frac{\sqrt{\rho_{\min }}}{\delta z}(\sqrt{\alpha}-1), \\
\alpha=1+\frac{\delta \rho}{\rho_{\min }},
\end{gathered}
$$

and

$$
\delta \rho=\rho_{\max }-\rho_{\min }
$$

We will assign, as with the exponential functions, $\rho_{\min }=2 \mathrm{gm} / \mathrm{cm}^{3}$ at the top of each layer, $\rho_{\max }=3 \mathrm{gm} / \mathrm{cm}^{3}$ at the bottom, and require that $\delta z$ be the same layer thickness values as in Figure 1.2. Consider Figure 3.6 with this test in mind.

For small values of $\Lambda_{\rho} / \Lambda_{o}$-where the dispersion may be large enough to warrant Klein-Gordon migration-it essentially does not matter which density function is placed between discontinuities. Dispersive exponentials and nondispersive quadratics give the same response. The nominal value for dispersion between discontinuities therefore has nothing to do with the effective dispersion in these data. Migration with the nominal value will not be effective.

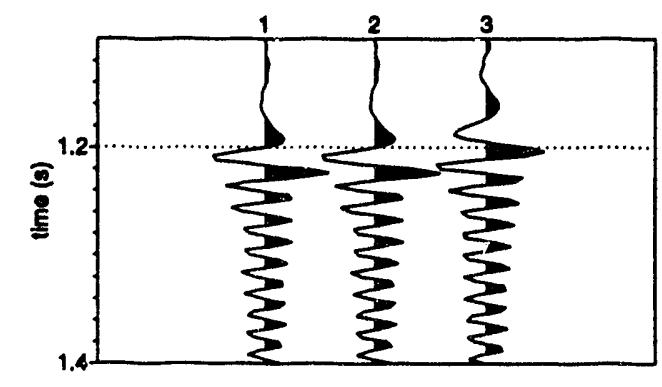

FIG. 3.6. A comparison to demonstrate the dominance of dispersion from density discontinuities over dispersion due to continuous density variation. Trace (1) is taken from the $\Lambda_{\rho} / \Lambda_{o}=.5$ position in Figure 3.2, for a highly-dispersive exponential density profile. Trace (2) comes from the same position, but for dispersionless quadratic profiles. They are essentially the same data. The migrated image (based on exponential-type dispersion) in trace (3) is not correct because the nominal dispersion between density discontinuities is overshadowed by the effective dispersion due to discontinuities.

All the data calculated for quadratic density profiles are plotted in Figure 3.7. Again, we note the inaccuracy of the Klein-Gordon solutions for $\Lambda_{\rho} / \Lambda_{o}<1$. 

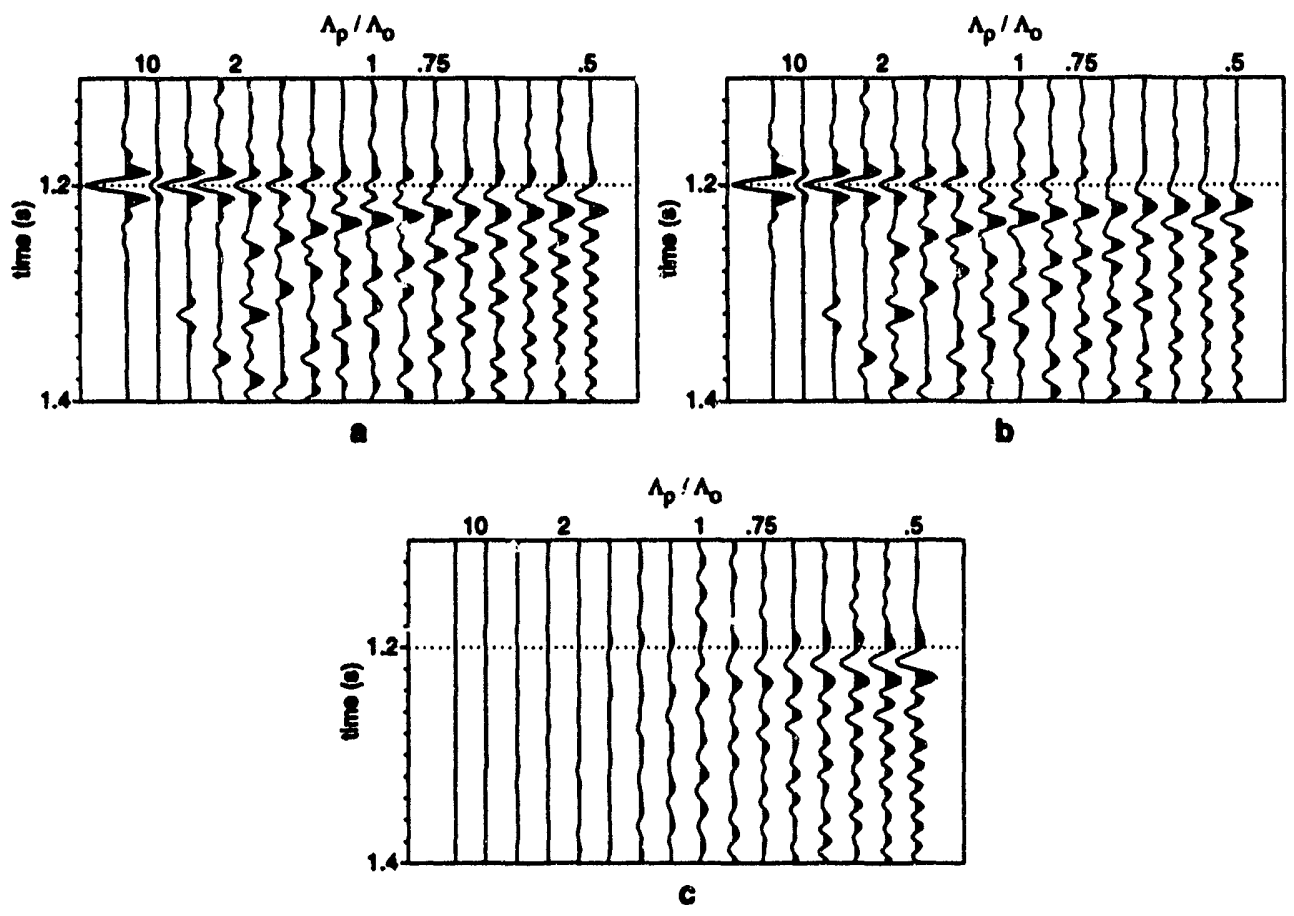

Fig. 3.7. Reflection data, including all multiple scattering, for a sequence of onedimensional models constructed from dispersionless quadratic density functions. The models are like those in Figure 1.2, except donsity varies quadratically, rather than exponentially, from 2 to $3 \mathrm{~g} / \mathrm{cm}^{3}$. The solutions were computed (a) in constant-density subdivisions of the density function and (b) in much larger constant-dispersion subdivisions. Part (c) displays the difference between the solutions in (a) and (b). 


\subsection{Profiles with discontinuous fractional density}

Let us try our luck with a ciass of constant-dispersion density profiles where density is continuous. This class is depicted in Figure 3.8. One might suppose in this case that dispersion from discontinuities will be less of a hindrance to imaging. We will not find this to be true. These models are discontinuous in the fractional density parameter, $\nabla \rho / \rho$.

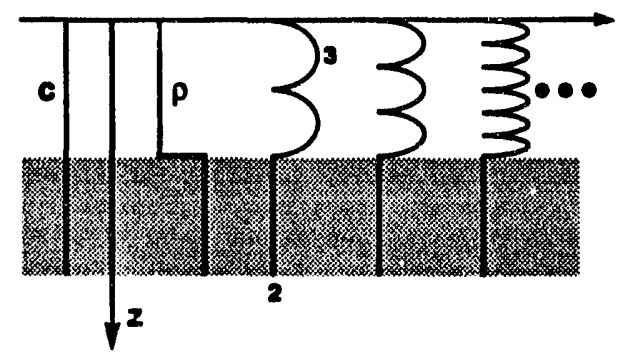

Fig. 3.8. A suite of density models, for analyzing how the rate of density variation affects wave propagation. The speed $c$ is held constant. With the exception of the first model, density $\rho$ varies sinusoidally between 2 and $3 \mathrm{~g} / \mathrm{cm}^{3}$ above a halfspace.

Figure 3.9 displays the data computed with the Goupillaud algorithm, using constantdensity subdivisions of the sinusoidal density profiles. (Equation (3.1) does not describe the energy velocity for these media, preventing the computation of a Klein-Gordon solution with Goupillaud's modeling algorithm.) I measure about a $.02 \mathrm{~s}$ time delay through

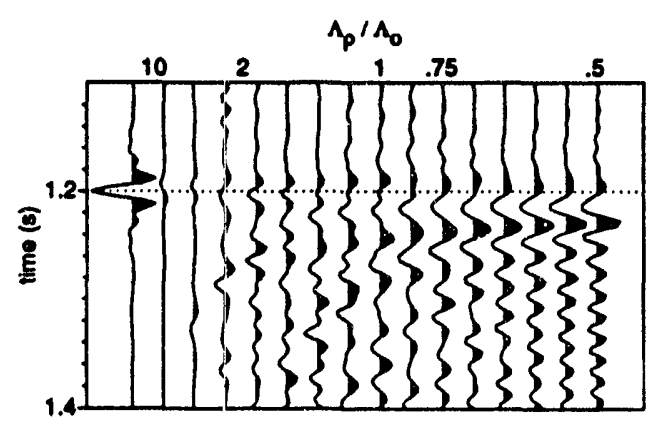

Fig. 3.9. Computed reflection data for the sequence of one-dimensional sinusoidal density models in Figure 3.8.

the most rapidly-varying density model. Knowing the nominal value for speed $(3000 \mathrm{~m} / \mathrm{s})$, this delay corresponds to a $30 \mathrm{~m}$ depth error - which we would like to migrate out. 
The images from these data are shown in Figure 3.10. Once again, Klein-Gordon mi-

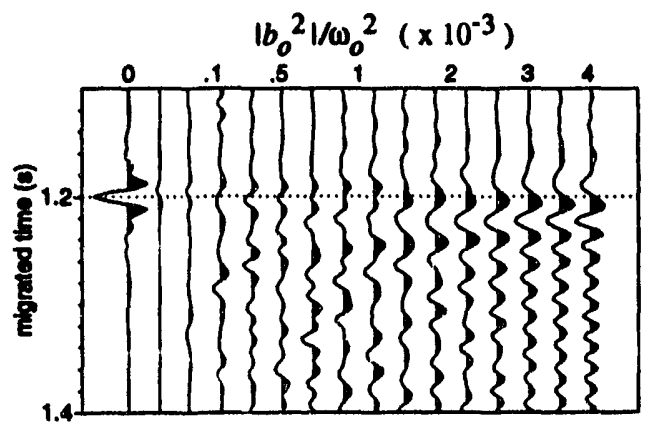

Fig. 3.10. Output data from Klein-Gordon phase-shift migration of the reflection data in Figure 3.9. The values of $\left|b_{o}^{2}\right| / \omega_{o}^{2}$ indicate the (dimensionless) dispersion coefficient used for migration.

gration did not image successfully the halfspace. Below, I derive an exact reflection coefficient (equation (7.28)) for these particular variable-density media. This new expression indicates that jumps in fractional density cause reflections (and thus dispersion)-though density itself is continuous.

I summarize the results of these experiments in terms of several practical conclusions. In the acoustic context, the Klein-Gordon dispersion coefficient may be interpreted as a vehicle that accounts for dispersion due to multiply-reflected energy-dispersion not accounted for with the constant-density wave operator. However, dispersion which originates from density discontinuities (of various types) is not described by this coefficient. Based on these prototype density models, it is, in all likelihood, the latter type of dispersion that plays the major role in seismic data. For this reason, simple extensions of our common imaging algorithms-from constant-density to Klein-Gordon-will not account properly for the time delays we know to expect. In order to account for these delays, an imaging algorithm must somehow incorporate a description of the scattering process, as well as the propagation process. 


\section{Chāpter 4}

\section{CAUSALITY AND ENERGY CONSERVATION}

Simply put, one computes causal solutions to equation (2.13) by mathematically constructing them to be so. For a real dispersion coefficient this construction falls within the normal bounds of experience but, for some, may seem out of bounds when that coefficient is imaginary. I will show that causality and energy conservation are equivalent principles, inextricably linked, justifying physically this construction. In particular, we will take causality as the first principle, and arrive at a statement of energy conservation.

Still, the analysis from which this statement of equivalency emerges has meaning only if the energy at some arbitrary time is guaranteed to be bounded. Hence, we shall also find conditions that preclude real, exponentially-growing solutions to general initialvalue problems based on equations (1.1) or (2.2).

\subsection{Causal solutions}

We may identify the construction required for causal solutions by first evaluating the integral over $\omega$ in equation (2.13). This integrand has zeros in its denominator at $\pm \omega(k)$, where

$$
\omega(k)=\sqrt{b_{o}^{2}+c_{o}^{2} k^{2}} .
$$

These zeros are plotted in Figures 4.1a, 4.1b, and 4.1c for real and imaginary dispersion coefficients and different ranges of wavenumber. For small wavenumbers and an imaginary dispersion coefficient these zeros migrate onto the imaginary $\omega$-axis, making insufficient the usual path of integration along the real $\omega$-axis. The integration path $C$ in Figure 4.1d produces a causal solution in all circumstances, as I now show.

We integrate along $C$ and close the contour in the upper half-plane of analyticity for $t<0$ and the lower half for $t>0$. A sum of residues produces

$$
\begin{aligned}
& \int_{C} d \omega {\left[\nu(k) * \frac{i \omega R(\boldsymbol{k})-S(\boldsymbol{k})-c_{o}^{2} F(\boldsymbol{k}, \omega)}{\omega^{2}-c_{o}^{2} k^{2}-b_{o}^{2}}\right] e^{-i \omega t}=} \\
& \pi H(t) \sum_{ \pm} \nu(\boldsymbol{k}) *\left[R(\boldsymbol{k}) \pm i \frac{S(\boldsymbol{k})+c_{o}^{2} F(\dot{\boldsymbol{k}}, \pm \omega(k))}{\sqrt{b_{o}^{2}+c_{o}^{2} k^{2}}}\right] e^{\mp i \sqrt{b_{o}^{2}+c_{o}^{2} k^{2} t}} .
\end{aligned}
$$

$H(t)$ is the Heaviside distribution

$$
H(t) \stackrel{\text { def }}{=} \begin{cases}0 & , t<0 \\ 1 & , t>0\end{cases}
$$



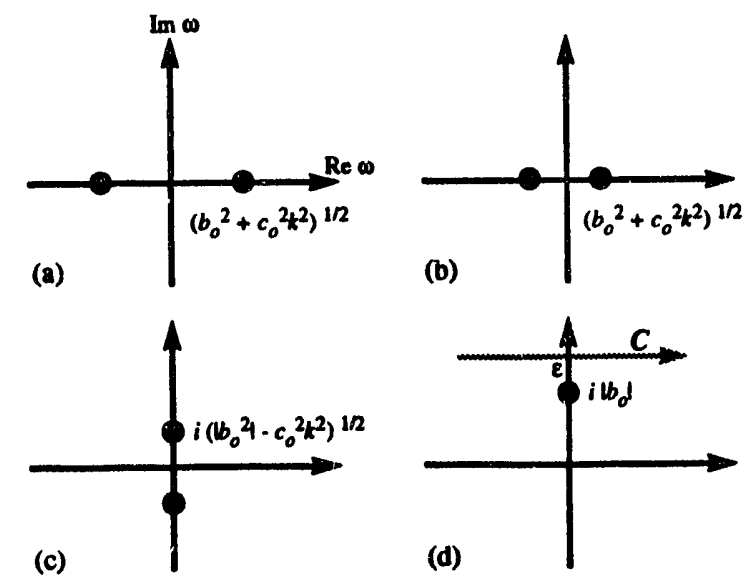

Fig. 4.1. Pole locations in the plane wave decomposition of a Klein-Gordon wave solution. Simple poles always lie on the real $\omega$-axis when (a) $b_{o}^{2}$ is positive or (b) when $b_{o}^{2}$ is negative but $k>\left|b_{o}\right| / c_{o}$. The poles reside on the imaginary axis when (c) $b_{o}^{2}$ is negative and $k<\left|b_{o}\right| / c_{o}$. The contour of integration $C$ in (d) produces the causal solution in all circumstances. This contour specifies an integration over all values of the real part of frequency, with the imaginary part of frequency being $\left|b_{o}\right|+\epsilon, \epsilon$ an arbitrarily small, real number.

Equation (4.2) gives the exact, causal solution for arbitrary initial data and source.

I emphasize that one must also incorporate an adequate imaginary part for frequency when using off-the-shelf Fourier transforms to solve, numerically, for the lefthand side of equation (4.2). This requirement becomes particularly obvious when $b_{o}^{2}<0$ and the initial data or the source function contain small wavenumbers $k<\left|b_{o}\right| / c_{o}$. The numerical solution is noncausal at these wavenumbers unless $\Im(\omega)>\sqrt{\left|b_{o}\right|^{2}-c_{o}^{2} k^{2}}$. Effectively, the Fourier transform sees a pole for $t<0$, and the associated residue is not zero. $\Im(\omega)>\left|b_{o}\right|$ suffices to enforce causality for all wavenumbers.

By insertion of equation (4.2) into equation (2.13),

$$
\begin{aligned}
q(x, t)= & \frac{1}{(2 \pi)^{3}} H(t) \sum_{ \pm} \int_{-\infty}^{\infty} d k \nu(k) \\
& *\left[R(k) \pm i \frac{S(k)+c_{o}^{2} F(k, \pm \omega(k))}{\sqrt{b_{o}^{2}+c_{o}^{2} k^{2}}}\right] e^{i\left(k \cdot x \mp \sqrt{b_{o}^{2}+c_{o}^{2} k^{2}} t\right)} .
\end{aligned}
$$

From our analysis of poles we may also refine equations $(2.10)$ as

$$
\lim _{t \rightarrow \infty} q=O\left(e^{\left(\left|b_{b}\right|+\epsilon\right) t}\right), \quad \lim _{|x| \rightarrow \infty}(q,|\nabla q|)=0 .
$$

Let us now specify an example where equation (4.4) can be evaluated analytically. 
As an example we shall find an analytic representation of the data in Figure 1.1. We first assume that

$$
r(x)=\delta\left(x-x^{\prime}\right), \quad s(x)=0, \quad f(x, t)=0 .
$$

From equation (2.8)

$$
R(\boldsymbol{k})=e^{-i \boldsymbol{k} \cdot \boldsymbol{x}^{\prime}} .
$$

We see immediately that this choice of $r(x)$ permits solution for an arbitrary density profile. Equation (4.4) simplifies to

$$
\begin{aligned}
q(x, t) & =\frac{1}{(2 \pi)^{3}} H(t) \sum_{ \pm} \int_{-\infty}^{\infty} d k\left[\nu(k) * e^{-i k \cdot x^{\prime}}\right] e^{i\left[k \cdot x \mp \sqrt{b_{o}^{2}+c_{o}^{2} k^{2}} t\right]} \\
& =\frac{1}{(2 \pi)^{3}} \frac{1}{\sqrt{\rho\left(x^{\prime}\right)}} H(t) \sum_{ \pm} \int_{-\infty}^{\infty} d k e^{i\left[k \cdot\left(x-x^{\prime}\right) \mp \sqrt{\left.b_{o}^{2}+c_{o}^{2} k^{2} t\right]}\right.}
\end{aligned}
$$

by employing our definition of convolution (equation (2.12)) and equation (2.11). Now, I specialize equation (4.7) to a one-dimensional problem in the coordinate $z$, to match the suite of experiments represented in Figure 1.2. That is,

$$
q(z, t)=\frac{1}{2 \pi} \frac{1}{\sqrt{\rho\left(z^{\prime}\right)}} H(t) \sum_{ \pm} \int_{-\infty}^{\infty} d k_{z} e^{i\left[k_{z}\left(z-z^{\prime}\right) \mp \sqrt{\left.b_{0}^{2}+c_{o}^{2} k_{z}^{2} t\right]}\right.}
$$

Derived in Appendix B,

$$
\begin{aligned}
& q(z, t)=\frac{1}{\sqrt{\rho\left(z^{\prime}\right)}} H(t) \frac{c_{o} t}{\zeta}\left[-2 \delta\left(\zeta^{2}\right)+\frac{1}{2} \frac{b_{o}}{c_{o}} H\left(\zeta^{2}\right) J_{1}\left(\frac{b_{o}}{c_{o}} \zeta\right)\right] \\
& \zeta \stackrel{\text { def }}{=} \sqrt{c_{o}^{2} t^{2}-\left(z-z^{\prime}\right)^{2}}, \quad \zeta>0, \quad b_{o}^{2}>0 .
\end{aligned}
$$

$J_{1}$ is the Bessel function of the first kind and order one. This Bessel function may be replaced by a cosine when its argument is large (Abramowitz and Stegun, 1965), and we may also write

$$
q(z, t) \sim \frac{1}{\sqrt{\rho\left(z^{\prime}\right)}} H(t) \frac{c_{o} t}{\zeta}\left[-2 \delta\left(\zeta^{2}\right)+\sqrt{\frac{1}{2 \pi} \frac{b_{o}}{c_{o}}} H\left(\zeta^{2}\right) \frac{1}{\sqrt{\zeta}} \cos \left(\frac{b_{o}}{c_{o}} \zeta-\frac{3 \pi}{4}\right)\right] .
$$

The Klein-Gordon wavefields of equations (4.9) and (4.10) describe real, outgoing waveforms, as one would expect from equation (4.6). These waveforms are composed of two components, one non-dispersive and the other dispersive. The non-dispersive component is a propagating singularity, $\delta$, present whether or not the medium is dispersive. The second, oscillating component is responsible for the dispersion in Figure 1.1. This dispersive component vanishes when $b_{o}=0$. 
Importantly, the propagating singularity has its support at $\zeta=0$. In this respect, the data of Figure 1.1 differ from equations (4.9) and (4.10). This difference occurs because our analytic solution has infinite bandwidth (equation (4.6)), and those data do not. From equation (2.16), only large wavenumbers propagate at speed $c_{o}$. Hence, bandlimited data are not supported at $\zeta=0$ but, instead, exhibit a time delay. This delay is not clearly evident in Figure 1.1 due to the (non-causal) zero-phase wavelet.

Let us consider equation $(4.8)$ for $b_{o}^{2}<0$. In this case we write that

$$
\begin{aligned}
& q(z, t)=\frac{1}{2 \pi} \frac{1}{\sqrt{\rho\left(z^{\prime}\right)}} H(t) \sum_{ \pm} \\
& {\left[\int_{-\infty}^{-\left|b_{0}\right| / c_{0}} d k_{z} e^{i\left[k_{z}\left(z-z^{\prime}\right) \mp \sqrt{b_{0}^{2}+c_{0}^{2} k_{z}^{2}} t\right]}\right.} \\
& +\int_{-\left|b_{0}\right| / c_{o}}^{\left|b_{0}\right| / c_{0}} d k_{z} e^{i k_{z}\left(z-z^{\prime}\right)} e^{\mp \sqrt{\left|b_{o}^{2}\right|-c_{o}^{2} k_{z}^{2} t}} \\
& \left.+\int_{\left|b_{0}\right| / c_{0}}^{\infty} d k_{z} e^{i\left[k_{z}\left(z-z^{\prime}\right) \mp \sqrt{b_{o}^{2}+c_{o}^{2} k_{z}^{2} t}\right]}\right] \text {. }
\end{aligned}
$$

The solution is not wavelike over the entire $k_{z}$-band but, instead, contains real exponentials (growing and decaying) over time for $-\left|b_{o}\right| / c_{o}<k_{z}<\left|b_{o}\right| / c_{o}$. Moreover, since density $\rho$ is sinusoidal in this case, $p$-not just $q$-becomes unbounded over time for this band of wavenumbers. (Recall that $p=\sqrt{\rho} q$.) Of course, these troublesome, small wavenumbers may well be absent in most real data.

I underscore, for the sake of the discussion to come, that equation (4.11) is a valid (though unstable) solution of the original initial value problem defined by equations (1.1) and (1.2), or equations (2.6). I use the word valid in the sense that equation (4.11) satisfies the required limits on a solution, equations (4.5). The fastest exponential growth in equation (4.11) occurs for $k_{z}=0$, giving growth like $O\left(e^{\left|b_{o}\right| t}\right)$. Equations (4.5) permit, albeit with no room to spare, this order of growth. Our beginning requirements on solutions are thus met by equation (4.11).

\subsection{Well-posed problems}

Motivated by equation (4.11) I examine how to pose Klein-Gordon (equivalently, variable-density) initial-value problems in a way that excludes real, exponential solutions. I will show that conditions for well-posedness differ, depending on the sign of $b_{o}^{2}$. In particular, when $b_{o}$ is imaginary, real exponentials will always arise-for that class of problems that specify initial data on the surface $t=0$. This irritation can be avoided for more general classes of problems, like those involving moving sources. Hence, I judge that exponentially-growing solutions are not always a reflection of a non-physical process. In this instance they are related to how initial data were defined.

To develop conditions for well-posedness one may assume the opposite. I postulate 
a Klein-Gordon solution

$$
q=e^{ \pm \Sigma+i K}
$$

that involves both oscillatory and real exponentials. Four-vectors simplify notation. The hypersurfaces on which we shall prescribe initial data are given by $\Sigma=C_{1}, K=C_{2},\left(C_{1}\right.$ and $C_{2}$ constants) where

$$
\begin{aligned}
\Sigma & \stackrel{\text { def }}{=} \Sigma_{4} \cdot x_{4} \\
& =\Sigma \cdot x-\Sigma_{0} t
\end{aligned}
$$

and

$$
\begin{aligned}
K & \stackrel{\text { def }}{=} k_{4} \cdot x_{4} \\
& =k \cdot x-\omega t .
\end{aligned}
$$

Here,

$$
x_{4} \cdot x_{4} \stackrel{\text { def }}{=} x \cdot x-c_{o}^{2} t^{2} .
$$

One may interpret $\left(\Sigma, \Sigma_{o} / c_{o}\right)$ and $\left(\boldsymbol{k}, \omega / c_{o}\right)$ as components of four-vectors normal to surfaces $\Sigma$ and $K$. In what follows I will take the components of $\Sigma$ and $K$ as constants, thus defining planar surfaces. In general, however, $\Sigma$ and $K$ represent arbitrary spacetime surfaces, when set equal to some constant.

Let us give an example before proceeding further. Consider a problem where $q$ satisfies

$$
\Delta q-\frac{b^{2}}{c^{2}} q-\frac{1}{c^{2}} \frac{\partial^{2} q}{\partial t^{2}}=0
$$

with

$$
q(x, t)=C
$$

on the surface $\Sigma=0, C$ a constant. The general solution is

$$
q=A e^{\Sigma} \cos K+B e^{\Sigma} \sin K
$$

under conditions stated below. The initial data require that $A=C$ and $K=\Sigma$. The constant $B$ can be determined by specifying the derivative of $q$ in the direction normal to the hypersurface $\Sigma=0$.

I emphasize two things about this example. Apparently, it is not difficult to create an initial value problem with an exponentially growing solution. Secondly, we will think of $\Sigma$ as a surface upon which one specifies initial data. To specify data on the surface $t=0$ one takes $\boldsymbol{\Sigma}=\mathbf{0}$ and $C_{1}=0$.

Dual requirements come forth upon substitution of equation (4.12) into equation (4.15). 
From real and imaginary parts,

$$
\begin{aligned}
& \boldsymbol{\Sigma}_{4} \cdot \boldsymbol{\Sigma}_{4}=\boldsymbol{k}_{4} \cdot \boldsymbol{k}_{4}+\frac{b_{o}^{2}}{c_{o}^{2}} \\
& \boldsymbol{\Sigma}_{4} \cdot \boldsymbol{k}_{4}=0
\end{aligned}
$$

It is important to recognize that the " \pm " symbol we began with in equation (4.12) has dropped out of the analysis. That is to say, conditions are identical for growing or declining exponential solutions.

We may unify the requirements of equations (4.16) by eliminating, through the bottom equation of the pair, $\omega$ in favor of

$$
\begin{gathered}
\omega=\frac{c_{o}^{2}}{\Sigma_{o}} k \cdot \Sigma \\
\Sigma_{4} \cdot \Sigma_{4}-k \cdot k+\frac{c_{o}^{2}}{\Sigma_{o}^{2}}(k \cdot \Sigma)^{2}=\frac{b_{o}^{2}}{c_{o}^{2}}
\end{gathered}
$$

But

$$
(\boldsymbol{k} \cdot \boldsymbol{\Sigma})^{2} \leq(\boldsymbol{k} \cdot \boldsymbol{k})(\boldsymbol{\Sigma} \cdot \boldsymbol{\Sigma})
$$

by the Cauchy-Schwartz inequality.

We can now say that the inequality

$$
\Sigma_{4} \cdot \Sigma_{4}\left[1+\frac{c_{o}^{2}}{\Sigma_{o}^{2}} k \cdot k\right] \geq \frac{b_{o}^{2}}{c_{o}^{2}}
$$

ensures a real, exponential factor in $q$, so

$$
\boldsymbol{\Sigma}_{4} \cdot \boldsymbol{\Sigma}_{4}<\frac{b_{o}^{2}}{c_{o}^{2}}\left[\frac{1}{1+\left(c_{o}^{2} / \Sigma_{o}^{2}\right) k \cdot k}\right]
$$

therefore precludes that possibility. The bounds

$$
0 \leq\left[\frac{1}{1+\left(c_{o}^{2} / \Sigma_{o}^{2}\right) k \cdot k}\right] \leq 1
$$

on the bracketed term of equation (4.17) further simplify the condition for well-posedness. From these bounds and inequality (4.17),

$$
\boldsymbol{\Sigma}_{4} \cdot \boldsymbol{\Sigma}_{4}< \begin{cases}0 & , b_{o}^{2}>0 \\ b_{o}^{2} / c_{o}^{2} & , b_{o}^{2}<0 \\ 0 & , \forall b_{o}^{2}, \boldsymbol{k} \cdot \boldsymbol{k} \rightarrow \infty\end{cases}
$$


Inequalities (4.18) itemize how Klein-Gordon initial-value problems must be posed for oscillatory solutions. If $b_{o}^{2}>0$,

$$
\left(|\Sigma|+\frac{\left|\Sigma_{o}\right|}{c_{o}}\right)\left(|\Sigma|-\frac{|\Sigma o|}{c_{o}}\right)<0 \quad \Longrightarrow|\Sigma|<\frac{|\Sigma o|}{c_{o}} .
$$

This inequality is satisfied trivially on a surface of constant time, but never on a surface of constant depth. Hence, wave operators that, say, downward continue data (Claerbout, 1985) from one depth to another will always admit real, exponentially-growing solutions for some initial data.

Furthermore, initial data can always be set at $t=0$ such that, if $b_{o}^{2}<0$, the solution $q$ will grow exponentially. For this case, inequalities (4.18) specify that $\Sigma_{o}>\left|b_{o}\right|$. We must then require that a space-time surface $-\Sigma_{o} t=C_{1}$ satisfies

$$
t<\frac{C_{1}}{\left|b_{o}\right|}
$$

to avoid real exponentials. This inequality cannot be met for the surface $t=0$ (take $C_{1}=0$ ) if the field is required to be zero for $t<0$. Moreover, the problems defined in equations (1.1) and (1.2), or equations (2.6), reside in this category. The real exponentials of equation (4.11) are the necessary outcome.

I also record as the last case of inequalities (4.18) the condition for well-posedness at large wavenumbers. From equation (4.17), the sign of $b_{o}^{2}$ does not matter. Klein-Gordon, or valiable-density, solutions obtained from data posed at $t=0$ are always oscillatory at large wavenumbers, as in equation (4.11). For the example in equation (4.11) we see that "large" means $\left|k_{z}\right|>\left|b_{o}\right| / c_{o}$.

\subsection{Energy conservation}

The aim here is threefold. All the while assuming a well-posed initial-value problem, I will connect causality with energy conservation as mutually inclusive principles. This means that any mathematical construction employed to enforce causality has a sound physical underpinning in terms of energy conservation. A second, intertwined issue in that connection is the choice of a variable-density operator, with or without the term involving the divergence of fractional density. An operator that includes that term conserves energy without fail. I will also demonstrate that Klein-Gordon operations conserve the acoustic energy contained in the physical field, $p$.

We begin by formulating, from the differential operators, differential forms of Poynting's theorem (Auld, 1990) for acoustic fields. We will integrate these exact differentials over a particular space-time domain to calculate the acoustic energy in a field.

The operator on velocity potential $\psi$ provides the most convenient starting point. 
The fields $p, q$, and $\psi$ are related as

$$
p=\sqrt{\rho} q=\rho \frac{\partial \psi}{\partial t} .
$$

Derived in equation (A-25) of Appendix A,

$$
L^{(\psi)} \stackrel{\text { def }}{=} \frac{1}{\rho} \nabla \cdot(\rho \nabla \psi)-\frac{1}{c^{2}} \frac{\partial^{2} \psi}{\partial t^{2}} .
$$

One develops Poynting's theorem from $L^{(\psi)}$ by multiplying by $\rho(\partial \psi / \partial t)$. That is,

$$
\rho \frac{\partial \psi}{\partial t} L^{(\psi)} \psi=\nabla \cdot\left[\rho \frac{\partial \psi}{\partial t} \nabla \psi\right]-\frac{\partial}{\partial t}\left[\frac{1}{2} \frac{\rho}{c^{2}}\left(\frac{\partial \psi}{\partial t}\right)^{2}+\frac{1}{2} \rho(\nabla \psi)^{2}\right] .
$$

To get this result it helps to recognize that

$$
\frac{\partial}{\partial t}(\nabla \psi)^{2}=2 \nabla \psi \cdot \frac{\partial}{\partial t}(\nabla \psi)
$$

and

$$
\frac{\partial}{\partial t}\left(\frac{\partial \psi}{\partial t}\right)^{2}=2 \frac{\partial \psi}{\partial t} \frac{\partial^{2} \psi}{\partial t^{2}} .
$$

We may easily interpret equation (4.21) upon converting it to the physical variables $\boldsymbol{v}$ and $p$, where $v$ denotes a perturbation in particle velocity from a background field. From equations (4.19) and

$$
\begin{gathered}
v=-\nabla \psi \\
\rho \frac{\partial \psi}{\partial t} L^{(\psi)} \psi=-\nabla \cdot(p v)-\frac{\partial}{\partial t}\left(\frac{1}{2} \frac{1}{\rho c^{2}} p^{2}+\frac{1}{2} \rho v \cdot v\right) \\
\stackrel{\text { def }}{=} \nabla \cdot P-\frac{\partial E_{v}}{\partial t} .
\end{gathered}
$$

The divergence operator is on the Poynting vector $\boldsymbol{P}$, an energy flux vector. The time derivative operates on the total energy density $\left(E_{v}\right)$ in the field, given by the sum of potential and kinetic energy densities. The lefthand side of equations (4.23) is zero if there is no source term in the balance of forces, and we obtain a statement of energy conservation; the energy flux (out of a volume) equals the time rate of change of energy (in that volume).

Now we form the same energy expression with operators for $p$ and $q$. Defining $L^{(p)}$ from equation (1.1),

$$
L^{(p)} \stackrel{\text { def }}{=} \rho \nabla \cdot\left[\frac{\nabla p}{\rho}\right]-\nabla \cdot\left[\frac{\nabla \rho}{\rho}\right] p-\frac{1}{c^{2}} \frac{\partial^{2} p}{\partial t^{2}},
$$


or

$$
L^{(p)}=\rho L^{(\psi)} \frac{\partial \psi}{\partial t}
$$

from the relation between $p$ and $\psi$ (equations (4.19)) and the definition of $L^{(\psi)}$ (equation (4.20)). We can manipulate this new expression for $L^{(p)}$, written in terms of $L^{(\psi)}$, into the lefthand-side of equation (4.23). By doing so we will gain Poynting's theorem again, but in terms of $p$ rather than $\psi$.

Commuting the time and space operators in equation (4.25), integrating over time, then multiplying by $\partial \psi / \partial t$,

$$
\frac{p}{\rho} L^{(r)} p^{(t)}=\rho \frac{\partial \psi}{\partial t} L^{(\psi)} \psi
$$

The symbol $p^{(t)}$ denotes the time integration,

$$
p^{(t)} \stackrel{\text { def }}{=} \int^{t} d t^{\prime} p
$$

These operations guarantee that the lefthand side of equation (4.26) equals the desired energy expression, the righthand side of equation (4.23).

We follow the same pattern for energy in terms of $q$. Taking

$$
L^{(q)} \stackrel{\text { def }}{=} \Delta q-\frac{b^{2}}{c^{2}} q-\frac{1}{c^{2}} \frac{\partial^{2} q}{\partial t^{2}}
$$

one derives

$$
q L^{(q)} q^{(t)}=\rho \frac{\partial \psi}{\partial t} L^{(\psi)} \psi
$$

with

$$
q^{(t)} \stackrel{\text { def }}{=} \int^{t} d t^{\prime} q
$$

Collecting results,

$$
\begin{aligned}
\rho \frac{\partial \psi}{\partial t} L^{(\psi)} \psi & =\frac{p}{\rho} L^{(p)} p^{(t)} \\
& =q L^{(q)} q^{(t)} \\
& =\nabla \cdot P-\frac{\partial E_{v}}{\partial t}
\end{aligned}
$$

Equations (4.29) provide a convenient apparatus to establish a link between causality and energy conservation. With these equations we may consider simultaneously the acoustic energy density $E_{v}$ that resides in any of three fields $p, q$, or $\psi$.

Let us integrate equations (4.29) over a space-time domain $D$, depicted in.Figure 4.2 for two spatial dimensions. This figure embodies the following experiment. We shall pose initial data on the general space-time surface $B_{1}$, as per the discussion of well-posed initial value problems. These data propagate out into $D$ via any of the three operators $L^{(q)}$, $L^{(p)}$, or $L^{(\psi)}$. We will then observe the energy in those data on the surface $B_{2}$, at some 


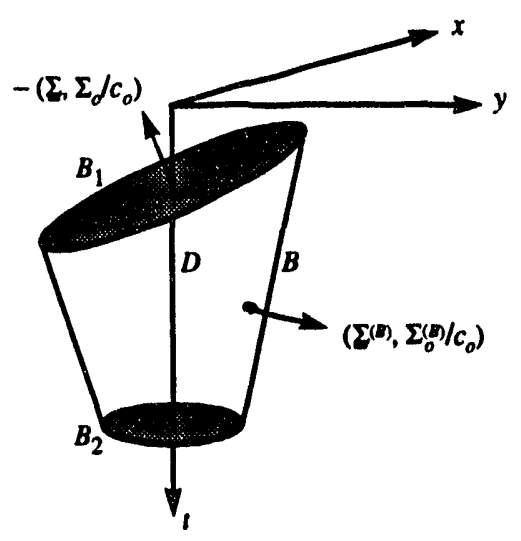

Fig. 4.2. A space-time domain $D$ of integration, for tracking propagating energy over time. Symbols $B_{1}, B$, and $B_{2}$ denote the boundaries of $D$. Vector normals to $B_{1}$ and $B$ are shown. $B_{2}$ is a region of space at $t=t_{2}$.

later time $t_{2}$.

The area (volume in three dimensions) of $B_{2}$ and, hence, the sides $B$ of this domain will be limited in a way that enforces causality. Characteristic speed $c$ sets the maximum speed for the transfer of energy in the data on $B_{1}$. Hence, the choice of $t_{2}$ dictates the maximum area $B_{2}$ of space that may contain causal energy.

Let us name the integral,

$$
E \stackrel{\text { deff }}{=} \int_{D} d V d t\left(\nabla \cdot P-\frac{\partial E_{v}}{\partial t}\right),
$$

where $d V$ denotes an area (volume) element of $D$. Since $V$ depends on time I rewrite equation (4.30) in four-vector notation to simplify the calculation. This rewritten form,

$$
c_{o} E=\int_{D} d V d\left(c_{o} t\right) \nabla_{4} \cdot\left(\boldsymbol{P}, c_{o} E_{v}\right),
$$

permits a simple application of the divergence theorem. The operator $\nabla_{4}$ has the definition

$$
\nabla_{4} \stackrel{\text { def }}{=}\left(\nabla, \frac{1}{c_{o}} \frac{\partial}{\partial t}\right)
$$

By the divergence theorem,

$$
\begin{aligned}
c_{o} E= & \int_{B_{1}} d S\left(\boldsymbol{P}, c_{o} E_{v}\right) \cdot \hat{\boldsymbol{n}}_{D}+\int_{B_{2}} d S\left(\boldsymbol{P}, c_{o} E_{v}\right) \cdot \hat{\boldsymbol{n}}_{D} \\
& +\int_{B} d S\left(\boldsymbol{P}, c_{o} E_{v}\right) \cdot \hat{\boldsymbol{n}}_{D} .
\end{aligned}
$$


Assisted by Figure 4.2 I find that

$$
\hat{n}_{D}= \begin{cases}-\Sigma_{4} /\left|\Sigma_{4}\right| & , \text { on } B_{1} \\ \Sigma_{4}^{(B)} /\left|\Sigma_{4}^{(B)}\right| & , \text { on } B \\ (0,1) & , \text { on } B_{2}\end{cases}
$$

and

$$
d S= \begin{cases}\left(c_{o} / \Sigma_{o}\right)\left|\Sigma_{4}\right| d V & , \text { on } B_{1} \\ \left(c_{o} / \Sigma_{o}^{(B)}\right)\left|\Sigma_{4}^{(B)}\right| d V & , \text { on } B \\ d V & , \text { on } B_{2}\end{cases}
$$

Placing the substitutions for $\hat{\boldsymbol{n}}_{D}$ into equation (4.32) and dividing through by $c_{o}$,

$$
\begin{aligned}
E= & \frac{1}{c_{o}} \int_{B_{1}} d S \frac{1}{\left|\Sigma_{4}\right|}\left(-\boldsymbol{P} \cdot \boldsymbol{\Sigma}+\Sigma_{o} E_{v}\right)-\int_{B_{2}} d V E_{v} \\
& +\frac{1}{c_{o}} \int_{B} d S \frac{1}{\left|\Sigma_{4}^{(B)}\right|}\left(\boldsymbol{P} \cdot \Sigma^{(B)}-\Sigma_{o}^{(B)} E_{v}\right) .
\end{aligned}
$$

We have reduced the expression for $E$ down to two integrals over space-time and the energy in the acoustic field at time $t_{2}$. We can make further reductions to equation (4.35) by estimating those two integrals such that an upper bound on $E$ is obtained. In particular, I want an upper bound that is related to $E_{v}$, the energy density.

I will first estimate $\left|\boldsymbol{P} \cdot \boldsymbol{\Sigma}^{(B)}\right|$. Let us express this magnitude in, say, terms of $\boldsymbol{q}$. From equations (4.19), (4.22), and (4.23),

$$
\begin{aligned}
\boldsymbol{P} & =-p v \\
& =\sqrt{\rho} q \nabla\left(\frac{1}{\sqrt{\rho}} q^{(t)}\right) .
\end{aligned}
$$

We may now estimate

$$
\begin{aligned}
\left|\sqrt{\rho} q \Sigma^{(B)} \cdot \nabla\left(\frac{1}{\sqrt{\rho}} q^{(t)}\right)\right| & \leq\left|\Sigma^{(B)}\right||q|\left|\sqrt{\rho} \nabla\left(\frac{1}{\sqrt{\rho}} q^{(t)}\right)\right| \\
& =c\left|\Sigma^{(B)}\right|\left|\frac{q}{c}\right|\left|\sqrt{\rho} \nabla\left(\frac{1}{\sqrt{\rho}} q^{(t)}\right)\right| \\
& \leq \frac{c}{2}\left|\Sigma^{(B)}\right|\left\{\frac{q^{2}}{c^{2}}+\rho\left[\nabla\left(\frac{1}{\sqrt{\rho}} q^{(t)}\right)\right]^{2}\right\} \\
& =c\left|\Sigma^{(B)}\right| E_{v} .
\end{aligned}
$$

The first bound follows from the Cauchy-Schwartz inequality, the second bound comes 
from the expansion of

$$
\left[\frac{q}{c} \pm\left|\sqrt{\rho} \nabla\left(\frac{1}{\sqrt{\rho}} q^{(t)}\right)\right|\right]^{2}
$$

and the last line from equations (4.19), (4.22), and (4.23). Equations (4.36) establish simply that

$$
\boldsymbol{P} \cdot \boldsymbol{\Sigma}^{(B)} \leq c\left|\boldsymbol{\Sigma}^{(B)}\right| E_{v}
$$

and we may again dispense with the need to spuak explicitly in terms of $p, q$, or $\psi$.

Using the estinate for $\left|\boldsymbol{P} \cdot \boldsymbol{\Sigma}^{(B)}\right|$ and, similarly, another for $|\boldsymbol{P} \cdot \boldsymbol{\Sigma}|$,

$$
\begin{aligned}
E \leq & -\frac{1}{c_{o}} \int_{B_{1}} d S \frac{c}{\left|\Sigma_{4}\right|}\left[|\Sigma|-\frac{\Sigma_{o}}{c}\right] E_{v}-\int_{B_{2}} d V E_{v} \\
& +\frac{1}{c_{o}} \int_{B} d S \frac{c}{\left|\Sigma_{4}^{(B)}\right|}\left[\left|\Sigma^{(B)}\right|-\frac{\Sigma_{o}^{(B)}}{c}\right] E_{v}
\end{aligned}
$$

from equation (4.35). To enforce causality I follow Bleistein (1984) in requiring that

$$
\left|\Sigma^{(B)}\right|-\frac{\Sigma_{0}^{(B)}}{c} \leq 0
$$

Before I explain this requirement, observe its consequence. Now $E$ in inequality (4.38) is bounded by the integral over $B_{1}$ and the energy at time $t_{2}$. (Inequality (4.39) makes the integral over $B$ a nonpositive number.)

Bleistein derives that the characteristic (light) cone for the constant-density wave equation is tangent at every point to

$$
\frac{1}{c^{2}}\left[\frac{\partial \Sigma^{(B)}}{\partial t}\right]^{2}=\left[\nabla \Sigma^{(B)}\right]^{2}
$$

This single cone defines the boundary of the domain of dependence of wave solutions $p, q$, and $\psi$. That is to say, by restricting $D$ to the interior of this cone we limit our integration to a dumain that contains only causal energy. $\Sigma^{(B)}=$ constant represents the hypersurface $B$ of Figure 4.2 , where the components $\left(\Sigma^{(B)}, \Sigma_{o}^{(B)}\right)$ give the normal to $B$ at any point.

We ma- write an expression for a local tangent plane, tangent to the characteristic cone at an arbitrary point, by doing the derivatives of equation (4.40). These derivatives give

$$
\left|\Sigma^{(B)}\right|^{2}-\frac{\left(\Sigma_{o}^{(B)}\right)^{2}}{c^{2}}=0
$$

One sees, by factoring equation (4.41), that inequality (4.39) defines the interior of the light cone.

Alluded to above, the same cone applies to each of the wave solutions $p, q$, and $\psi$. This is because only the $\Delta$ and $\partial^{2} / \partial t^{2}$ terms in the three respective operators contribute 
in determining this cone. This coincidence can be observed in equations (4.9). These equations give a Klein-Gordon wave solution supported at $c_{o}^{2} t^{2}-\left(z-z^{\prime}\right)^{2}>0$, identical to the support one expects for constant-density solutions.

Let us consider now the integral over $B_{1}$. At this point one should refer to inequalities (4.18) for guidance in selecting a stable initial value problem. As an example, I select the usual geophysical problem by taking

$$
\boldsymbol{\Sigma}=\mathbf{0}
$$

meaning that initial data are specified on a surface $B_{1}$ given by $t=t_{1}$. This problem is stable for $b_{o}^{2}$ positive or negative if the initial data contain only those wavenumbers greater that $\left|b_{o}\right|$ (see equation (4.11)). With the assumptions of inequality (4.39) and equation (4.42), we may simplify our estimate of $E$ to

$$
E \leq \int_{B_{1}} d V E_{v}-\int_{B_{2}} d V E_{v},
$$

which follows from equation (4.38).

We have proven that the energy at any arbitrary time $t_{2}$ is less than or equal to the energy in the initial data, provided there are no sources in $D$. For the case of no sovrces, $E=0$ from equations (4.29) and (4.30). This conservation proof depends on the enforcement of causality, by which the integral over $B$ may be discarded in the energy estimatc. It also depends on selection of a well-posed problem. Without stability, the enersy at time $t_{1}$ is not guaranteed to be bounded and, therefore, the bound on $E_{2}$ in inequality (4.4j) has no real meaning.

I emphasize that these conclusions are independent of whether one does modeling with $p, q$, or $\psi$ fields. We established with equations (4.29) that the energies represented in inequality (4.43) can be formed with any of the three fields. When one transforms the $q$ or $\psi$ field into $p$, the physical field, one finds that acoustic energy has been conserved in the sense of inequality (4.43).

\subsection{The abbreviated variable-density operator}

Let us now analyze the energy relations implied by

$$
\tilde{L}^{(p)} \stackrel{\text { def }}{=} \rho \nabla \cdot\left(\frac{\nabla p}{\rho}\right)-\frac{1}{c^{2}} \frac{\partial^{2} p}{\partial t^{2}}
$$

I have neglected $\nabla \cdot(\nabla \rho / \rho) p$ in the operator, but have not stipulated that this term is zero, or even small. I derive in Appendix A as equation (A-26) the corresponding velocity potential operator,

$$
\tilde{L}^{(\psi)} \stackrel{\text { def }}{=} \frac{1}{\rho} \nabla \cdot(\rho \nabla \psi)+\nabla \cdot\left(\frac{\nabla \rho}{\rho}\right) \psi-\frac{1}{c^{2}} \frac{\partial^{2} \psi}{\partial t^{2}}
$$


In view of equations $(2.2),(2.3)$, and (2.5) one may write the corresponding operator on $q$ as

$$
\tilde{L}^{(q)} \stackrel{\text { def }}{=} \Delta q-\left(\frac{\nabla \rho}{2 \rho} \cdot \frac{\nabla \rho}{2 \rho}\right) q-\frac{1}{c^{2}} \frac{\partial^{2} q}{\partial t^{2}}
$$

Let us again derive Poynting's theorem.

We perform the operations indicated in equations (4.29), but with the new operators, to recognize that

$$
\begin{aligned}
\rho \frac{\partial \psi}{\partial t} \tilde{L}^{(\psi)} \psi & =\frac{p}{\rho} \tilde{L}^{(p)} p^{(t)} \\
& =q \tilde{L}^{(q)} q^{(t)} \\
& =-\nabla \cdot(p v)-\frac{\partial}{\partial t}\left(\frac{1}{2} \frac{1}{\rho c^{2}} p^{2}-\frac{1}{2 \rho} \nabla \cdot\left(\frac{\nabla \rho}{\rho}\right)\left(p^{(t)}\right)^{2}+\frac{1}{2} \rho v \cdot v\right) \\
& =\nabla \cdot P-\frac{\partial}{\partial t}\left(E_{v}-\frac{1}{2 \rho} \nabla \cdot\left(\frac{\nabla \rho}{\rho}\right)\left(p^{(t)}\right)^{2}\right) .
\end{aligned}
$$

These operators spawn a new potential energy term that does not appear in equation (4.23).

$$
\tilde{E}_{v} \stackrel{\text { def }}{=} E_{v}-\frac{1}{2 \rho} \nabla \cdot\left(\frac{\nabla \rho}{\rho}\right)\left(p^{(t)}\right)^{2}
$$

expresses the new energy density $\tilde{E}_{v}$ in terms of the previous energy and that new term.

We smell trouble right away. The additional energy density is negative when the divergence of fractional density is positive. This leaves open the unacceptable possibility of a negative $\tilde{E}_{v}$.

I proceed as before and integrate equations (4.47) over $D$. I designate the initialvalue problem according to equation (4.42) to deal with the integral over $B_{1}$. Moreover, the Poynting vector for these operators is the same as for the previous ones. We may therefore make the estimate of equation (4.37), then insist on causality to simplify the integral over $B$. These steps give

$$
\tilde{E} \leq \int_{B_{1}} d V \tilde{E}_{v}-\int_{B_{2}} d V \tilde{E}_{v}+\int_{B} d V \frac{1}{2 \rho} \nabla \cdot\left(\frac{\nabla \rho}{\rho}\right)\left(p^{(t)}\right)^{2} .
$$

I have also used equations (4.34) to rewrite the remaining integral over $B$ as a volume integral.

As we suspected, the abbreviated variable-density operator has its problems as regards energy conservation. Even without sources, the divergence of fractional density must be zero or negative everywhere to guarantee that the energy at $t_{1}$ bounds the energy at $t_{2}$. This restriction on the medium makes the integrand of the $B$ integral nonpositive, allowing it to be dropped from the estimate.

I interpret inequality (4.49) as proof that one could create density profiles for which the operators of equations (4.44), (4.45), and (4.46) do not conserve acoustic energy. 
This shortcoming may arise for density profiles that abound with large, positive, second derivatives. 


\section{Chapter 5}

\section{A SCALED KLEIN-GORDON WAVE EQUATION}

Now let us make a sharp turn towards scaling of the Klein-Gordon wave equation. In doing so we seek to proceed consistently from the exact wave methods of the preceding material to a companion asymptotic approach. This properly scaled equation will admit an entire spectrum of asymptotic dispersion relations, each corresponding to a different relative choice for the wavelength of density variation. That spectrum of possibilities completes a picture, still developing, of the imprint left by variable density on acoustic traveltimes and fields.

Let us take a Fourier transform over time $t$ in equation (2.2) to obtain

$$
\Delta q(x)-\beta^{2}(x) q+\frac{\omega^{2}}{c^{2}(x)} q=0
$$

with

$$
\beta^{2} \stackrel{\text { def }}{=} \frac{\Delta \sqrt{\rho}}{\sqrt{\rho}} .
$$

The source term in equation (2.2) may be dropped without loss of generality; the ray method, used in obtaining asymptotic solutions, replaces source excitations with equivalent initial data for ordinary differential equations written along the rays. We will prepare equation (5.1) for asymptotic analysis by explicitly representing all length and time scales of its independent variables and coefficients. In the present context these scaling constants carry units of length, inverse time, or their product, and characterize spatial and temporal rates of variation.

We allow that $\beta, \rho$, and $c$ may vary at different rates over space and characterize that variation by introducing new coefficients

$$
\dot{\beta}\left(\frac{2 \pi}{\Lambda_{\beta}} x\right) \stackrel{\text { def }}{=} \beta(x), \quad \tilde{\rho}\left(\frac{2 \pi}{\Lambda_{\rho}} x\right) \stackrel{\text { def }}{=} \rho(x), \quad \tilde{c}\left(\frac{2 \pi}{\Lambda_{c}} x\right) \stackrel{\text { def }}{=} c(x) .
$$

The arguments of these coefficients give $\Lambda_{\beta}, \Lambda_{\rho}$, and $\Lambda_{c}$ the interpretation of characteristic length scales (wavelengths) of the respective physical properties. The $2 \pi$ is not vital. Its presence provides that the argument of the function cycles through $2 \pi$ units every time $|x|$ cycles through a wavelength.

Based on hindsight and some experience with the WKB expansions to come, I also introduce a scaled independent variable,

$$
x=\left(\Lambda_{c} / 2 \pi\right) x_{1}
$$


This is the proper change needed to uncover the asymptotic dispersion relations, alluded to above. Using equations (5.4) and (5.3) in equation (5.1),

$$
\Delta_{1} q\left(\frac{\Lambda_{c}}{2 \pi} x_{1}\right)-\frac{\Lambda_{c}^{2}}{(2 \pi)^{2}} \dot{\beta}^{2}\left(\frac{\Lambda_{c}}{\Lambda_{\beta}} x_{1}\right) q+\frac{\Lambda_{c}^{2}}{(2 \pi)^{2}} \frac{\omega^{2}}{\tilde{c}^{2}\left(x_{1}\right)} q=0,
$$

with

$$
\Delta_{1} \stackrel{\text { def }}{=} \frac{\Lambda_{c}^{2}}{(2 \pi)^{2}} \Delta .
$$

This scaling takes care of the independent variable, making explicit the length scale of derivatives. Next we scale the coefficients in order to represent explicitly their characteristic scales.

I define

$$
\omega=\omega_{o} \tilde{\omega}, \quad \tilde{n}=\frac{c_{o}}{\tilde{c}},
$$

and write from equation (5.5)

$$
\Delta_{1} q\left(\frac{\Lambda_{c}}{2 \pi} x_{1}\right)-\frac{\Lambda_{c}^{2}}{(2 \pi)^{2}} \dot{\beta}^{2}\left(\frac{\Lambda_{c}}{\Lambda_{\beta}} x_{1}\right) q+\frac{\Lambda_{c}^{2}}{\Lambda_{o}^{2}} \tilde{\omega}^{2} \tilde{n}^{2} q=0,
$$

with

$$
\frac{\Lambda_{c}^{2}}{\Lambda_{o}^{2}} \stackrel{\text { def }}{=} \frac{\Lambda_{c}^{2} \omega_{o}^{2}}{(2 \pi)^{2} c_{o}^{2}} .
$$

Symbols $c_{o}$ and $\omega_{o}$ signify a characteristic speed and frequency. We also require an estimate for the magnitude of $\dot{\beta}^{2}$. That is, I wish to scale $\dot{\beta}^{2}$ as

$$
\dot{\beta}^{2}=\left\|\dot{\beta}^{2}\right\| \tilde{\beta}^{2},
$$

where $\tilde{\beta}^{2}$ is defined to be an $O(1)$ quantity like $\tilde{\omega}^{2}$ and $\tilde{n}^{2}$. I will make this estimate in terms of the characteristic wavelength of density.

Employing equations (5.2), (5.3), and (5.4),

$$
\begin{aligned}
\left\|\dot{\beta}^{2}\left(\frac{\Lambda_{c}}{\Lambda_{\beta}} x_{1}\right)\right\| & =\left|\frac{\Delta \sqrt{\rho(x)}}{\sqrt{\rho(x)}}\right| \\
& =\frac{(2 \pi)^{2}}{\Lambda_{c}^{2}}\left|\frac{\Delta_{1} \sqrt{\tilde{\rho}\left(\frac{\Lambda_{c}}{\Lambda_{\rho}} x_{1}\right)}}{\sqrt{\tilde{\rho}\left(\frac{\Lambda_{c} x_{1}}{\Lambda_{\rho}}\right)}}\right| \\
& =\frac{(2 \pi)^{2}}{\Lambda_{c}^{2}} \frac{\Lambda_{c}^{2}}{\Lambda_{\rho}^{2}} .
\end{aligned}
$$

The last line follows because the Laplacian operator that remains-when $\Delta_{1}$ acts-is a Laplacian with respect to $\Lambda_{c} / \Lambda_{\rho} x_{1}$. Thus, the remaining relative curvature term is an 
$O(1)$ quantity, $\tilde{\beta}^{2}$. I substitute this estimate for $\left|\dot{\beta}^{2}\right|$ into equation (5.7), giving

$$
\Delta_{1} q-\Lambda_{c}^{2}\left[\frac{1}{\Lambda_{\rho}^{2}} \tilde{\beta}^{2}\left(\frac{\Lambda_{c}}{\Lambda_{\beta}} x_{1}\right)-\frac{1}{\Lambda_{o}^{2}} \tilde{\omega}^{2} \tilde{n}^{2}\left(x_{1}\right)\right] q\left(\frac{\Lambda_{c}}{2 x} x_{1}\right)=0 .
$$

This wave equation, though scaled, is exact. Formally, all asymptotic analysis of the Klein-Gordon equation should start from equation (5.11).

What have we accomplished through scaling a wave equation? It is well known that finite difference modeling with equation (1.1) simulates wavefield dispersion associated with rapid variation in medium properties. Asymptotic ray solutions must be derived from scaled equations, like equation $(5.11)$, to account for rapid $\left(\Lambda_{\rho}=\Lambda_{0}\right)$ density variation.

Finite differencing accounts automatically for large or small time and length scales. Those scales are implicit in the dimensional operators and coefficients of equations (1.1) and (5.1). On the other hand an analytical, asymptotic treatment relies on the analyst to identify a balance of terms, based on selection of the scaling constants. Those scales must be made explicitly available for the choosing. We will discover in what follows that ray analysis, directly on equation (5.1), corresponds implicitly to choosing $\Lambda_{\rho} \gg \Lambda_{o}$. Implicitly, the density and speed functions are assumed to vary at the same (slow) rate over space. 


\section{Chapter 6}

\section{RAY ANALYSIS}

I will unveil through equation (5.11) a hierarchy of eikonal and transport equations as the ray theoretical analogs of the preceding wave theory. The classical eikonal and transport results (e.g., Cerveny et al., 1977 and Bleistein, 1984) occupy one position in the hierarchy - and thus are subsumed by this approach. This pair of equations provides analytical means for calculating traveltime and amplitude, respectively. For this reason, the eikonal equation may be thought of as an "asymptotic dispersion relation".

We will discover that rapid density variation influences traveltime through the appearance of the acoustic dispersion coefficient in a new eikonal equation. The KleinGordon wave theory gave the same prediction, yet through expressions for group or phase velocities (equations (2.15) and (2.16)). Slow variation belongs with the transport equation, which governs ray amplitudes. Figure 6.1 summarizes qualitatively these coming results.

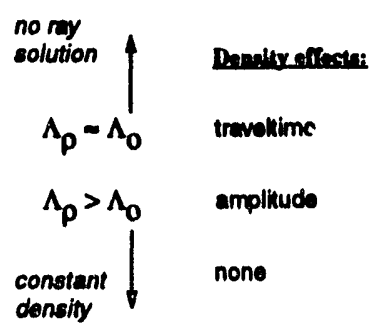

Fig. 6.1. A summary of ray-theoretical results for a variable-density, variable-speed acoustic medium. Density variation on the scale of a seismic wavelength $\Lambda_{o}$ alters the effective slowness of a medium, and thus perturbs traveltime through that medium. Further down in the continuum, where the wavelength $\Lambda_{\rho}$ of density exceeds $\Lambda_{o}$, ray amplitudes are perturbed instead.

We will try the WKB ansatz (Bleistein, 1984),

$$
q\left(\frac{\Lambda_{c}}{2 \pi} x_{1} ; \frac{\Lambda_{c}}{\Lambda_{o}}\right) \sim A\left(\frac{\Lambda_{c}}{2 \pi} x_{1} ; \frac{\Lambda_{c}}{\Lambda_{o}}\right) e^{i \frac{\Lambda_{c}}{\Lambda_{0}} \tau\left(\frac{\Lambda_{c}}{2 \pi} x_{1}\right)}
$$

with $A$ having the formal expansion

$$
A=A_{0}+\frac{A_{1}}{i \Lambda_{c} / \Lambda_{o}}+\frac{A_{2}}{\left(i \Lambda_{c} / \Lambda_{o}\right)^{2}}+\ldots
$$


The character $A$ symbolizes the full amplitude, $A_{0}$ the leading order amplitude, etc. The character $\tilde{\tau}$ is a (dimensionless) traveltime, the conjugate variable of the expansion parameter-dimensionless frequency $\Lambda_{c} / \Lambda_{0}$.

Indorad, we observe that the coefficient $\tilde{\beta}^{2}$ of equation (5.11) depends in part on the expansion parameter. This dependence is unacceptable, because the value of $\Lambda_{c} / \Lambda_{o}$ is arbitrary. Apparently, $\Lambda_{c} / \Lambda_{o}$ must be large for a valid truncated, asymptotic expansion of the solution $q$.

To impart an acceptable meaning to the argument of $\tilde{\beta}^{2}$ I require that $\Lambda_{\beta}=\Lambda_{c}$. This restriction on $\Lambda_{\beta}$ limits the class of media which can be considered, and still obtain a valid asymptotic solution. Specifically, the dispersion coefficient must vary at the same (slow) rate over space as the speed coefficient. In practice, $\Lambda_{\beta}$ and $\Lambda_{c}$ must be at least $3 \Lambda_{o}$ according to Bleistein (1984). Please note, however, that slowly varying $\tilde{\beta}$ does not imply slowly varying $\rho$. In fact, we determined above that a constant dispersion coefficient corresponds tc rapid density variation, when that constant is large.

Setting $\Lambda_{\beta}=\Lambda_{c}$ and inserting the ansatz into equation (5.11) gives

$$
\begin{gathered}
-\frac{\Lambda_{c}^{2}}{\Lambda_{o}^{2}}\left[\nabla_{1} \tilde{\tau} \cdot \nabla_{1} \tilde{\tau}-\tilde{\omega}^{2} \tilde{n}^{2}\left(x_{1}\right)\right] A-\frac{\Lambda_{c}^{2}}{\Lambda_{\rho}^{2}} \tilde{\beta}^{2}\left(x_{1}\right) A \\
+i \frac{\Lambda_{c}}{\Lambda_{o}}\left[2 \nabla_{1} \tilde{\tau} \cdot \nabla_{1} A+A \Delta_{1} \tilde{\tau}\right]+\Delta_{1} A \sim 0
\end{gathered}
$$

We see from equation (6.3) that-through our efforts to scale the wave equation-we have built in the freedom to weight variously the ratio of $\Lambda_{c}$ to $\Lambda_{\rho}$ in an asymptotic balance of terms. That is to say, we are free to choose a wavelength for density variation relative to speed variation.

A continuum of possible ratios $\Lambda_{c} / \Lambda_{\rho}$ is available. However, inspection of equation (6.3) indicates $\Lambda_{c} / \Lambda_{\rho}=\left(\Lambda_{c} / \Lambda_{o}\right)^{j}, j=\ldots,-1,0,1, \ldots$, are positions in that continuum where fundamental reordering of terms occurs. It is at those positions where the $\Lambda_{c} / \Lambda_{\rho}$ term exactly matches, surpasses, or recedes from the other terms with regard to their ordering in $\Lambda_{c} / \Lambda_{o}$, the expansion parameter. I discuss below the two most interesting cases, $\Lambda_{c} / \Lambda_{\rho}=\left(\Lambda_{c} / \Lambda_{o}\right)^{0}$ and $\Lambda_{c} / \Lambda_{\rho}=\left(\Lambda_{c} / \Lambda_{o}\right)^{1}$. From those one can characterize generally the nature of rayfields over the entire spectrum of values for $\Lambda_{c} / \Lambda_{\rho}$.

\subsection{Slow density variation}

We choose $\Lambda_{c} / \Lambda_{\rho}=1$ in equation (6.3) and, consistent with the asymptotic expansion of equation $(6.1)$, take $\Lambda_{c} / \Lambda_{o}$ large enough (by taking $\omega_{o}$ and $\Lambda_{c}$ large enough) such that

$$
\nabla_{1} \tilde{\tau} \cdot \nabla_{1} \tilde{\tau}=\tilde{\omega}^{2} \tilde{n}^{2}\left(x_{1}\right)
$$


By reverting to dimensional coordinates and variables one identifies equation (6.4) as the familiar eikonal equation. Dimensional time $\tau$ is

$$
\tau \stackrel{\text { def }}{=} \frac{\Lambda_{c}}{2 \pi c_{o} \tilde{\omega}} \tilde{\tau}
$$

Refer back to equations (5.3), (5.4), and (5.6) to obtain the dimensional analog

$$
\nabla \tau \cdot \nabla \tau=\frac{1}{c^{2}(\boldsymbol{x})}
$$

Thus, when density varies at the same slow spatial rate that large $\Lambda_{c} / \Lambda_{o}$ requires of the speed coefficient, traveltime is governed by the classical eikonal equation, equation (6.6). Bleistein (1984) demonstrates that one may also obtain equation (6.6) through asymptotic analysis directly on unscaled wave equations, taking $\omega$ to be the expansion parameter. I conclude, by comparison with our result from a scaled equation, that the direct approach corresponds implicitly to choosing $\Lambda_{\rho} \geq \Lambda_{c}$.

Similar analyses give the same eikonal equation and

$$
2 \nabla \tau \cdot \nabla A_{(0)}+A_{(0)} \Delta \tau=0 \quad \forall \Lambda_{c} / \Lambda_{\rho}=\left(\Lambda_{c} / \Lambda_{o}\right)^{j}, j \leq 0 .
$$

This leading order transport result is identical to the one for constant-density media (Bleistein, 1984). Thus, the case $\Lambda_{\rho}=\Lambda_{c}$ serves as a transition point $(j=0)$ for variabledensity acoustic media-below which $(j<0)$ rays propagate as if in a constant-density medium.

\subsection{Rapid density variation}

The choice $\Lambda_{\rho}=\Lambda_{o}$ corresponds, by definition, to a situation where density varies on the same scale as a reference wavelength. This medium is thinbedded with respect to density. Equation (6.3) gives, by dominant balance in powers of $\Lambda_{c} / \Lambda_{o}$,

$$
\begin{aligned}
\nabla \tau \cdot \nabla \tau & =\frac{1}{c^{2}(x)}-\frac{1}{\omega^{2}} \frac{b^{2}(x)}{c^{2}(x)} \\
& =\frac{1}{c^{2}(x)}-\frac{1}{\omega^{2}}\left(\frac{\Delta \sqrt{\rho(x)}}{\sqrt{\rho(x)}}\right) .
\end{aligned}
$$

(I have used equations (5.3), (5.4), (5.6), (5.9), and (6.5) for dimensionalization.)

This new eikonal equation provides the basis for predicting a rayfield traveltime distortion due to density gradients, distinct from the influence exerted through $c$. Moreover, equation (6.8) shows traveltime $\tau$ to vary not only with space but also with frequency, as it should in a dispersive medium. The effective slowness of the medium is altered by frequency and by derivatives of density.

Define the wavenumber $\tilde{k} \stackrel{\text { def }}{=}|\nabla \tau| \omega$ to see that equation (6.8) is the asymptotic 
equivalent of a Klein-Gordon dispersion relation. With that definition one obtains

$$
\tilde{k}^{2}=\frac{\omega^{2}}{c^{2}(\boldsymbol{x})}-\frac{b^{2}(\boldsymbol{x})}{c^{2}(\boldsymbol{x})},
$$

a form similar to that of equation (2.14). Let us compare the two.

The ray-theoretical result in equation (6.9) permits speed and dispersion functions that vary slowly with space, unlike equation (2.14). That generality was bought at the price of requiring, effectively, that $\omega$ be large. On the other hand, the wave-theoretical result in equation (2.14) is val ? for all $\omega$. Differences notwithstanding, these dispersion relations are identical when $b$ and $c$ are constant.

We complete the ray investigation by identifying $\Lambda_{\rho}=\Lambda_{o}$ as another transition position $(j=1)$. Equation (6.3) requires the trivial solution $A \sim 0$ for all $\Lambda_{c} / \Lambda_{\rho}=$ $\left(\Lambda_{c} / \Lambda_{o}\right)^{j}, j>1$. Apparently, waves of the type proposed in equation (6.1) cannot propagate in a medium where density varies still more rapidly.

Let us recount the conditions under which the results of this section hold. To obtain valid ray solutions we require that $\Lambda_{\beta}=\Lambda_{c} \geq 3 \Lambda_{o}$ and $\Lambda_{\rho} \geq \Lambda_{o}$. These conditions quantify the statements of Figure 6.1. 


\section{Chapter 7}

\section{SCATTERING AT AN INTERFACE}

A Klein-Gordon theory for acoustics would not be complete without considering the physics required of the $q$ field at a discontinuity in physical properties. Case in point, I have shown already that the dispersion coefficient cannot be constant for an indefinite region over space without violating physical limits on density. Klein-Gordon constant-coefficient modeling therefore requires conditions that properly continue fields across interfaces, from one medium to the next.

The goal will be to obtain conditions on $q$ that impart the proper physics to the corresponding physical field $(p)$ at an interface. In this case the proper physics is deemed to be conservation of mass and momentum. Ultimately, those conditions will expose a new rule for how $p$ or $q$ waves and rays bend at an interface between two variable-density, variable-velocity media.

\subsection{Interface conditions}

Consider scattering of an acoustic wavefield from a planar interface separating two continuous media. This type of interface may be parameterized by its unit normal $\hat{n}$ and the signed distance $d$ along the normal to the origin of the coordinate system. All points $x$ on the interface satisfy

$$
\hat{\boldsymbol{n}} \cdot \boldsymbol{x}=d .
$$

This interface geometry is drawn in Figure 7.1.

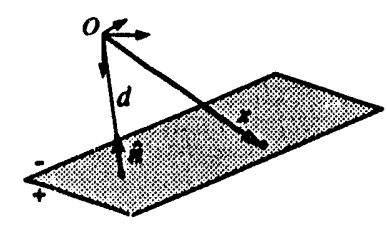

FIG. 7.1. Parameterization of a planar scattering surface. The normal $\hat{\boldsymbol{n}}$ to the plane and distance $d$, measured along a normal through the origin, define a planar surface on which $\hat{\boldsymbol{n}} \cdot \boldsymbol{x}=d$.

Taking a hint from Stakgold (1979), we require that the total acoustic field $p$ satisfies

$$
\Delta p(\boldsymbol{x}, t)-\nabla \cdot\left[\frac{\nabla \rho}{\rho(\boldsymbol{x})} p\right]-\frac{1}{c^{2}(\boldsymbol{x})} \frac{\partial^{2} p}{\partial t^{2}}=0
$$


with

$$
\begin{aligned}
\nabla \rho & =\nabla \rho_{-}+H(\xi)\left[\nabla \rho_{+}-\nabla \rho_{-}\right] \\
\frac{1}{\rho} & =\frac{1}{\rho_{-}}+H(\xi)\left[\frac{1}{\rho_{+}}-\frac{1}{\rho_{-}}\right] \\
\frac{1}{c^{2}} & =\frac{1}{c_{-}^{2}}+H(\xi)\left[\frac{1}{c_{+}^{2}}-\frac{1}{c_{-}^{2}}\right]
\end{aligned}
$$

The operator form in equation (7.2) is equivalent to that in equation (1.1), and is derived as equation $(A-18)$ in Appendix $A$. The Heaviside distribution $H$ is defined as before, in equation (4.3), making no attempt to assign a value at $\xi=0$. I wish the argument $\xi$ to represent a signed distance from the interface, along its normal. Hence, from equation (7.1)

$$
\xi \stackrel{\text { def }}{=} \hat{\boldsymbol{n}} \cdot \boldsymbol{x}-\boldsymbol{d}
$$

Our task is to derive conditions that ensure equation (7.2) is satisfied at all field points, including those on the interface $\xi=0$. Many authors (Aki and Richards, 1980; Auld, 1990) approach this derivation by integrating equation (7.2) over a volume that includes a portion of the interface. Those terms in the integrand which are an exact divergence are evaluated at the surface of the volume, thus giving an expression on the interface as the volume tends toward vanishing. The form of the wave equation chosen for equation (7.2) is suited ideally for that tactic. I shall take, however, a different tack, seeing with hindsight a course that involves less subtlety.

I begin by constructing the total field $p$ as

$$
p=p_{-}+H(\xi)\left(p_{+}-p_{-}\right)
$$

where

$$
\Delta p_{-}-\nabla \cdot\left[\frac{\nabla \rho_{-}}{\rho_{-}} p_{-}\right]-\frac{1}{c_{-}^{2}} \frac{\partial^{2} p_{-}}{\partial t^{2}}=0
$$

and

$$
\Delta p_{+}-\nabla \cdot\left[\frac{\nabla \rho_{+}}{\rho_{+}} p_{+}\right]-\frac{1}{c_{+}^{2}} \frac{\partial^{2} p_{+}}{\partial t^{2}}=0
$$

We shall discover consequently rhat

$$
\left(p_{-}\right)_{\xi=0}=\left(p_{+}\right)_{\xi=0}, \quad\left(\frac{\partial p_{-}}{\partial n}-\frac{1}{\rho_{-}} \frac{\partial \rho_{-}}{\partial n} p_{-}\right)_{\xi=0}=\left(\frac{\partial p_{+}}{\partial n}-\frac{1}{\rho_{+}} \frac{\partial \rho_{+}}{\partial n} p_{+}\right)_{\xi=0}
$$

The definitions

$$
n \stackrel{\text { def }}{=} \hat{\boldsymbol{n}} \cdot \boldsymbol{x}, \quad \partial / \partial n \stackrel{\text { def }}{=} \hat{\boldsymbol{n}} \cdot \nabla,
$$

make explicit the meaning of the derivatives in equations (7.8).

The construction in equation (7.5) makes the proper a priori postulation about the solution. In the limit as distance from the interface vanishes via the " - "medium, 
$p \rightarrow p_{-}$. Likewise, $p \rightarrow p_{+}$as $\xi \rightarrow 0_{+}$.

Let us substitute equations (7.5) and (7.3) into equation (7.2). We need

$$
\begin{aligned}
\nabla p & =\nabla p_{-}+\nabla\left[H(\xi)\left(p_{+}-p_{-}\right)\right] \\
& =\nabla p_{-}+H(\xi) \nabla\left(p_{+}-p_{-}\right)+\delta(\xi)\left(p_{+}-p_{-}\right) \hat{\boldsymbol{n}},
\end{aligned}
$$

taking $\delta$ to be the Dirac delta function and employing the chain rule,

$$
\frac{\partial}{\partial x_{j}}=\frac{\partial \xi}{\partial x_{j}} \frac{\partial}{\partial \xi}=n_{j} \frac{\partial}{\partial \xi}
$$

and equation (7.4) for differentiation. Using the expansion of $\nabla p$ and

$$
\Delta\left[H(\xi)\left(p_{+}-p_{-}\right)\right]=H(\xi) \Delta\left(p_{+}-p_{-}\right)+2 \delta(\xi) \frac{\partial}{\partial n}\left(p_{+}-p_{-}\right)+\delta^{\prime}(\xi)\left(p_{+}-p_{-}\right)
$$

one obtains

$$
\begin{aligned}
& {\left[\Delta p_{-}-\nabla \cdot\left(\frac{\nabla \rho_{-}}{\rho_{-}} p_{-}\right)-\frac{1}{c_{-}^{2}} \frac{\partial^{2} p_{-}}{\partial t^{2}}\right]} \\
& -\left[\Delta p_{-}-\nabla \cdot\left(\frac{\nabla \rho_{-}}{\rho_{-}} p_{-}\right)-\frac{1}{c_{-}^{2}} \frac{\partial^{2} p_{-}}{\partial t^{2}}\right] H(\xi) \\
& +\left[\Delta p_{+}-\nabla \cdot\left(\frac{\nabla \rho}{\rho} p_{+}\right)-\frac{1}{c_{+}^{2}} \frac{\partial^{2} p_{+}}{\partial t^{2}}\right] H(\xi) \\
& +\left[2 \frac{\partial}{\partial n}\left(p_{+}-p_{-}\right)-\frac{p_{+}}{\rho_{+}} \frac{\partial \rho_{+}}{\partial n}+\frac{p_{-}}{\rho_{-}} \frac{\partial \rho_{-}}{\partial n}\right] \delta(\xi)+\left[p_{+}-p_{-}\right] \delta^{\prime}(\xi)=0 .
\end{aligned}
$$

The " $\prime$ " on the delta function indicates formally the operation $\partial / \partial \xi$. This derivative is just $\partial / \partial n$ since

$$
\frac{\partial}{\partial \xi}=\frac{\partial n}{\partial \xi} \frac{\partial}{\partial n}=\frac{\partial}{\partial n}
$$

from equations (7.4) and (7.9). The first three terms of equation (7.10) are zero for all $\boldsymbol{x}$, including those $\boldsymbol{x}$ such that $\xi=0$, as I show now.

The first three terms sum to zero, from equations (7.6) and (4.3), for all space such that $\xi \leq 0 \ldots$. For $\xi \geq 0_{+}$the Heaviside distribution has support. Yet, even though $p_{-}$ does not satisfy a wave equation in the " + " region, the first two (nonzero) terms cancel exactly. The remaining third term of equation (7.10) produces zero contribution in the " + " region because $p_{+}$is a wave solution there (equation (7.7)).

We see then that only the $\delta$ and $\delta^{\prime}$ terms in equation (7.10), supported exclusively on the interface, determine conditions at the interface. Assuming that $p_{+}-p_{-}$is bounded 
as $|\xi| \rightarrow \infty$ and continuous as $\xi \rightarrow 0$, we may rewrite the $\delta^{\prime}$ term as

$$
\delta^{\prime}\left(p_{+}-p_{-}\right)=-\delta \frac{\partial}{\partial n}\left(p_{+}-p_{-}\right)
$$

One obtains the second condition of equations (7.8) as the consequence of placing this distributional equality in equation (7.10). Our assumption of continuous $p_{+}-p_{-}$sets the first condition. Since $p_{+}$and $p_{-}$represent perturbations from a background, continuity of their difference must mean that the field is continuous at the interface.

To summarize our progress, the continuity constraints of equations (7.8) ensure that-for two juxtaposed variable-density media- $p$ satisfies the wave equation at the intervening interface. Equations (7.8) reduce to their constant-density counterparts (Bleistein (1984)) if $\partial \rho / \partial n$ is zero.

The requisite conditions at an acoustic interface mny also be stated as

$$
\left(p_{-}\right)_{\xi=0}=\left(p_{+}\right)_{\xi=0}, \quad\left(\rho_{-} v_{-} \cdot \hat{n}\right)_{\xi=0}=\left(\rho_{+} v_{+} \cdot \hat{n}\right)_{\xi=0} .
$$

This pair of stipulations can be derived, respectively, from the field equations (A-16) and (A-17). Equations (7.11) do not afford a convenient formulation for modeling, as they involve calculations of both $p$ and $v$ at an interface, unlike equations (7.8).

Importantly however, a continuity derivation from equation (A-17) identifies continuity of pressure as a statement of conservation of momentum at an interface. Similarly, mass conservation and the equation of state (equation (A-16)) demand a continuous normal component of momentum density $(\rho v)$ at an interface. Comparing equations (7.8) with equations (7.11) we may conclude that continuous $\partial p / \partial n-(1 / \rho)(\partial \rho / \partial n) p$ enforces these latter two principles between variable-density media. The acoustic wave equation embodies all of these physical prinsiples; both continuity conditions may therefore be wrested from equation (7.2) alone.

We wish to do scattering problems in the $q$ variable, as well as $p$. With reference to equation (2.1) I adopt

$$
\begin{aligned}
\left(q_{-} \sqrt{\rho_{-}}\right)_{\xi=0} & =\left(q_{+} \sqrt{\rho_{+}}\right)_{\xi=0}, \\
\left(\frac{\partial}{\partial n}\left(q_{-} \sqrt{\rho_{-}}\right)-\frac{1}{\sqrt{\rho_{-}}} \frac{\partial \rho_{-}}{\partial n} q\right)_{\xi=0} & =\left(\frac{\partial}{\partial n}\left(q_{+} \sqrt{\rho_{+}}\right)-\frac{1}{\sqrt{\rho_{+}}} \frac{\partial \rho_{+}}{\partial n} q\right)_{\xi=0},
\end{aligned}
$$

as the continuity conditions on $q$. Significantly, this convention preserves continuity of the physical quantities at the interface. That is to say, equations (7.12) compel KleinGordon waves to conserve mass and acoustic momentum and honor the acoustic equation of state at an interface.

Omission of that wave equation term-involving the divergence of fractional densityproduces hybrid interface conditions. These conditions are intermediate in complexity 
between equations (7.8) and the constant-density conditions. Specifically, taking

$$
\rho \nabla \cdot\left[\frac{\nabla p}{\rho}\right]-\frac{1}{c^{2}} \frac{\partial^{2} p}{\partial t^{2}}=0
$$

as the wave equation,

$$
\left(p_{-}\right)_{\xi=0}=\left(p_{+}\right)_{\xi=0}, \quad\left(\frac{1}{\rho_{+}} \frac{\partial p_{+}}{\partial n}\right)_{\xi=0}=\left(\frac{1}{\rho_{-}} \frac{\partial p_{-}}{\partial n}\right)_{\xi=0},
$$

are the resulting interface conditions.

The latter condition of equations (7.14) may also be obtained by assuming $\boldsymbol{v} \cdot \boldsymbol{n}$ is continuous (Brekhovskikh, 1960), allowing $\rho$ to jump at the interface, then neglecting the normal derivative of $\rho$. One must use the relations between $\boldsymbol{p}, \boldsymbol{v}$, and $\psi$ (equations (4.19) and (A-20)) to confirm this. This density treatment is inconsistent, in that equations (7.11) expose continuous $\boldsymbol{v} \cdot \boldsymbol{n}$ as a constant-density assumption, permitting no jump in $\rho$ at an interface. This approach to variable-density problems is analogous to how one derives equation (7.13) from first principles (DeSanto, 1992). A select mixture of constant- and variable-density assumptions are employed.

\subsection{Plane-wave scattering}

Let us derive coefficients, from the continuity conditions, that describe scattering of Klein-Gordon plane waves from an interface like the one in Figure 7.1. Besides their value for computation, the interpretation of these coefficients provides insight into the scattering process that cannot be ascertained directly from the continuity conditions.

For example, the scattering process-as is propagation-is frequency-dependent in a variable-density acoustic medium. Moreover, the operators in equations (1.1) or (7.2) allow for large density gradients on either side of a scattering interface, but do not admit jumps in density itself at the interface. On the other hand, the shortened operator in equation (7.13) permits density to jump at an interface, but requires that $\nabla \cdot(\nabla \rho / \rho)$ be negligible on either side.

I interpret these limitations in the variable-density operators as the inevitable result of neglecting the force of gravity. I determine in Appendix A that, absent this force, the initial state of the medium must be dynamir, rather that static, equilibrium. This compromise is required in order to honor the variable-density ramifications of the acoustic equation of state.

As with pressure, one may prescribe $q$ as

$$
q=q_{-}+H(\xi)\left(q_{+}-q_{-}\right)
$$

to acknowledge the influence on the field of an interface between two media. Two continuity conditions on $q$ at an interface provide means for calculating two new waves generated 
by scattering. Based on physical experience one takes

$$
\begin{aligned}
& q_{-}=q_{I}+q_{R}, \\
& q_{+}=q_{T},
\end{aligned}
$$

thinking of reflected and transmitted waves, in addition to the incident wave. Figure 7.2 depicts this notion.

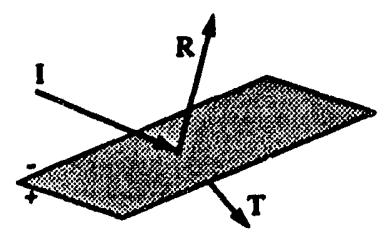

Fig. 7.2. Scattering at an interface between two acoustic media. Two independent interface conditions require that an incident (I) wave or ray scatters into reflected (R) and transmitted $(\mathrm{T})$ waves or rays.

Notation in place, we first apply equations (7.12) and (7.15) to obtain to

$$
\begin{gathered}
{\left[\left(q_{I}+q_{R}\right) \sqrt{\rho_{-}}\right]_{\xi=0}=\left[q_{T} \sqrt{\rho_{+}}\right]_{\xi=0},} \\
\frac{\partial}{\partial n}\left[\left(q_{I}+q_{R}\right) \sqrt{\rho_{-}}\right]_{\xi=0}=\frac{\partial}{\partial n}\left[q_{T} \sqrt{\rho_{+}}\right]_{\xi=0} .
\end{gathered}
$$

Equations (7.16) and (7.17) describe the scattering process implied by the operator in equation (7.2). We next assume solutions for the incident and scattered waves,

$$
\begin{aligned}
& q_{I}=A_{I} e^{i\left[k_{-}\left(t_{2} \sin \theta_{I}+\xi \cos \theta_{I}\right)-\omega t\right]}, \\
& q_{R}=A_{R} e^{i\left[k_{-}\left(t_{2} \sin \theta_{R}-\xi \cos \theta_{I}\right)-\omega t\right]}, \\
& q_{T}=A_{T} e^{i\left[k_{+}\left(t_{2} \sin \theta_{T}+\xi \cos \theta_{T}\right)-\omega t\right]} .
\end{aligned}
$$

In these solutions, $t_{2}$ is a tangent direction in the scattering plane and $\xi$ is the coordinate normal to the plane. The subscripted $\theta$-variables represent incident and scattering angles at the interface, given by $\xi=0$. See Figure $7.3 \mathrm{~d}$ for a drawing of this geometry.

These are valid solutions to the constant-coefficient, Klein-Gordon wave equation if

$$
k=\omega \sqrt{\frac{1}{c_{o}^{2}}-\frac{1}{\omega^{2}}\left(\frac{\Delta \sqrt{\rho}}{\sqrt{\rho}}\right)_{0}} .
$$


Furthermore, from equation (7.16), if these solutions are evaluated at $\xi=0$ and time $t$ is held constant,

$$
k_{-} \sin \theta_{I}=k_{-} \sin \theta_{R}=k_{+} \sin \theta_{T} .
$$

We thus obtain laws of refleciion and refraction,

$$
\begin{aligned}
\theta_{I} & =\theta_{R}, \\
\sin \theta_{I} \sqrt{\frac{1}{c_{-}^{2}}-\frac{1}{\omega^{2}}\left(\frac{\Delta \sqrt{\rho}}{\sqrt{\rho}}\right)_{-}} & =\sin \theta_{T} \sqrt{\frac{1}{c_{+}^{2}}-\frac{1}{\omega^{2}}\left(\frac{\Delta \sqrt{\rho}}{\sqrt{\rho}}\right)_{+}} .
\end{aligned}
$$

Equation (7.24) states Snell's Law with new generality. Plane-wave refraction at an interface depends on the dispersion coefficient as well as the speed of acoustic materials. For constant densities, or large enough frequency, this general result reduces to Snell's classical result.

We now pursue the controls on scattered amplitudes. Using equations (7.22) and substitution of the constant-coefficient solutions into equations (7.16) and (7.17),

$$
\begin{gathered}
\sqrt{\rho_{-}} k_{-} \cos \theta_{I}(1-R)+i \frac{1}{2} \frac{1}{\sqrt{\rho_{-}}} \frac{\partial \rho_{-}}{\partial n}(1+R)= \\
\sqrt{\rho_{+}} k_{+} \cos \theta_{T} T+i \frac{1}{2} \frac{1}{\sqrt{\rho_{+}}} \frac{\partial \rho_{+}}{\partial n} T
\end{gathered}
$$

and

$$
1+R=\frac{\sqrt{\rho_{+}}}{\sqrt{\rho_{-}}} T
$$

at the scattering interface. Here, the reflection and transmission coeffcients, $R$ and $T$, are

$$
R \stackrel{\text { def }}{=} \frac{A_{R}}{A_{I}}, \quad T \stackrel{\text { def }}{=} \frac{A_{T}}{A_{I}} .
$$

In solving equations (7.25) and (7.26) for $R$, I find that

$$
\begin{gathered}
R=\frac{\left(k_{-} \cos \theta_{I}-k_{+} \cos \theta_{T}\right)\left(k_{-} \cos \theta_{I}+k_{+} \cos \theta_{T}\right)-\frac{1}{4}(\Delta \gamma)^{2}}{\left(k_{-} \cos \theta_{I}+k_{+} \cos \theta_{T}\right)^{2}+\frac{1}{4}(\Delta \gamma)^{2}}-i \Im R, \\
\Im R=\frac{\Delta \gamma k_{-} \cos \theta_{I}}{\left(k_{-} \cos \theta_{I}+k_{+} \cos \theta_{T}\right)^{2}+\frac{1}{4}(\Delta \gamma)^{2}}
\end{gathered}
$$

The difference $\Delta \gamma$,

$$
\Delta \gamma \stackrel{\text { def }}{=} \frac{1}{\rho_{+}} \frac{\partial \rho_{+}}{\partial n}-\frac{1}{\rho_{-}} \frac{\partial \rho_{-}}{\partial n},
$$

indicates the jump in fractional density at the interface.

I note four points about this reflection coefficient. First, it is complex, indicating a more complicated interaction of wave with interface than that which occurs at a den- 
sity jump between two constant-density media (DeSanto, 1992). We will write down that (real) result below. Secondly, recalling equation (7.21), reflection amplitudes are frequency-dependent. In addition, $\Im R=0$ if fractional density is continuous at the interface-as it is in the models of Figure 1.2.

I shall make the fourth point through the example of Figure 1.2. I will conclude that the differential operator in equation (7.2) precludes the possibility of discontinuous density profiles, though discontinuous dispersion and fractional densities are allowed.

For any single model in this collection of density models the fractional density and dispersion coefficient do not vary from one layer to the next. I showed above that exponential density profiles maintain this simple relation between fractional density and the dispersion coefficient. For no jump in fractional density, equation (7.28) simplifies to

$$
R=\frac{k_{-} \cos \theta_{I}-k_{+} \cos \theta_{T}}{k_{-} \cos \theta_{I}+k_{+} \cos \theta_{T}}
$$

Since speed $c$ and $\Delta \sqrt{\rho} / \sqrt{\rho}$ are constants here, this result would seem to claim that the models in Figure 1.2 have no reflectivity at normal incidence! The data in Figure 1.1which do not depend on this result-discount that possibility. Moreover, models having a jump in density between two constant-density, constant-speed media give rise to the same dilemma for equation (7.28).

I interpret equation (7.31) to mean that the physics imposed by equations (1.1) or (7.2) preclude discontinuous density profiles. Indeed, we found in the derivation of equation (1.1), in Appendix $A$, that this differential equation assumes a background medium in hydrodynamic - rather than hydrostatic-equilibrium. Abrupt changes in density are therefore disallowed. We will find, below, that the differential operator in equation (7.13) admits jumps in density. We know already, however, that this flexibility is gained at the price of requiring small density gradients.

One may also solve equations (7.16) and (7.17) for the transmission coefficient to obtain

$$
T=\frac{2 k_{-} \cos \theta_{I}\left(k_{-} \cos \theta_{I}+k_{+} \cos \theta_{T}\right)}{\left(k_{-} \cos \theta_{I}+k_{+} \cos \theta_{T}\right)^{2}+\frac{1}{4}(\Delta \gamma)^{2}}-i \Im R .
$$

The same four points made for $R$ apply to $T$. In particular, I have required that

$$
\frac{\sqrt{\rho_{-}}}{\sqrt{\rho_{+}}}=1
$$

in equation (7.26), to obtain equation (7.32).

The derivation of reflection and transmission coefficients for equation (7.13) follows the same pattern. This derivation again rests on the foundation that equation (7.13) can be written in the Klein-Gordon form, validating equations (7.18), (7.19), and (7.20) as constant-dispersion, constant-speed solutions.

Beginning with the conditions of equations (7.14) and employing the relation $p=$ 
$\sqrt{\rho} q$,

$$
\tilde{R}=\frac{\rho_{+} k_{-} \cos \theta_{I}-\rho_{-} k_{+} \cos \theta_{T}}{\rho_{+} k_{-} \cos \theta_{I}+\rho_{-} k_{+} \cos \theta_{T}}
$$

and

$$
\tilde{T}=\frac{2 \rho_{+} k_{-} \cos \theta_{I}}{\rho_{+} k_{-} \cos \theta_{I}+\rho_{-} k_{+} \cos \theta_{T}},
$$

where

$$
1+\tilde{R}=\frac{\sqrt{\rho_{+}}}{\sqrt{\rho_{-}}} \tilde{T} .
$$

These coefficients are identical in form to those appropriate for scattering between two constant-speed, constant-density media (DeSanto, 1992) - with a jump in both speed and density at the scattering interface. Their substance differs in the expression required for wavenumber $k$.

\subsection{Ray scattering}

In what follows, I develop the results necessary to treat rays at interfaces, in accordance with equation (7.2). We will find these expressions to be in harmony with those for plane-wave scattering, and will gain insight into the importance of the wavelength, $\Lambda_{\rho}$, of the density function. If the density variation is rapid $\left(\Lambda_{\rho} \approx \Lambda_{o}\right)$, then discontinuities in fractional density and the dispersion coefficient scatter acoustic rays. Moreover, I will again make the interpretation that equation (7.2) precludes the possibility of discontinuous density.

This treatment is fashioned after Bleistein (1984). Two essential differences are the use of equations (7.12), rather than continuity conditions for constant-density media, and our new eikonal equation, equation (6.8). The former difference modifies the scattering coefficients, and the latter returns us to a general form of Snell's Law-containing frequency and the dispersion coefficient.

Beginning with equations (7.12), I shall first emphasize development of scattering coefficients that regulate amplitude partitioning of the incident ray into reflected and transmitted rays. It will become apparent that the controls on ray bending must also be investigated in order to express those coefficients entirely in tcrms of the incident ray. That investigation leads to laws of reflection and refraction.

Just as we scaled the Klein-Gordon wave equation, we must also scale the condition on the lower line of equations (7.12). This expression contains derivatives on density, and we require an order estimate of their magnitude in relation to the other terms. In its scaled form, and incorporating equation (7.15),

$$
\begin{gathered}
\sqrt{\tilde{\rho}_{-}} \frac{\partial}{\partial n_{1}}\left(q_{I}+q_{R}\right)-\frac{1}{2} \frac{\Lambda_{c}}{\Lambda_{\rho}} \frac{1}{\sqrt{\tilde{\rho}_{-}}} \frac{\partial \tilde{\rho}_{-}}{\partial\left(\frac{\Lambda_{c}}{\Lambda_{\rho}} n_{1}\right)}\left(q_{I}+q_{R}\right)= \\
\sqrt{\tilde{\rho}_{+}} \frac{\partial q_{T}}{\partial n_{1}}-\frac{1}{2} \frac{\Lambda_{c}}{\Lambda_{\rho}} \frac{1}{\sqrt{\tilde{\rho}_{+}}} \frac{\partial \tilde{\rho}_{+}}{\partial\left(\frac{\Lambda_{c}}{\tilde{\rho}_{\rho}} n_{1}\right)} q_{T} .
\end{gathered}
$$


In this equation, $n_{1}$ is a dimensionless coordinate, normal to the interface, and is analogous to $x_{1}$ in equation (5.4). Equation (5.3) defines $\tilde{\rho}$. The scaled density terms,

$$
\frac{1}{\sqrt{\tilde{\rho}}} \frac{\partial \tilde{\rho}}{\partial\left(\frac{\Lambda_{c}}{\Lambda_{\rho}} n_{1}\right)}
$$

are $O(1)$ quantities. Neglecting this scaling amounts to choosing $\Lambda_{\rho}>>\Lambda_{o}$, and thus from the outset excludes results for $\Lambda_{\rho} \approx \Lambda_{o}$.

As in equation (6.1), I shall substitute WKB series expansions (Bender and Orszag, 1978), in dimensionless variables, into the continuity conditions. That is,

$$
\begin{aligned}
& q_{I} \sim A^{I}\left(\frac{\Lambda_{c}}{2 \pi} x_{1} ; \frac{\Lambda_{c}}{\Lambda_{0}}\right) e^{i \frac{\Lambda_{c}}{\Lambda_{0}} \tilde{\tau}_{I}}, \\
& q_{R} \sim A^{R}\left(\frac{\Lambda_{c}}{2 \pi} x_{1} ; \frac{\Lambda_{c}}{\Lambda_{o}}\right) e^{i \frac{\Lambda_{c}}{\Lambda_{0}} \tilde{\tau}_{R}}, \\
& q_{T} \sim A^{T}\left(\frac{\Lambda_{c}}{2 \pi} x_{1} ; \frac{\Lambda_{c}}{\Lambda_{0}}\right) e^{i \frac{\Lambda_{c}}{\Lambda_{0}} \tilde{T}_{T}},
\end{aligned}
$$

for the incident, reflected, and transmitted fields. Implicit in Figure 7.2, we are interested in relating those fields at the same propagation time and therefore take

$$
\left(\tau_{I}\right)_{\xi=0}=\left(\tau_{R}\right)_{\xi=0}=\left(\tau_{T}\right)_{\xi=0}
$$

When substituted into equations (7.16) and (7.36), equations (7.37) and (7.38) render

$$
\begin{gathered}
{\left[\left(A_{0}^{I}+A_{0}^{R}\right) \sqrt{\rho_{-}}\right]_{\xi=0}=\left[A_{0}^{T} \sqrt{\rho_{+}}\right]_{\xi=0},} \\
{\left[\left(A_{0}^{I} \frac{\partial \tau_{I}}{\partial n}+A_{0}^{R} \frac{\partial \tau_{R}}{\partial n}\right) \sqrt{\rho_{-}}\right]_{\xi=0}=\left[A_{0}^{T} \frac{\partial \tau_{T}}{\partial n} \sqrt{\rho_{+}}\right]_{\xi=0}, \quad\left(\Lambda_{\rho}>>\Lambda_{o}\right),}
\end{gathered}
$$

and

$$
\begin{array}{r}
{\left[\left(A_{0}^{I} \frac{\partial \tau_{I}}{\partial n}+A_{0}^{R} \frac{\partial \tau_{R}}{\partial n}\right) \sqrt{\rho_{-}}+i \frac{1}{2 \omega} \frac{1}{\sqrt{\rho_{-}}} \frac{\partial \rho_{-}}{\partial n}\left(A_{0}^{I}+A_{0}^{R}\right)\right]_{\xi=0}=} \\
{\left[A_{0}^{T} \frac{\partial \tau_{T}}{\partial n} \sqrt{\rho_{+}}+i \frac{1}{2 \omega} \frac{1}{\sqrt{\rho_{+}}} \frac{\partial \rho_{+}}{\partial n} A_{0}^{T}\right]_{\xi=0}, \quad\left(\Lambda_{\rho} \approx \Lambda_{o}\right)}
\end{array}
$$

to leading order in $\omega$. In addition to equations (5.4) and (5.3), I have used equations (5.6), (5.8), and (6.5) to revert to dimensioned coordinates and unscaled variables.

The system of equations (7.39), (7.40), and (7.41) is linear in the unknown quantities 
$A_{0}^{R}$ and $A_{0}^{T}$. Straightforward manipulations of this system give

$$
R_{0} \stackrel{\text { def }}{=} \frac{A_{0}^{R}}{A_{0}^{I}}=\frac{\left[\frac{\partial \tau_{I}}{\partial n}-\frac{\partial \tau_{T}}{\partial n}\right]}{\left[\frac{\partial \tau_{T}}{\partial n}-\frac{\partial \tau_{R}}{\partial n}\right]}, \quad\left(\Lambda_{\rho}>>\Lambda_{o}\right)
$$

or

$$
R_{0}=\frac{\left[\frac{\partial \tau_{I}}{\partial n}-\frac{\partial \tau_{T}}{\partial n}\right]\left[\frac{\partial \tau_{T}}{\partial n}-\frac{\partial \tau_{R}}{\partial n}\right]-\left[\frac{1}{2 \omega} \Delta \gamma\right]^{2}}{\left[\frac{\partial \tau_{T}}{\partial n}-\frac{\partial \tau_{k}}{\partial n}\right]^{2}+\left[\frac{1}{2 \omega} \Delta \gamma\right]^{2}}+i \Im R_{0}, \quad\left(\Lambda_{\rho} \approx \Lambda_{o}\right)
$$

for all $\boldsymbol{x}$ such that $\xi=0$. Here,

$$
\Im R_{0}=\frac{1}{2 \omega} \frac{\Delta \gamma\left[\frac{\partial \tau_{R}}{\partial n}-\frac{\partial \tau_{I}}{\partial n}\right]}{\left[\frac{\partial \tau_{T}}{\partial n}-\frac{\partial \tau_{R}}{\partial n}\right]^{2}+\left[\frac{1}{2 \omega} \Delta \gamma\right]^{2}}
$$

where $\Delta \gamma$ is defined in equation (7.30). Proceeding similarly,

$$
T_{0} \stackrel{\text { def }}{=} \frac{A_{0}^{T}}{A_{0}^{I}}=\frac{\sqrt{\rho_{-}}}{\sqrt{\rho_{+}}} \frac{\left[\frac{\partial \tau_{R}}{\partial n}-\frac{\partial \tau_{I}}{\partial n}\right]}{\left[\frac{\partial \tau_{R}}{\partial n}-\frac{\partial \tau_{T}}{\partial n}\right]}, \quad\left(\Lambda_{\rho}>>\Lambda_{o}\right),
$$

or

$$
T_{0}=\frac{\left[\frac{\partial \tau_{R}}{\partial n}-\frac{\partial \tau_{I}}{\partial n}\right]\left[\frac{\partial \tau_{R}}{\partial n}-\frac{\partial \tau_{T}}{\partial n}\right]}{\left[\frac{\partial \tau_{R}}{\partial n}-\frac{\partial \tau_{T}}{\partial n}\right]^{2}+\left[\frac{1}{2 \omega} \Delta \gamma\right]^{2}}+i \Im R_{0}, \quad\left(\Lambda_{\rho} \approx \Lambda_{o}\right)
$$

The same points made for the plane-wave scattering coefficients are evident for these coefficients, but qualified in terms of the relative wavelength of the density function. For rapid density variation, the scattering coefficients are complex, frequency-dependent quantities. They reduce, however, to real quantities for those special profiles where fractional density does not jump at an interface. Moreover, for those profiles, the coefficients for $\Lambda_{\rho} \approx \Lambda_{o}$ revert to those for $\Lambda_{\rho} \gg \Lambda_{o}$-which do not allow for density jumps at interfaces. The latter expressions are iound in Bleistein (1984), and may be obtained by beginning from the constant-density wave equation. As above, I interpret this observation to mean that equation (7.2) does not admit jumps in density.

Scattering coefficients $R_{0}$ and $T_{0}$ express reflected and transmitted amplitudes rel- 
ative to the amplitude of the incident ray. Notwithstanding, satisfactory completion of our scattering analysis depends on re-expressing $\partial \tau_{R} / \partial n$ and $\partial \tau_{T} / \partial n$, both presumed unknown, in terms of $\partial \tau_{I} / \partial n$.

The simple statement of equations (7.38) in tandem with an eikonal equation will lead us to the desired expressions for reflection and transmission traveltime gradients. First recognize that

$$
\left(\nabla_{t} \tau_{I}\right)_{\xi=0}=\left(\nabla_{t} \tau_{R}\right)_{\xi=0}=\left(\nabla_{t} \tau_{T}\right)_{\xi=0}
$$

from equations (7.38). The symbol $\nabla_{t}$ denotes any gradient acting tangent to the scattering surface given by equation (7.1). One's first inclination may be to represent the geometry of equations (7.47) as in Figure 7.3a, where the restriction on $\nabla_{t}$ is satisfied in the $t_{2}$ direction. However, the azimuthal orientation of $\nabla_{t}$ is arbitrary, requiring that
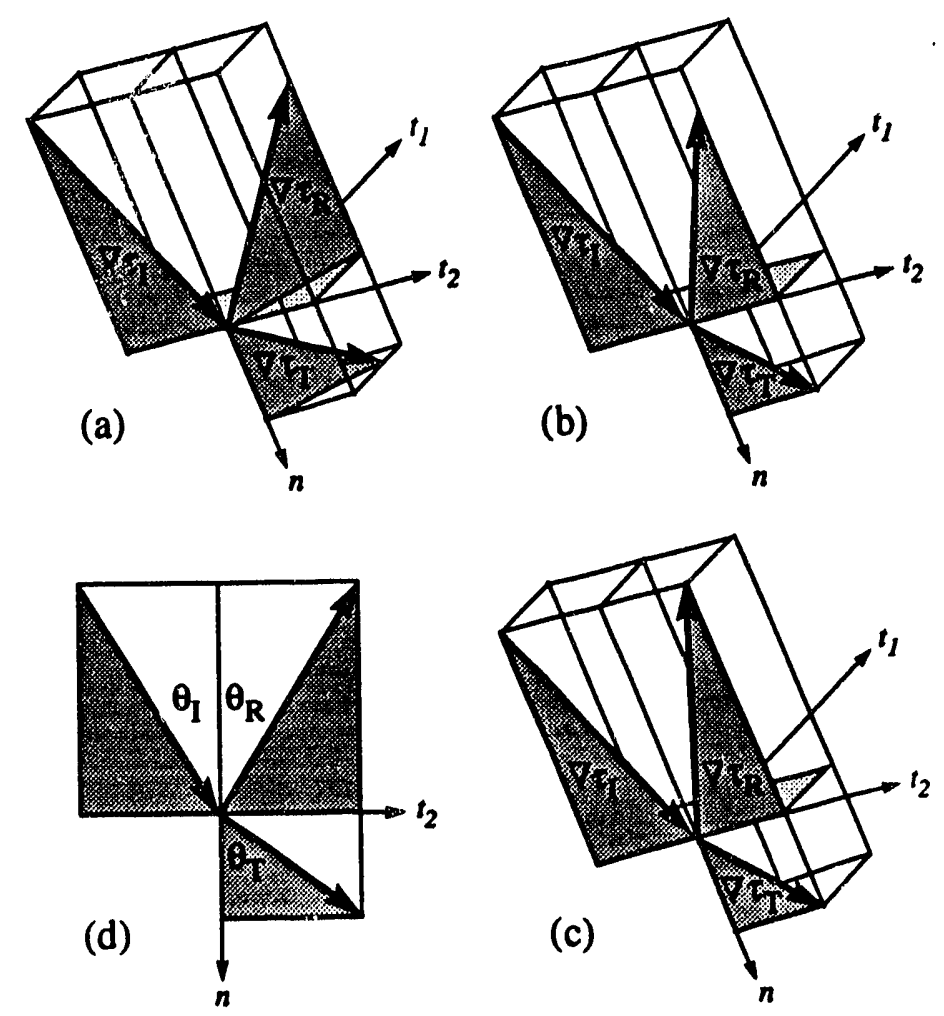

FIG. 7.3. Geometrical interpretation of traveltime gradients at a scattering interface in the $\left(t_{1}, t_{2}\right)$ plane. Equations (7.47) are satisfied for (a) a single tangent to the interface, in the $t_{2}$ direction, (b) the required arbitrary tangent direction, and (c) including the constraint from equation (7.51). The resulting angles that characterize the scattering geometry are shown in (d).

projections of the traveltime gradients into the scattering plane be equal for all definitions of the tangential gradient. Given the incident ray in Figure 7.3a, this constraint can be satisfied only if $\nabla \tau_{I}, \nabla \tau_{R}$, and $\nabla \tau_{T}$ lie in the plane defined by $\nabla \tau_{I}$ and $\hat{\boldsymbol{n}}$. Figure $7.3 \mathrm{~b}$ de- 
picts the proper geometrical interpretation of equations (7.47) for that particular incident ray.

Let us take

$$
\nabla_{t}=\nabla-\hat{n} \frac{\partial}{\partial n}
$$

as a particular choice of the tangent direction. To solve for $\partial \tau_{R} / \partial n$ in terms of $\partial \tau_{I} / \partial n$ we may then write from equations (7.47) that

$$
\nabla \tau_{I}-\hat{n} \frac{\partial \tau_{I}}{\partial n}=\nabla \tau_{R}-\hat{n} \frac{\partial \tau_{R}}{\partial n}
$$

First dot this equality by the vector $\nabla \tau_{I}$, then by $\nabla \tau_{R}$. Since $\tau_{I}$ and $\tau_{R}$ satisfy the same eikonal equation, the two resulting equations

$$
\begin{aligned}
& \left(\frac{\partial \tau_{I}}{\partial n}\right)^{2}=\sigma_{-}^{2}-\left[\nabla \tau_{I} \cdot \nabla \tau_{R}-\frac{\partial \tau_{I}}{\partial n} \frac{\partial \tau_{R}}{\partial n}\right] \\
& \left(\frac{\partial \tau_{R}}{\partial n}\right)^{2}=\sigma_{-}^{2}-\left[\nabla \tau_{I} \cdot \nabla \tau_{R}-\frac{\partial \tau_{I}}{\partial n} \frac{\partial \tau_{R}}{\partial n}\right],
\end{aligned}
$$

lead us to infer that

$$
\left(\frac{\partial \tau_{R}}{\partial n}\right)^{2}=\left(\frac{\partial \tau_{I}}{\partial n}\right)^{2}
$$

Without reference to either side of the interface,

$$
\sigma^{2} \stackrel{\text { def }}{=} \nabla \tau \cdot \nabla \tau
$$

defines the slowness of the medium.

We must choose

$$
\frac{\partial \tau_{R}}{\partial n}=-\frac{\partial \tau_{I}}{\partial n}
$$

to implement our physical notion that the incident and reflected rays should be oppositely directed. Figure $7.3 \mathrm{c}$ contains a drawing of rays that honors simultaneously equation (7.51) and equations (7.47).

Incident, reflection, and transmission ray angles may be introduced as in Figure 7.3d. According to equation (7.51) and equations (7.47) (or Figure 7.3c),

$$
\theta_{R}=\theta_{I}
$$

This relation of angles is the law of reflection. The law of refraction at an interface between two variable-density acoustic media is

$$
\sigma_{-} \sin \theta_{I}=\sigma_{+} \sin \theta_{T}
$$


We may also establish a relation between $\partial \tau_{k} / \partial n$ and the incident slowness $\sigma_{-}$. Employing equations (7.47) we first derive the relation

$$
\begin{aligned}
\left(\nabla_{t} \tau_{I}\right)^{2} & =\nabla_{t} \tau_{I} \cdot \nabla_{t} \tau_{R} \\
& =\nabla \tau_{I} \cdot \nabla \tau_{R}-\frac{\partial \tau_{I}}{\partial n} \frac{\partial \tau_{R}}{\partial n}
\end{aligned}
$$

then substitute into equations (7.49) to write

$$
\begin{aligned}
\frac{\partial \tau_{R}}{\partial n} & =-\frac{\partial \tau_{I}}{\partial n} \\
& =-\operatorname{sign}\left(\frac{\partial \tau_{I}}{\partial n}\right) \sqrt{\sigma_{-}^{2}-\left(\nabla_{t} \tau_{I}\right)^{2}}
\end{aligned}
$$

Equations (7.54) are the expressions we sought for the normal component of $\nabla \tau_{R}$ at an interface, needed for computation of scattering coefficients. In what follows I outline a derivation of the analogous result for $\nabla \tau_{T}$.

Remembering equation (7.48) as one choice for taking a tangential gradient I write

$$
\nabla \tau_{I}-\hat{n} \frac{\partial \tau_{I}}{\partial n}=\nabla \tau_{T}-\hat{n} \frac{\partial \tau_{T}}{\partial n}
$$

We dot these gradients, once by $\nabla \tau_{I}$ and again by $\nabla \tau_{T}$. Two equations result, analogous to equations (7.49), from which we decide that

$$
\begin{aligned}
\frac{\partial \tau_{T}}{\partial n} & =\operatorname{sign}\left(\frac{\partial \tau_{I}}{\partial n}\right) \sqrt{\sigma_{+}^{2}-\sigma_{-}^{2}+\left(\frac{\partial \tau_{I}}{\partial n}\right)^{2}} \\
& =\operatorname{sign}\left(\frac{\partial \tau_{I}}{\partial n}\right) \sqrt{\sigma_{+}^{2}-\left(\nabla_{t} \tau_{I}\right)^{2}}
\end{aligned}
$$

Equations (7.54) and (7.55) grant expression to reflection and transmission coeffcients entirely in terms of the incident field. Utilizing equations (7.51) and (7.55), and in accordance with equations (7.42) and (7.45),

$$
R_{0}=\frac{\frac{\partial \tau_{I}}{\partial n}-\operatorname{sign}\left(\frac{\partial \tau_{I}}{\partial n}\right) \sqrt{\sigma_{+}^{2}-\sigma_{-}^{2}+\left(\frac{\partial \tau_{I}}{\partial n}\right)^{2}}}{\frac{\partial \tau_{I}}{\partial n}+\operatorname{sign}\left(\frac{\partial \tau_{I}}{\partial n}\right) \sqrt{\sigma_{+}^{2}-\sigma_{-}^{2}+\left(\frac{\partial \tau_{I}}{\partial n}\right)^{2}}}, \quad\left(\Lambda_{\rho}>>\Lambda_{o}\right)
$$


and

$$
T_{0}=\frac{2 \frac{\partial \tau_{I}}{\partial n}}{\frac{\partial \tau_{I}}{\partial n}+\operatorname{sign}\left(\frac{\partial \tau_{I}}{\partial n}\right) \sqrt{\sigma_{+}^{2}-\sigma_{-}^{2}+\left(\frac{\partial \tau_{I}}{\partial n}\right)^{2}}}, \quad\left(\Lambda_{\rho}>>\Lambda_{o}\right)
$$

Suffice it to say that, for $\Lambda_{\rho} \approx \Lambda_{o}$, one obtains coefficients in terms of the incident ray by substituting equations (7.51) and (7.55) into equations (7.43), (7.44), and (7.46).

It is straightforward to derive from equation (7.39) that $R_{0}$ and $T_{0}$ are related through

$$
1+R_{0}=\left[\frac{\sqrt{\rho_{-}}}{\sqrt{\rho_{+}}}\right] T_{0}
$$

in agreement with the relation for plane waves. Again, however, equation (7.2) insists that this density ratio be set to unity, as was done in equation (7.57).

I shall conclude this derivation of the scattering of rays by examining equation (7.53) in the light of both available eikonal equations, equations (6.6) and (6.8). Direct substitution produces

$$
\begin{array}{cc}
\frac{\sin \theta_{I}}{c_{-}}=\frac{\sin \theta_{T}}{c_{+}}, & \left(\Lambda_{\rho}>>\Lambda_{o}\right), \\
\sin \theta_{I} \sqrt{\frac{1}{c_{-}^{2}}-\frac{1}{\omega^{2}}\left(\frac{\Delta \sqrt{\rho}}{\sqrt{\rho}}\right)_{-}}=\sin \theta_{T} \sqrt{\frac{1}{c_{+}^{2}}-\frac{1}{\omega^{2}}\left(\frac{\Delta \sqrt{\rho}}{\sqrt{\rho}}\right)_{+}}, \quad\left(\Lambda_{\rho} \approx \Lambda_{o}\right) .
\end{array}
$$

Equations (7.59) states Snell's Law with the same generality derived from plane-wave solutions, but qualified through the wavelength of the density function. When the dominant wavelength of density variation at an interface approaches that of the impinging acoustic field, ray bending depends on frequency and the density gradients - not simply on $c$. As might be hoped, the more general result reduces to its predecessor when frequency is very large or the dispersion coefficient is small. 


\section{Chapter 8}

\section{VARIABLE-DENSITY OPERATOR COMPARISONS}

We shall find it useful to collect our results, obtained in the preceeding material, on the analytical properties of the two variable-density differential operators. Let us compare the dispersion coefficient associated with each, review the conditions for stability and energy conservation, and summarize the density restrictions these operators impose at an interface between two continuous media. These density restrictions have practical ramifications for the accuracy of variable-density modeling. Last of all, I will also draw an analogy between these acoustic operators and elastic differential operators.

\subsection{Two acoustic operators}

The operator that arises from consistent management of the first principles of variable-density acoustics is, from DeSanto (1992),

$$
L^{(p)}=\rho \nabla \cdot\left[\frac{\nabla p}{\rho}\right]-\nabla \cdot\left[\frac{\nabla \rho}{\rho}\right] p-\frac{1}{c^{2}} \frac{\partial^{2} p}{\partial t^{2}}
$$

Most references (Claerbout, 1985; Wapenaar and Berkhout, 1989) take

$$
\tilde{L}^{(p)}=\rho \nabla \cdot\left[\frac{\nabla p}{\rho}\right]-\frac{1}{c^{2}} \frac{\partial^{2} p}{\partial t^{2}}
$$

as their variable-density operator. Both of these operators may be written in the KleinGordon form,

$$
L^{(q)}=\Delta q-\frac{b^{2}}{c^{2}} q-\frac{1}{c^{2}} \frac{\partial^{2} q}{\partial t^{2}}
$$

by substituting $q$ for $p, p=\sqrt{\rho} q$.

Based on its Klein-Gordon equivalent, the operator $L^{(p)}$ produces dispersive propagation according to the coefficient

$$
b^{2}=c^{2}\left[\frac{1}{2} \nabla \cdot\left(\frac{\nabla \rho}{\rho}\right)+\frac{\nabla \rho}{2 \rho} \cdot \frac{\nabla \rho}{2 \rho}\right] .
$$

For the operator $\tilde{L}^{(p)}, b^{2} \rightarrow \tilde{b}^{2}$, where

$$
\tilde{b}^{2}=c^{2}\left[\frac{1}{2} \nabla \cdot\left(\frac{\nabla \rho}{\rho}\right)-\frac{\nabla \rho}{2 \rho} \cdot \frac{\nabla \rho}{2 \rho}\right] .
$$


This dispersion coefficient, $\tilde{b}^{2}$, may be obtained directly by substituting $p$ for $q$ in the operator of equation (8.2).

The sign change in the brackets of equations (8.4) and (8.5) makes a difference in theory, but usually not in practice. The dispersion coefficient $b$ in equation (8.4) can be imaginary only if the divergence of fractional density is large and negative. To the contrary, the coefficient $\tilde{b}$ associated with equation (8.2) can be imaginary even if this divergence is positive. Significantly, analysis of a general initial-value problem-to determine conditions for well-posedness-predicts both of these operators can be unstable when dispersion is imaginary. However, this general analysis also predicts stability at large wavenumbers, no matter the sign of $b^{2}$. This is consistent with my solution to a specific, one-dimensional initial-value problem, where instability was limited to small wavenumbers $\left(k_{z}<\left|b_{o}\right| / c_{o}\right)$. Most exploration seismic data do not contain these wavenumbers.

Also at the root of this sign change, I showed from another point of view that the operator of equation (8.2) does not unconditionally conserve energy over time. Again, based on the one-dimensional example, I expect that growing, exponential solution components will be limited to small wavenumbers, not usually present in data.

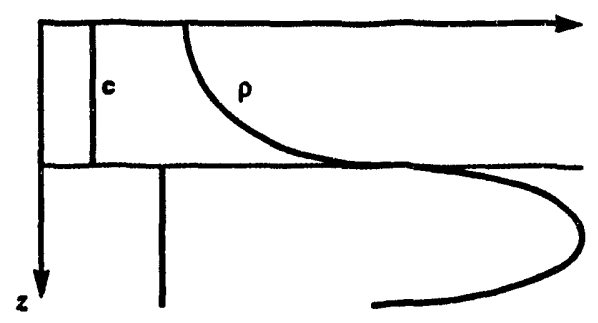

FIG. 8.1. Interpretation of the scattering coefficients associated with the operator of equation (8.1). This operator is valid for rapid density variation, with jumps at an interface in the speed, dispersion coefficient, and fractional density . Density must, however, be continuous in order to impart the proper scattered amplitudes.

One can examine further, through scattering coefficients, factors that impact the modeling accuracy of the operators of equations (8.1) and (8.2). That is, both of these operators place limits on the types of density variation that may be addressed-and still remain within their bounds of applicability. Figures 8.1 and 8.2 depict these bounds. Based on the work in Appendix A, I interpret these limits as the inevitable result of neglecting the force due to gravity. From neglecting this force, these op ritors are constrained by the physics of dynamic, rather than static, equilbrium for the initial state of the medium.

The operator of equation (8.1) is suited for rapid, continuous density variation in the medium, but precludes the possibility of discontinuous density profiles. To the contrary, one may only expect the operator of equation (8.2) to model accurately the wave response 


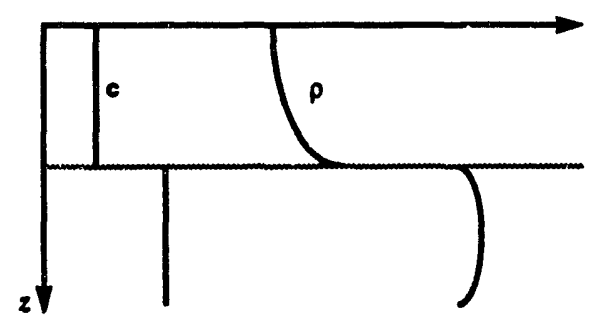

FIG. 8.2. Interpretation of the scattering coefficients associated with the operator of equation (8.2). This operator is valid for slow density variation, with jumps at an interface in the speed, dispersion coefficient, and fractional density . Density may also be discontinuous.

to slowly-varying density profiles (with negligible $\nabla \cdot(\nabla \rho / \rho)$ ). In return for this limitation one gains the legitimate freedom to consider discontinuities in density.

The error data plotted in Figure 3.2c underline the compromise I made in attempting to model acoustic waves in a medium having both large density gradients and density discontinuities (Figure 1.2). The Klein-Gordon operator $L^{(q)}$ (actually, its $\omega-k$ representation), employed to propagate the data in Figure 3.2b, is equivalent to $L^{(p)}$ and, hence, appropriate for $\Lambda_{\rho} / \Lambda_{o}<1$. However, lacking an alternative, I used the reflection coefficient (equation 7.33 ) associated with $\tilde{L}^{(p)}$ to scatter the propagating waves. Rapid density variation on either side of the scattering interface compromises the accuracy of that coefficient. One may discretize this model into constant-density subdivisions and use constant-density propagation and scattering (Figure 3.2a) to circumvent this dilemma.

\subsection{Elastic analogs}

Those for whom equation (8.1) is new may puzzle over the consequences of its additional-divergence of fractional density-term for elastic problems. In Appendix C I identify

$$
L^{(u)}=\left(\bar{c}_{i j k l} u_{k, l}^{(1)}+e_{i j}\left(\epsilon_{k l}\right)+w_{i j}\left(\omega_{k i}\right)\right)_{j}+\frac{\tau_{i j, j}^{(0)}}{\rho^{(0)}}\left(\rho^{(0)} u_{k}^{(1)}\right)_{, k}-\rho^{(0)} \frac{\partial^{2} u_{i}^{(1)}}{\partial t^{2}}
$$

as the elastic equivalent to $L^{(p)}$. Elastic variables $\bar{c}_{i j k i}, u_{i}^{(1)}$, and $\tau_{i j}^{(0)}$ denote, respectively, an effective elastic stiffness tensor, displacement perturbation, and the background stress distribution. The intrinsic stiffness tensor, $c_{i j k l}$, is altered by the background (initial) stresses in tie medium. The terms $e_{i j}$ and $w_{i j}$ depend, respectively, on the strain tensor, $\epsilon_{k l}$, and rotation tensor, $\omega_{k l}$, both defined in Appendix C. Due to its ad hoc nature I can find no exact elastic equivalent for equation (8.2). 
If the divergence of the initial stress vanishes in equation (8.6), then

$$
L^{(u)}=\left(\bar{c}_{i j k . l} u_{k, l}^{(1)}\right)_{, j}-\rho^{(0)} \frac{\partial^{2} u_{i}^{(1)}}{\partial t^{2}} .
$$

Equation (8.7) is employed commonly for small disturbances in an elastic solid (Aki and Richards, 1980). In Appendix C I conclude that this divergence should be neglected for those same elastic problems where one also neglects gravitational forces, forces due to Earth's rotation, forces due to tectonic stress, etc..

Equation (8.6) models, through the divergence of initial stress, an elastic, prestressed solid that is in dynamic, rather than static, equilibrium before waves are introduced. Importantly, the elastic equation of state does not impose this compromise on us-as does the acoustic equation. That is to say, we may neglect the gravitational force while modeling waves in variable-density elastic media, yet maintain a background in static equilibrium. I determined in Appendix $A$ that this is not possible for a variabledensity acoustic medium. Therefore, those who wish to do relevant modeling for prestressed, elastic media should include, for example, a static gravitational background term to pre-stress the medium, and take the background to be in static equilibrium (Dahlen, 1972).

In this paper I do not attempt to answer when and where those additional body forces may be neglected. Aki and Richards (1980) argue that they may be ignored for most seismic applications because they vary over wavelengths much longer than the seismic wavelength. On the other hand, John DeSanto (personal communication) describes problems in ocean acoustics where the effects of these forces, though small locally, accumulate over long propagation distances.

Nonetheless, the divergence of background stress in equation (8.6) takes on relevance in the acoustic case - even in the absence of gravitational forces, for example-and is required to proceed mathematically from elasticity to the operator of equation (8.1). That is to say, equation (8.7) leads directly to the constant-density acoustic wave equation on pressure.

Background stress, in an acoustic context, assumes its special relevance with respect to density due to an intimate relation between background pressure $\left(p^{(0)}\right)$ gradients and the density gradient. I derive, as a consequence of the acoustic equation of state,

$$
p_{, i}^{(0)}=c^{2} \rho_{, i}
$$

(equation (A-12)). We see that an acoustic medium cannot support a density gradient without a pressure gradient. We may conclude from this relation and

$$
p_{, i}^{(0)}=\tau_{i j, j}^{(0)}
$$

(see equation (C-17)) that the variable-density acoustic operator of equation (8.1) must hail from an elastic operator, equation (8.6), that involves the divergence of background stress. 


\section{Chapter 9}

\section{FUTURE WORK}

I mention two avenues along which one might expand the research in this thesis. The first is of a mathematical nature, the second, applied.

The Klein-Gordon operator provides a foundation for analyzing wave propagation in acoustic, inhomogeneous materials. I suggest that a similar, dispersive operator must also describe propagation in inhomogeneous elastic materials. Comparing the structure of the acoustic and elastic equations (equations (8.1) and (8.7)), I speculate that the elastic stiffness tensor will play a role in the elastic problem similar to that of density in the acoustic problem. The divergence operator acts on $\rho$ in the former equation, but on $\bar{c}_{i j k l}$ in the latter. I also suggest that one might make progress on the elastic problem by first considering asymptotic solutions. This was the approach used to identify the Klein-Gordon operator as the underlying description of the acoustic problem.

A second research direction involves utilization of the Klein-Gordon description for real materials. This description characterizes materials in terms of their speed and dispersion coefficient-rather than speed and density-emphasizing, in effect, the fundamental parameters that determine the wave response of inhomogeneous materials. Only idealized materials were considered in this work. A practicing geophysicist will probably find, for example, that metamorphic rocks are not constant-dispersion materials. One will have to determine, experimentally, if an accurate wave response can be obtained by approximating a region of the subsurface as a sequence of constant-dispersion units. If not, the next step would be an approach that allows the dispersion coefficient to vary continuously. 


\section{Chapter 10}

\section{CONCLUSIONS}

Theory, wave and ray, predicts that rapid density variation induces dispersive traveltime delays for signals propagating in an acoustic medium. This dispersion is of the Klein-Gordon variety, originating through a coefficient formed from the gradient and curvature of the density function. Dispersion is zero through a constant-density medium and media where $\sqrt{\rho}$ satisfies Laplace's equation.

These insights rest on a change of variable from pressure $p$ in the variable-density wave equation to $q$, where

$$
p=\sqrt{\rho} q .
$$

The quantity $q$ satisfies a Klein-Gordon differential equation, permitting analysis by Fourier transforms. That analysis identifies

$$
k^{2}=\frac{\omega^{2}}{c_{o}^{2}}-\left(\frac{\Delta \sqrt{\rho}}{\sqrt{\rho}}\right)_{0}
$$

as the dispersion relation appropriate for acoustic waves in variable-density media. Clearly, this relation governs variable-density traveltimes, as a simple amplitude term relates $p$ and $q$.

The term composed of derivatives on density endows the Klein-Gordon operator with its dispersive nature. One may interpret this coefficient as a mathematical description of the dispersion that builds up from multiple reflections of energy within discrete, constantdensity subdivisions of a continuously-varying medium. This dispersion is not considered by constant-density wave operators.

The traveltime aspect of this phenomenon is understood from ray theory in terms of an effective slowness. The righthand side of the eikonal equations

$$
\begin{aligned}
\nabla \tau \cdot \nabla \tau & =\frac{1}{c^{2}(x)}, & & \left(\Lambda_{\rho}>>\Lambda_{o}\right), \\
& =\frac{1}{c^{2}(x)}-\frac{1}{\omega^{2}}\left(\frac{\Delta \sqrt{\rho}}{\sqrt{\rho}}\right)_{o}, & & \left(\Lambda_{\rho} \approx \Lambda_{o}\right) .
\end{aligned}
$$

defines the slowness, squared, of an acoustic medium. Rapid density variation promotes density terms out of the transport equation (for amplitudes), and into the new, frequency-dependent eikonal on the lower line. Consequently, density variation on the same wavelength as the signal alters the effective slowness of the medium.

Since multiple eikonals necessarily represent the slowness of a variable-density medium, 
Snell's law-applied at an interface between two continuous media-requires multiple statements. That is,

$$
\begin{array}{ccc}
\frac{\sin \theta_{I}}{c_{-}} & =\frac{\sin \theta_{T}}{c_{+}}, & \left(\Lambda_{\rho}>>\Lambda_{o}\right), \\
\sin \theta_{I} \sqrt{\frac{1}{c_{-}^{2}}-\frac{1}{\omega^{2}}\left(\frac{\Delta \sqrt{\rho}}{\sqrt{\rho}}\right)_{-}}=\sin \theta_{T} \sqrt{\frac{1}{c_{+}^{2}}-\frac{1}{\omega^{2}}\left(\frac{\Delta \sqrt{\rho}}{\sqrt{\rho}}\right)_{+}}, \quad\left(\Lambda_{\rho} \approx \Lambda_{o}\right) .
\end{array}
$$

From the lower line, an interface between two media with different dispersion coefficients bends rays, even in the absence of a change in the characteristic speed $c$. That refraction vanishes for high frequencies.

Though not explicitly conveyed by the notation, these new ray-theoretical results for $\Lambda_{\rho} \approx \Lambda_{o}$ depend on the assumptions of slowly-varying speed and dispersion. I hasten to add that one must also make this supposition about $c$ to obtain the classical results $\left(\Lambda_{\rho} \gg \Lambda_{\circ}\right)$. Hence, I have extended the restrictions from slow variation in speed and density, so slow variation in speed and dispersion. These new results may thus be applied for those special geological environments where $c$ varies slowly, yet density varies rapidly. I give interbedded salt/anhydrite sections as one example.

We must ask, of course, what is the magnitude in real materials of these dispersive delays due to density variation? Importantly, I conclude from numerical experiments that the effective wave dispersion produced by discontinuities in density parameters (say, density or fractional density) far outweighs that due to continuous density variation. The Klein-Gordon dispersion relation describes the latter type of dispersion. Conditions for scattering at an interface impart the former type. Consequently, simple extensions of our common dispersion-relation based algorithms for modeling and imaging-from the constant-density relation to the Klein-Gordon relation-will not account properly for time delays imposed by rapid, discontinuous density variation. In order to account for these delays, an imaging algorithm must somehow incorporate a description of the scattering process, as well as the propagation process.

The comparison of two, different variable-density differential equations runs concurrently as a thread through this paper. These operators are

$$
L^{(p)}=\rho \nabla \cdot\left[\frac{\nabla p}{\rho}\right]-\nabla \cdot\left[\frac{\nabla \rho}{\rho}\right] p-\frac{1}{c^{2}} \frac{\partial^{2} p}{\partial t^{2}},
$$

and

$$
\tilde{L}^{(p)}=\rho \nabla \cdot\left[\frac{\nabla p}{\rho}\right]-\frac{1}{c^{2}} \frac{\partial^{2} p}{\partial t^{2}} .
$$

The differences in the properties of these operators stem from the divergence-of-fractionaldensity term, $\nabla \cdot(\nabla \rho / \rho) p$, neglected in the standard equation on the lower line. The 
Klein-Gordon operator,

$$
L^{(q)}=\Delta q-\frac{b^{2}}{c^{2}} q-\frac{1}{c^{2}} \frac{\partial^{2} q}{\partial t^{2}},
$$

subsumes both of these variable-density operators and provides an analytical foundation for comparisons.

Each of these operators are limited in certain respects, not all of practical importance. The usual initial-value problem-where one specifies initial data on a surface at $t=0$-is an ill-posed problem for these operators when the dispersion coefficient, $b$, is imaginary. (For example, for the variable-density operator that contains the divergence of fractional density, the dispersion coefficient is given by $b^{2}=c^{2} \Delta \sqrt{\rho} / \sqrt{\rho}$.) Importantly though, general analysis predicts stability at large wavenumbers. Specifically, the growing, exponential solutions that result (when $b$ is imaginary) are confined to wavenumber components $k_{z}<|b| / c$ in the analytic, one-dimensional solution that I computed. Most exploration seismic data do not contain these small wavenumbers.

Moreover, from an energy-analysis point of view, I conclude that the standard variable-density operator does not conserve energy when a density profile exhibits a nonzero divergence of fractional density. Again, based on the one-dimensional example, I expect that those exponential solution components that grow over time are limited to wavenumbers not present in real data.

The scattering coefficients associated with these operators underline, however, practical limitations. It seems that, thinking of a background medium in static equilibriumyet modeling with acoustic equations that imply dynamic equilibrium - we create density profiles for which both variable-density operators are flawed. This paradox arises because we neglect the force of gravity in the equations of motion.

To be specific, the non-standard operator is suited for rapid density variation in the medium, but its scattering coefficients preclude the possibility of discontinuous density at interfaces. To the conitrary, one may only expect the standard operator to model, accurately, the wave response to slowly-varying density profiles (with negligible divergence of fractional density). In return for this limitation one gains the legitimate freedom to consider discontinuities in density in an acoustic medium.

Some of the density models that I considered in this paper contain both rapid variation and jumps in density at interfaces. In these instances I compromised between these two operators. I propagated the waves, between discontinuities, with the Klein-Gordon equivalent of the non-standard equation. I scattered the waves at an interface based on the standard scattering coefficients. This approach works well for models where the wavelength $\Lambda_{\rho}$ of the density function exceeds the seismic wavelength, $\Lambda_{0}$. On the other hand, significant amplitude errors accumulate through models where $\Lambda_{\rho} / \Lambda_{o}<1$. The amplitudes recorded at the receiver position are in error by $50 \%$ for some of the models that I investigated. These errors are with respect to solutions calculated by constant-density propagation and scattering, using finely-discretized, constant-density subdivisions of the models. Requiring no density assumptions, I consider the latter solutions to be exact. 


\section{REFERENCES}

Abramowitz, M., and Stegun, I. A., 1965, Handbook of mathematical functions: Dover Publications Inc.

Aki, K., and Richards, P. G., 1980, Quantitative seismology: W.H. Freeman and C

Auld, B. A., 1990, Acoustic fields and waves in solids: Robert E. Krieger Publishing Co.

Bender, C. M., and Orszag, S. A., 1978, Advanced mathematical methods for scientists and engineers: McGraw-Hill Inc.

Ben-Menahem, A., and Singh, S. J., 1981, Seismic waves and sources: Springer-Verlag.

Bleistein, N., 1984, Mathematical methods for wave phenomena: Academic Press Inc.

Bracewell, R. N., 1986, The Fourier transform and its applications: McGraw-Hill Inc.

Brekhovskikh, L. M., 1960, Waves in layered media: Academic Press Inc.

Cerveny, V., Moltkov, I. A., and Psencik, I., 1977, Ray methods in seismology: Praha, Karlova Universita.

Claerbout, J. F., 1976, Fundamentals of geophysical data processing: McGraw-Hill Inc.

Claerbout, J. F., 1985, Imaging the earth's interior: Blackwell Scientific Publications.

Dahlen, F. A., 1972, Elastic dislocation theory for a self-gravitating elastic configuration with an initial static stress field: Geophys. J. Roy. Astr. Soc., 28, 357-383.

DeSanto, J. A., 1979, Derivation of the acoustic wave equation in the presence of gravitational and rotational effects: J. Acoust. Soc. Am., 66, 827-830.

DeSanto, J. A., 1992, Scalar wave theory-Green's functions and applications: Springer.

Dobrin, M. B., 1976, Introduction to geophysical prospecting: McGraw-Hill Inc.

Gazdag, J., 1978, Wave equation migration with the phase shift method: Geophysics, 43, 1342-1351.

Goupillaud, P. L, 1961, An approach to inverse filtering of near-surface layer effects from seismic records: Geophysics, 26, 754-760.

Gradshetyn, I. S. and Ryzhik, I. M., 1965, Table of integrals, series, and products: Academic Press Inc. 
Jackson, J. D., 1962, Classical Electrodynamics: John Wiley and Sons, Inc.

Jaunzemis, W., 1967, Continuum mechanics: MacMillan Company.

O'Doherty, R. F, 1971, Reflections on amplitudes: Geophys. Prosp., 19, 430-458.

Stakgold, I., 1979, Green's functions and boundary value problems: John Wiley and Sons, Inc.

Wapenaar, C. P. A., and Berkhout, A. J., 1989, Elastic wave field extrapolation: Elsevier.

Zemansky, M. W., 1968, Heat and thermodynamics: McGraw-Hill Kogakusha, Ltd. 


\section{Appendix A}

\section{VARIABLE-DENSITY WAVE EQUATION}

In this appendix I derive a linear wave equation for inhomogeneous acoustic media. The derived equation contains a fractional density term which is neglected in the standard wave equation. Though not derived in this appendix, this term is important to acoustic propagation times when the wavelength of the seismic wave is similar to the wavelength of density variation. Moreover, when the density function has curvature, the fractional density term is required for acoustic energy conservation.

Both variable-density equations (with and without the fractional density term) imply an acoustic background medium in dynamic-rather than static-equilibrium. This is important because we normally associate the standard equation with a static medium. However, because we neglect the force due to gravity in our equations of motion, we will find that the acoustic equation of state forces this dynamic interpretation upon us.

The derivation strategy shall stem from first principles. I write exact expressions for conservation of mass and linear momentum and choose an equation of state appropriate for fluids. Perturbation of these expressions gives a pair of linear differential field equations; a wave equation on pressure is derived from this system.

\section{A.1 Exact equations}

Consider a fixed volume $V$ of fluid surrounded by a surface $S$. An outward pointing unit normal vector $n$ gives the orientation of the surface at each point. Conservation of linear momentum is described for this fluid volume by

$$
\begin{aligned}
\frac{d}{d t} \int_{V} \rho v d V & =-\oint_{S} p n d S-\int_{V} f d V-\oint_{S} v \rho v \cdot \boldsymbol{n} d S \\
& =-\int_{V} \nabla p d V-\int_{V} f d V-\int_{V} \nabla \cdot(\boldsymbol{v} \rho v) d V
\end{aligned}
$$

where $p, v, \rho$, and $f$ respectively symbolize pressure, particle velocity, fluid density, and an acoustic source (body force density). The first two integrals on the right side of the equation calculate momentum gained per unit of time $t$. The last integral accounts for fluid flux out of the volume (momentum lost per unit time).

Since $V$ is arbitrary, equation (A-1) can be written in equivalent differential form as

$$
\frac{\partial(\rho v)}{\partial t}+\nabla \cdot(v \rho v)+\nabla p=-f .
$$


Expansion of the divergence gives

$$
\frac{\partial(\rho v)}{\partial t}+v \cdot \nabla \cdot(\rho v)+\rho v \cdot \nabla v+\nabla p=-f
$$

as recorded in Wapenaar and Berkhout (1989). Expansion of the time derivative shows that

$$
\boldsymbol{v}\left[\frac{\partial \rho}{\partial t}+\nabla \cdot(\rho v)\right]+\rho\left[\frac{\partial v}{\partial t}+\boldsymbol{v} \cdot \nabla v\right]+\nabla p=-f(x, t)
$$

The second bracketed term is referred to as a material or Lagrangian derivative on $\boldsymbol{v}$. Conservation of mass requires that the first bracketed term be zero.

In particular,

$$
\begin{aligned}
\frac{d}{d t} \int_{V} \rho d V & =-\oint_{S} \rho v \cdot n d S \\
& =-\int_{V} \nabla \cdot(\rho v) d V
\end{aligned}
$$

expresses mass conservation for the fluid volume as a whole. At a point, the differential equivalent

$$
\frac{\partial \rho}{\partial t}+\nabla \cdot(\rho v)=0
$$

holds. This treatment assumes no sources or sinks of mass within the volume.

Collecting results, conservation of linear momentum is written exactly (albeit, nonlinearly) as

$$
\rho\left[\frac{\partial v}{\partial t}+v \cdot \nabla v\right]+\nabla p=-f(x, t)
$$

Equation (A-5) gives three scalar relations in five unknown functions. Mass conversation (equation (A-4)) adis a fourth equation. An equation of state

$$
p=p(\rho)
$$

provides the necessary fifth, modeling compression/expansion of the fluid as an adiabatic process (Zemansky, 1968).

\section{A.2 Linear equations}

To proceed toward a linear wave equation, I linearize the system of five equations (A-4) - (A-6) by expanding all functions in perturbation series, as did DeSanto (1992). That is,

$$
\begin{aligned}
& \rho(x, t)=\rho^{(0)}+\epsilon \rho^{(1)}, \\
& p(x, t)=p^{(0)}+\epsilon p^{(1)}, \quad \text { and } \\
& v(x, t)=v^{(0)}+\epsilon v^{(1)},
\end{aligned}
$$


where $\epsilon$ is a small, dimensionless parameter. Background functions (those with a ${ }^{(0)}$ ) are interpreted as descriptions of the medium before waves are introduced. Perturbations from the background state are due to waves.

Substitution of equations (A-7) into equation (A-4) gives linear, ordered expressions for mass conservation. That is to say, to zeroth order in $\epsilon$

$$
\frac{\partial \rho^{(0)}}{\partial t}+\nabla \cdot\left(\rho^{(0)} v^{(0)}\right)=0
$$

and, to $O\left(\epsilon^{1}\right)$,

$$
\frac{\partial \rho^{(1)}}{\partial t}+\nabla \cdot\left(\rho^{(0)} v^{(1)}\right)+\nabla \cdot\left(\rho^{(1)} v^{(0)}\right)=0
$$

Substitution of equations (A-7) into the expression for momentum conservation (equation (A-5)) produces

$$
\rho^{(0)} \frac{\partial v^{(0)}}{\partial t}+\nabla p^{(0)}=\mathbf{0}
$$

to $O(1)\left(\right.$ where $\left.f^{(0)} \equiv 0\right)$ and

$$
\rho^{(0)} \frac{\partial v^{(1)}}{\partial t}+\rho^{(1)} \frac{\partial v^{(0)}}{\partial t}+\nabla p^{(1)}=-f^{(1)}
$$

to $O\left(\epsilon^{1}\right)$.

Quadratic velocity terms were neglected to obtain linear expressions. However, $v^{(0)}$ was not taken to be zero, as is typically the case (Wapenaar and Berkhout, 1989). Analysis of the equation of state $(A-6)$ reveals $v^{(0)} \equiv 0$ to be inconsistent with a variabledensity acoustic medium, as I now show.

We shall follow the analysis of DeSanto (1992). Taking $p^{(0)}=p^{(0)}\left(\rho^{(0)}\right)$ the chain rule gives

$$
\nabla p^{(0)}=c^{2} \nabla \rho^{(0)}
$$

with

$$
c^{2} \stackrel{\text { def }}{=} \frac{\partial p^{(0)}}{\partial \rho^{(0)}} .
$$

The assumption $v^{(0)} \equiv 0$ requires $\nabla p^{(0)} \equiv 0$ from equation $(\mathrm{A}-10)$, and equation $(\mathrm{A}-12)$ in turn requires $\nabla \rho^{(0)} \equiv 0$. That is to say, this inhomogeneous fluid medium is, to zeroth order, in hydrodynamic equilibrium. Neglecting the force due to gravity, hydrostatic equilibrium $\left(v^{(0)} \equiv 0\right)$ is not permissible for variable-density acoustic media.

Equation (A-12) will be used to trade background pressure gradients for density gradients, density being the quantity measured routinely in exploration geophysics. Again, I follow DeSanto (1992) to derive another substitution. Expanding pressure about the background state $p^{(0)}\left(\rho^{(0)}\right)$ and using equations $(\mathrm{A}-7)$,

$$
p^{(0)}+\epsilon p^{(1)}=p^{(0)}+\frac{\partial p^{(0)}}{\partial \rho^{(0)}}\left(\rho-\rho^{(0)}\right)
$$




$$
=p^{(0)}+\epsilon \frac{\partial p^{(0)}}{\partial \rho^{(0)}} \rho^{(1)}
$$

Therefore,

$$
p^{(1)}=c^{2} \rho^{(1)}
$$

to $O\left(\epsilon^{1}\right)$. This result gives a substitution for density perturbations in terms of pressure perturbations.

One additional physical simplification is assumed customarily for exploration geophysics problems. One normally assumes $\partial \rho^{(0)} / \partial t \equiv 0$ (or, $\rho^{(0)}=\rho^{(0)}(x)$ at most). This implies that, to zeroth order, the fluid is incompressible and can not flux out of the volume. Proof of these physical consequences depends on liberal use of the divergence theorem. From equation $(\mathrm{A}-8), \nabla \cdot\left(\rho^{(0)} v^{(0)}\right) \equiv 0$ implies $\oint_{S}\left(\rho^{(0)} v^{(0)} \cdot n\right) d S \equiv 0$. Since $\boldsymbol{V}$ is arbitrary, these identities can be satisfied only if $\boldsymbol{v}^{(0)} \cdot \boldsymbol{n}=\mathbf{0}$ everywhere on $S$. This logic applied to equation (A-9) yields

$$
\frac{\partial \rho^{(1)}}{\partial t}+\nabla \cdot\left(\rho^{(0)} v^{(1)}\right)=0
$$

Also, if $p^{(0)}=p^{(0)}\left[\rho^{(0)}(x)\right]$ then $c=c(x)$ at most, from equation (A-13).

Equations (A-10) through (A-15) constitute the system of linearized expressions needed to write a linear wave equation on a single field (perturbation) quantity. Customarily, one chooses to write the equation for pressure. Density perturbation terms are replaced with pressure perturbations in equations $(A-15)$ and $(A-11)$ using equation (A-14). All background functions are then expressed in terms of background density through equations $(A-10)$ and $(A-12)$. These substitutions give

$$
\frac{1}{c^{2}} \frac{\partial p^{(1)}}{\partial t}+\nabla \cdot\left(\rho^{(0)} v^{(1)}\right)=0
$$

and

$$
\rho^{(0)} \frac{\partial v^{(1)}}{\partial t}-\frac{\nabla \rho^{(0)}}{\rho^{(0)}} p^{(1)}+\nabla p^{(1)}=-f^{(1)}(x, t) .
$$

The above pair of differential equations are the field equations for linear waves in inhomogeneous acoustic media. Those equations are respective statements of mass conservation in combination with the acoustic equation of state, and conservation of momentum. The wave equation

$$
\Delta p^{(1)}-\nabla \cdot\left[\frac{\nabla \rho^{(0)}}{\rho^{(0)}} p^{(1)}\right]-\frac{1}{c^{2}(x)} \frac{\partial^{2} p^{(1)}}{\partial t^{2}}=-\nabla \cdot f^{(1)}(x, t)
$$

results from the divergence of equation (A-17) minus the time derivative of equation (A-16). This result is recognized to be the wave equation for a constant-density medium but, with an additional term, the term with brackets. 
Another form of the variable-density wave equation is more common. Expand the bracketed term in equation $(\mathrm{A}-18)$ and factor out $\rho^{(0)}$ from terms containing differentiated pressure. These manipulations produce the form

$$
\rho^{(0)}(x) \nabla \cdot\left[\frac{\nabla p^{(1)}}{\rho^{(0)}}\right]-\nabla \cdot\left[\frac{\nabla \rho^{(0)}}{\rho^{(0)}}\right] p^{(1)}-\frac{1}{c^{2}(x)} \frac{\partial^{2} p^{(1)}}{\partial t^{2}}=-\nabla \cdot f^{(1)}(x, t),
$$

where

$$
\left[\frac{\nabla}{\rho^{(0)}}\right]
$$

is a fractional density. Though this term is often neglected (Claerbout, 1985; Wapenaar and Berkhout, 1989), we showed that the differentiated equation of state (A-12) and equation (A-10) require, strictly speaking, that it be included.

Equation (A-19) is the final wave equation form sought in this development. Traditionally, background and perturbation superscripts are dropped.

\section{A.3 Velocity potential}

We shall find a velocity potential $\psi^{(1)}$ particularly useful for forming energy expressions. I will first derive its relation with $p^{(1)}$, then derive the corresponding differential operator.

We begin with the basic definition,

$$
-\nabla \psi^{(1)} \stackrel{\text { def }}{=} v^{(1)}
$$

Next, substitute this definition into equation (A-11) and, for convenience, take the source term to be zero. Additional substitutions from equations (A-10), (A-12), and (A-14) result in

$$
\begin{aligned}
\nabla\left[\frac{\partial \psi^{(1)}}{\partial t}\right] & =\frac{\nabla p^{(1)}}{\rho^{(0)}}-\frac{\nabla \rho^{(0)}}{\left(\rho^{(0)}\right)^{2}} p^{(1)} \\
& =\nabla\left[\frac{p^{(1)}}{\rho^{(0)}}\right]
\end{aligned}
$$

We can conclude that

$$
p^{(1)}=\rho^{(0)} \frac{\partial \psi^{(1)}}{\partial t}
$$

gives the relation between perturbations in pressure and velocity potential.

To determine the appropriate operator for $\psi^{(1)}$ we may substitute equation (A-22) into equation (A-19). This substitution produces

$$
\rho^{(0)} \frac{\partial}{\partial t}\left\{\nabla \cdot\left[\frac{\nabla\left(\rho^{(0)} \psi^{(1)}\right)}{\rho^{(0)}}\right]-\nabla \cdot\left[\frac{\nabla \rho^{(0)}}{\rho^{(0)}}\right] \psi^{(1)}-\frac{1}{c^{2}} \frac{\partial^{2} \psi^{(1)}}{\partial t^{2}}\right\}=-\nabla \cdot f^{(1)}
$$


It is enlightening to expand the leftmost term in braces as

$$
\nabla \cdot\left[\frac{\nabla\left(\rho^{(0)} \psi^{(1)}\right)}{\rho^{(0)}}\right]=\frac{1}{\rho^{(0)}} \nabla \cdot\left[\rho^{(0)} \nabla \psi^{(1)}\right]+\nabla \cdot\left[\frac{\nabla \rho^{(0)}}{\rho^{(0)}}\right] \psi^{(1)} .
$$

We see that the fractional density term in equation (A-24) cancels an identical term in equation (A-23), and it's a straightforward matter to reason that

$$
\frac{1}{\rho^{(0)}} \nabla \cdot\left[\rho^{(0)} \nabla \psi^{(1)}\right]-\frac{1}{c^{2}} \frac{\partial^{2} \psi^{(1)}}{\partial t^{2}}=-\frac{1}{\rho^{(0)}} \nabla \cdot f_{(t)}^{(1)} .
$$

The symbol $f_{(t)}^{(1)}$ denotes time integration,

$$
f_{(t)}^{(1)} \stackrel{\text { def }}{=} \int^{t} d t^{\prime} f^{(1)}
$$

Equation (A-25) defines the appropriate differential operator on velocity potential-when $\nabla \cdot\left(\nabla \rho^{(0)} / \rho^{(0)}\right) p^{(1)}$ is included in the variable-density operator.

When one excludes that fractional density term (from the variable-density operator of equation (A-19)),

$$
\frac{1}{\rho^{(0)}} \nabla \cdot\left[\rho^{(0)} \nabla \psi^{(1)}\right]+\nabla \cdot\left[\frac{\nabla \rho^{(0)}}{\rho^{(0)}}\right] \psi^{(1)}-\frac{1}{c^{2}} \frac{\partial^{2} \psi^{(1)}}{\partial t^{2}}=-\frac{1}{\rho^{(0)}} \nabla \cdot f_{(t)}^{(1)} .
$$

This result follows easily by going back to equation (A-23) and dropping the divergence of fractional density. Proceeding again from that point, fractional densities no longer cancel out of the velocity potential operator. 


\section{Appendix B}

\section{AN ANALYTIC KLEIN-GORDON SOLUTION}

As derived in the text,

$$
q(z, t)=\frac{1}{2 \pi} \frac{1}{\sqrt{\rho\left(z^{\prime}\right)}} H(t) \sum_{ \pm} \int_{-\infty}^{\infty} d k_{z} e^{i\left[k_{z}\left(z-z^{\prime}\right) \mp \sqrt{b_{o}^{2}+c_{o}^{2} k_{z}^{2}} t\right]}
$$

is the solution to a Klein-Gordon initial-value problem, described by

$$
\begin{gathered}
\frac{\partial^{2} q}{\partial z^{2}}-\frac{b^{2}}{c^{2}} q-\frac{1}{c^{2}} \frac{\partial^{2} q}{\partial t^{2}}=0 \\
q(z, 0)=\frac{1}{\sqrt{\rho}} \delta\left(z-z^{\prime}\right), \quad \frac{\partial}{\partial t} q(z, 0)=0
\end{gathered}
$$

In this appendix we will evaluate the integrals in equation (B-1).

Preferring to work with convergent integrals, I set

$$
q(z, t)=\frac{1}{2 \pi} \frac{1}{\sqrt{\rho\left(z^{\prime}\right)}} H(t) \sum_{ \pm} \pm \frac{1}{i} \frac{\partial \tilde{q}}{\partial t}
$$

where

$$
\tilde{q}(z, t) \stackrel{\text { def }}{=} \int_{-\infty}^{\infty} d k_{z} \frac{e^{i\left[k_{z}\left(z-z^{\prime}\right) \mp \sqrt{b_{o}^{2}+c_{o}^{2} k_{z}^{2}} t\right]}}{\sqrt{b_{0}^{2}+c_{o}^{2} k_{z}^{2}}} .
$$

The strategy for evaluating the integrals of equation (B-4) is first, suppress the square roots via a change in the integration variable, then, combine terms in the exponest.

Let us take

$$
c_{o} k_{z}=b_{c} \sinh \Omega
$$

Consequently,

$$
\hat{q}(z, t)=\frac{1}{c_{o}} \int_{-\infty}^{\infty} d \Omega e^{i b_{0}\left[1 / c_{o}\left(z-z^{\prime}\right) \sinh \Omega \mp t \cosh \Omega\right]} .
$$

Now terms in the exponent may be combined as

$$
\frac{z-z^{\prime}}{c_{o}} \sinh \Omega \mp t \cosh \Omega=\mp \frac{\zeta}{c_{o}} \cosh (\Omega \mp \beta),
$$

where

$$
\zeta \stackrel{\text { def }}{=} \sqrt{c_{o}^{2} t^{2}-\left(z-z^{\prime}\right)^{2}}
$$


and

$$
\sinh \beta \stackrel{\text { def }}{=} \frac{z-z^{\prime}}{\zeta}, \quad \cosh \beta \stackrel{\text { def }}{=} \frac{c_{o} t}{\zeta}, \quad \cosh ^{2} \beta-\sinh ^{2} \beta=1 .
$$

These substitutions in equation (B-5) give

$$
\tilde{q}(z, t)=\frac{1}{c_{o}} \int_{-\infty}^{\infty} d \Omega e^{\mp i\left(b_{o} / c_{o}\right) \zeta \cosh (\Omega \mp \beta)}
$$

The phase shift $\beta$ does not contribute to the integral. One can see this by setting $\Omega^{\prime}=\Omega \mp \beta$, finding that $d \Omega^{\prime}=d \Omega$, with no change in the limits of integration. We may therefore drop $\beta$.

The solutions to equation (B-7) are defined to be Hankel functions (Gradshteyn and Ryzhik, 1965), $H_{0}^{(1)}$ and $H_{0}^{(2)}$, for $\zeta>0$. Consequently,

$$
\tilde{q}(z, t)= \begin{cases}-\frac{\pi i}{c_{o}} H\left(\zeta^{2}\right) H_{0}^{(2)}\left(\frac{b_{o}}{c_{o}} \zeta\right) & , \text { for } e^{-i \ldots} \\ +\frac{\pi i}{c_{o}} H\left(\zeta^{2}\right) H_{0}^{(1)}\left(\frac{b_{o}}{c_{o}} \zeta\right) & , \text { for } e^{+i \ldots} .\end{cases}
$$

I have appended a Heaviside distribution, $H\left(\zeta^{2}\right)$, to these solutions. This distribution characterizes the fact that $\tilde{q}$ is given by Hankel functions only inside the domain of influence of the point $\left(z^{\prime}, t=0\right)$, and is zero outside. Differentiated over time,

$$
\frac{\partial \tilde{q}}{\partial t}= \begin{cases}-\pi i \frac{c_{o} t}{\zeta}\left[2 \delta\left(\zeta^{2}\right) H_{0}^{(2)}\left(\frac{b_{o}}{c_{o}} \zeta\right)-\frac{1}{2} \frac{b_{o}}{c_{o}} H\left(\zeta^{2}\right) H_{1}^{(2)}\left(\frac{b_{o}}{c_{o}} \zeta\right)\right] & , \text { for } e^{-i \ldots} \\ +\pi i \frac{c_{o} t}{\zeta}\left[2 \delta\left(\zeta^{2}\right) H_{0}^{(1)}\left(\frac{b_{o}}{c_{o}} \zeta\right)-\frac{1}{2} \frac{b_{o}}{c_{o}} H\left(\zeta^{2}\right) H_{1}^{(1)}\left(\frac{b_{o}}{c_{o}} \zeta\right)\right] & , \text { for } e^{+i \ldots}\end{cases}
$$

The symbol $\delta\left(\zeta^{2}\right)$ is the Dirac delta function.

With equations (B-3) and (B-9) taken together,

$$
q(z, t)=\frac{1}{\sqrt{\rho\left(z^{\prime}\right)}} H(t) \frac{c_{o} t}{\zeta}\left[-\delta\left(\zeta^{2}\right) H_{0}^{(2)}+\frac{1}{4} \frac{b_{o}}{c_{o}} H\left(\zeta^{2}\right) H_{1}^{(2)}-\delta\left(\zeta^{2}\right) H_{0}^{(1)}+\frac{1}{4} \frac{b_{o}}{c_{o}} H\left(\zeta^{2}\right) H_{1}^{(1)}\right]
$$

That is,

$$
\begin{gathered}
q(z, t)=\frac{1}{\sqrt{\rho\left(z^{\prime}\right)} H(t) \frac{c_{o} t}{\zeta}\left[-2 \delta\left(\zeta^{2}\right)+\frac{1}{2} \frac{b_{o}}{c_{o}} H\left(\zeta^{2}\right) J_{1}\left(\frac{b_{o}}{c_{o}} \zeta\right)\right],} \\
\zeta \stackrel{\text { def }}{=} \sqrt{c_{o}^{2} t^{2}-\left(z-z^{\prime}\right)^{2}}, \quad \zeta>0, \quad b_{o}^{2}>0 .
\end{gathered}
$$

$J_{1}$ is a Bessel function, and is proportional to the sum of $H_{1}^{(1)}$ and $H_{1}^{(2)}$. I have evaluated $J_{0}$ at the support of the delta function. 


\section{Appendix C}

\section{ACOUSTIC-ELASTIC ANALOGS}

In the development that follows I identify

$$
\left(\bar{c}_{i j k l} u_{k, l}^{(1)}+e_{i j}\left(\epsilon_{k l}\right)+w_{i j}\left(\omega_{k l}\right)\right)_{, j}+\frac{\tau_{i j, j}^{(0)}}{\rho^{(0)}}\left(\rho^{(0)} u_{k}^{(1)}\right)_{, k}-\rho^{(0)} \frac{\partial^{2} u_{i}^{(1)}}{\partial t^{2}}=f_{i}^{(1)}
$$

as the elastic analogy to the acoustic equation - equation (A-19)-derived in Appendix A. Elastic variables $\bar{c}_{i j k l}, u_{i}^{(1)}$, and $\tau_{i j}^{(0)}$ denote ${ }_{\gamma}$ respectively, an effective elastic stiffness tensor, displacement perturbation, and the background stress distribution. The intrinsic atiffness tensor, $c_{i j k l}$, is altered by the background (initial) stresses in the medium. The terms $e_{i j}$ and $w_{i j}$ depend, respectively, on the strain tensor, $\epsilon_{k l}$, and rotation tensor, $\omega_{k l}$, where

$$
\epsilon_{k l} \stackrel{\text { def }}{=} \frac{1}{2}\left(u_{k, l}^{(1)}+u_{l, k}^{(1)}\right)
$$

and

$$
\omega_{k l} \stackrel{\text { def }}{=} \frac{1}{2}\left(u_{k, l}^{(1)}-u_{l, k}^{(1)}\right)
$$

Explicitly,

$$
e_{i j}=\epsilon_{i k} \tau_{k j}^{(0)}+\tau_{i k}^{(0)} \epsilon_{k j}
$$

and

$$
w_{i j}=\omega_{i k} \tau_{k j}^{(0)}-\tau_{i k}^{(0)} \omega_{k j} .
$$

One may, as well, write equation (A-19) in the tensor notation, used here. In this notation,

$$
\rho^{(0)}\left(\frac{p_{, j}^{(1)}}{\rho^{(0)}}\right)_{, j}-\left(\frac{\rho_{, j}^{(0)}}{\rho^{(0)}}\right)_{j} p^{(1)}-\frac{1}{c^{2}} \frac{\partial^{2} p^{(1)}}{\partial t^{2}}=-f_{j, j}^{(1)} .
$$

If the divergence of the initial stress vanishes in equation (C-1), then

$$
\left(\bar{c}_{i j k l} u_{k, l}^{(1)}\right)_{. j}-\rho^{(0)} \frac{\partial^{2} u_{i}^{(1)}}{\partial t^{2}}=f_{i}^{(1)},
$$

from equations (C-4) and (C-5). Equation (C-7) is employed commonly for small disturbances in an elastic solid (Aki and Richards, 1980).

In this appendix I conclude that this divergence should be neglected for those elastic problems where one also neglects gravitational forces, forces due to Earth's rotation, forces due to tectonic stress, etc.. Aki and Richards (1980) argue that these forces may be ignored for most seismic applications because they vary over wavelengths much longer 
than the seismic wavelength.

Equation (C-1) models, through the divergence of initial stress, an elastic, prestressed solid that is in dynamic, rather than static, equilibrium before waves are introduced. Importantly, the elastic equation of state does not impose this compromise on us-as does the acoustic equation. That is to say, we may neglect the gravitational force while modeling waves in inhomogeneous elastic media, yet maintain a background in static equilibrium. I determined in Appendix A that this is not possible for a variabledensity acoustic medium. Therefore, those who wish to do modeling for pre-stressed, elastic media should include, for example, a static gravitational background term to pre-stress the medium (Dahlen, 1972).

Nevertheless, the divergence of background stress takes on relevance in the acoustic case - even in the absence of, e.g., gravitational forces-and is required to proceed mathematically from elastics to equation (C-6). Equation (C-7) leads directly to the constant-density acoustic wave equation on pressure.

In the acoustic setting, background stress assumes its special importance with respect to density due to an intimate relation between background pressure gradients and the density gradient. I showed in Appendix A that, by neglecting the force due to gravity, we are required to accept the notion of a dynamic background in order to have a variable-density medium. On the other hand, I show in this appendix that this divergence of stress does not likewise constrain whether the acoustic speed $c$ may vary over space.

\section{C.1 Exact elastic equations}

Let us derive equation (C-1). The integral equations

$$
\begin{aligned}
\frac{d}{d t} \int_{V} \rho v_{i} d V & =\oint_{S} T_{i} d S-\int_{V} f_{i} d V-\oint_{S} v_{i} \rho v_{j} n_{j} d S \\
& =\int_{V} \tau_{i j, j} d V-\int_{V} f_{i} d V-\int_{V}\left(v_{i} \rho v_{j}\right)_{j} d V
\end{aligned}
$$

express conservation of linear momentum in an elastic solid. $T_{i}$ symbolizes the traction vector, and is related to the stress tensor through

$$
T_{i}=\tau_{i j} n_{j} .
$$

In the equivalent differential form,

$$
\rho\left[\frac{\partial v_{i}}{\partial t}+\imath_{j} v_{i, j}\right]-\tau_{i j, j}=-f_{i} .
$$

Conservation of mass (equation (A-4)) has been posited in proceeding from equation (C-8) to equation (C-9). 


\section{C.2 Linear elastic equations}

One may linearize equation (C-9) by employing the perturbation expansions of equations (A-7) and

$$
\tau_{i j}(x, t)=\tau_{i j}^{(0)}+\epsilon \tau_{i j}^{(1)} .
$$

These substitutions in equation (C-9) produce

$$
\rho^{(0)} \frac{\partial v_{i}^{(0)}}{\partial t}-\tau_{i j, j}^{(0)}=-f_{i}^{(0)},
$$

to $O(1)$, and

$$
\rho^{(0)} \frac{\partial v_{i}^{(1)}}{\partial t}+\rho^{(1)} \frac{\partial v_{i}^{(0)}}{\partial t}-\tau_{i j, j}^{(1)}=-f_{i}^{(1)}
$$

to $O\left(\epsilon^{1}\right)$. All terms that are quadratic in velocity were neglected.

Dahlen (1972) assumes that the elastic medium is in a state of static equilibrium $\left(\partial v_{i}^{(0)} / \partial t \equiv 0\right)$ prior to passage of a wave. He sets $\tau_{i j, j}^{(0)}$, in equation (C-10), equal to body forces due to gravitation and the earth's rotation. These choices for pre-stressing a solid make a lot of physical sense. DeSanto (1979) makes analogous choices for a fluid.

Nevertheless, I shall set $f_{i}^{(0)}$ to zero and require nonzero $\partial v_{i}^{(0)} / \partial t$, in order to honor the acoustic conditions represented by equation (C-6), which does not include gravitational effects. Once again neglecting gravity in equation (C-10), we are forced to accept an initial state of dynamic equilibrium in order to maintain an analogy with equation (C-6). That is, equation (C-1) describes motion in an elastic medium that was pre-stressed due to forces of dubious origin-forces in dynamic equilibrium. I will return to an acoustic-elastic comparison after reducing the elastic wave equation $(\mathrm{C}-1)$ to its acoustic counterpart.

To complete the derivation of equation (C-1) we use equations (A-15) and (C-10) to substitute for $\rho^{(1)} \partial v_{i}^{(0)} / \partial t$ in equation (C-11). Hence we write

$$
\rho^{(0)} \frac{\partial^{2} u_{i}^{(1)}}{\partial t^{2}}-\frac{\tau_{i j, j}^{(0)}}{\rho^{(0)}}\left(\rho^{(0)} u_{k}^{(1)}\right)_{, k}-\tau_{i j, j}^{(1)}=-f_{i}^{(1)}
$$

for the balance of perturbation forces. The displacement perturbation $u_{i}^{(1)}$ is defined through

Hooke's law,

$$
v_{i}^{(1)}=\frac{\partial u_{i}^{(1)}}{\partial t} .
$$

$$
\tau_{i j}^{(1)}=c_{i j k l} u_{k, l}^{(1)}+e_{i j}+w_{i j},
$$

for incremental elastic strain in a pre-stressed medium, transforms equation (C-12) into equation (C-1). Aki and Richards (1980) allude to equation (C-13). I followed Jaunzemis (1967) to derive it. 


\section{C.3 Acoustic equivalents}

Let us reduce equation (C-1) to its acoustic equivalent. First put (Ben-Menahem and Singh,1981)

$$
\bar{c}_{i j k l}=\bar{\lambda} \delta_{i j} \delta_{k l},
$$

where $\bar{\lambda}$ is Lame's constant. Furthermore; the rotation $\omega_{k l}$ and, hence, $w$, vanish in fluids. We may now calculate

$$
\begin{aligned}
\left(\bar{c}_{i j k l} u_{k, l}^{(1)}+e_{i j}\right)_{, j} & =\left(\bar{\Lambda} u_{k, k}^{(1)}\right)_{, i} \\
& =-p_{, i}^{(1)} .
\end{aligned}
$$

The first equality of equations (C-15) follows from the work of Jaunzemis (1967), and Ben-Menahem and Singh (1981) establish the second. Symbol $\bar{\Lambda}$ denot os an effective Lame's constant, which depends both on $\bar{\lambda}$ and the initial pressure $p^{(0)}$.

Next I consider the acoustic specialization of the background-stress term of equation (C-1). One finds that

$$
\begin{aligned}
\frac{\tau_{i j, j}^{(0)}}{\rho^{(0)}}\left(\rho^{(0)} u_{k}^{(1)}\right)_{, k} & =\frac{\rho^{(1)}}{\rho^{(0)}} p_{, i}^{(0)} \\
& =\frac{1}{\rho^{(0)} c^{2}} p_{, i}^{(0)} p^{(1)} \\
& =\frac{\rho_{i}^{(0)}}{\rho^{(0)}} p^{(1)}
\end{aligned}
$$

I used, in this order,

$$
\tau_{i j}^{(0)}=-p^{(0)} \delta_{i j},
$$

and equations (A-15), (A-14), and (A-12) to determine these equalities.

Collecting these results, we now introduce equations (C-15) and (C-16) into equation (C-1). I obtain

$$
-p_{. i}^{(1)}+\frac{\rho_{. i}^{(0)}}{\rho^{(0)}} p^{(1)}-\rho^{(0)} \frac{\partial^{2} u_{i}^{(1)}}{\partial t^{2}}=f_{i}^{(1)} .
$$

If we now take the divergence of each term and employ equations (A-15) and (A-14) to rewrite the divergence of the inertial term, then

$$
p_{, i i}^{(1)}-\left(\frac{\rho_{, i}^{(0)}}{\rho^{(0)}} p^{(1)}\right)_{, i}-\frac{1}{c^{2}} \frac{\partial^{2} p^{(1)}}{\partial t^{2}}=-f_{i, i}^{(1)} .
$$

Equation (C-19) is the equivalent, in tensor notation, of equation (A-18). One may recombine the terms of equation (C-19), as was done in Appendix A, to see its strict equivalence with equation $(C-6)$. We have therefore shorn that equation $(C-1)$ represents the elastic analog of equation (C-6). 
What, then, is the analog of the standard elastic wave equation, equation (C-7)? Consistent with $\tau_{i j, j}^{(0)}=0$, equations (C-15) simplify to

$$
\begin{aligned}
\left(\bar{c}_{i j k l} u_{k, l}^{(1)}\right)_{j} & =\left(\bar{\lambda} u_{k, k}^{(1)}\right)_{, i} \\
& =-p_{, i}^{(1)} .
\end{aligned}
$$

Substitute equations (C-20) into equation (C-7), then take its divergence and use equations (A-14) along with (A-15). We recover the constant-density wave equation,

$$
p_{, i i}^{(1)}-\frac{1}{c^{2}} \frac{\partial^{2} p^{(1)}}{\partial t^{2}}=-f_{i, i}^{(1)},
$$

not some other variable-density equation.

The term $\tau_{i j, j}^{(0)}$ in equation $(\mathrm{C}-1)$ achieves relevance in the acoustic setting because of the intimate connection between the background pressure gradient and the density gradient. This link,

$$
p_{, i}^{(0)}=c^{2} \rho_{, i}^{(0)}
$$

was derived in Appendix $\mathrm{A}$ as equation (A-12). Recalling the relation between stress and pressure (equation (C-17)), we see that an elastic solid with a vanishing background stress gradient translates to a constant-density acoustic medium.

However, the background stress gradient, translated into an acoustic situation, places no restriction on the characteristic speed $c$. That is, acoustic speed may vary over space, unaccompanied by a background pressure (density) gradient. In what follows I shall emphasize this principle by writing an acoustic wave equation on displacement, the same dependent variable as in equation (C-1).

Most of the necessary machinery is already in place if one begins with equation (C-18). Equation (A-14) renders $\rho^{(1)}$ in place of $p^{(1)}$, then equation (A-15) replaces $\rho^{(1)}$ with $u^{(1)}$. Following these steps I obtain

$$
\left[c^{2}\left(\rho^{(0)} u_{k}^{(1)}\right)_{, k}\right]_{, i}=c^{2}\left(\rho^{(0)} u_{i}^{(1)}\right)_{, k k}+\left(c^{2}\right)_{, i}\left(\rho^{(0)} u_{k}^{(1)}\right)_{, k}
$$

for the leftmost term of equation (C-18), provided that the displacement field is irrotational. Pooling the substitution results for all terms,

$$
\left(\rho^{(0)} u_{i}^{(1)}\right)_{, k k}+\left[2 \frac{c_{i}}{c}-\frac{\rho_{i}^{(0)}}{\rho^{(0)}}\right]\left(\rho^{(0)} u_{k}^{(1)}\right)_{, k}-\frac{\rho^{(0)}}{c^{2}} \frac{\partial^{2} u_{i}^{(1)}}{\partial t^{2}}=\frac{1}{e^{2}} f_{i}^{(1)} .
$$

A vanishing divergence of the initial stress requires, through exiation (C-22), the vanishing of all density gradients in equation (C-23). Thus, any variabic-density acoustic wave equation hails, of necessity, from an elastic wave equation contaissing the divergence of initial stress. (The divergence of siress is, in its acoustic translation, the gradient of pressure.) 
To the contrary, the speed gradient in equation (C-23) remains a finite quantity, with or without that divergence. This equation allows for spatial variability in $c$, independent of the background-stress gradients in equation (C-1). In circumstances where both speed and density are constant over space, equation (C-23) reduces to the classical wave-equation operator, operating on displacement. 

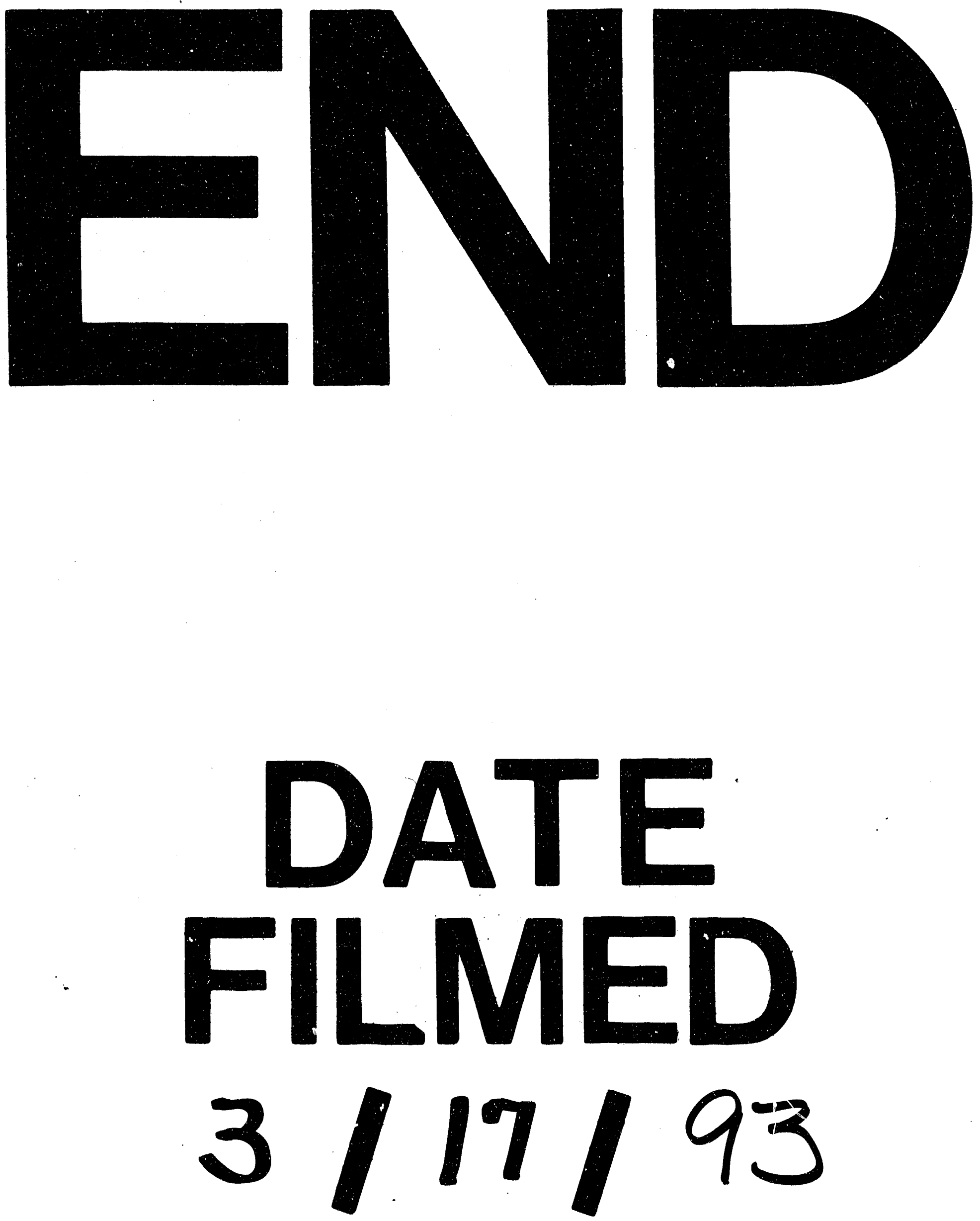
